University of New Hampshire

University of New Hampshire Scholars' Repository

\title{
High-Frequency Broadband Seafloor Backscatter in a Sandy Estuarine Environment
}

Eric J. Bajor

University of New Hampshire - Main Campus

Follow this and additional works at: https://scholars.unh.edu/thesis

\section{Recommended Citation}

Bajor, Eric J., "High-Frequency Broadband Seafloor Backscatter in a Sandy Estuarine Environment" (2015). Master's Theses and Capstones. 5.

https://scholars.unh.edu/thesis/5

This Thesis is brought to you for free and open access by the Student Scholarship at University of New Hampshire Scholars' Repository. It has been accepted for inclusion in Master's Theses and Capstones by an authorized administrator of University of New Hampshire Scholars' Repository. For more information, please contact Scholarly.Communication@unh.edu. 


\section{HIGH-FREQUENCY BROADBAND SEAFLOOR BACKSCATTER IN A SANDY ESTUARINE ENVIRONMENT}

\section{BY}

\section{ERIC J. BAJOR}

B.S. in Mechanical Engineering, University of New Hampshire, 2013

\section{THESIS}

Submitted to the University of New Hampshire

In Partial Fulfillment of

The Requirements for the Degree of

Master of Science

in

Mechanical Engineering

September, 2015 
This thesis has been examined and approved in partial fulfillment of the requirements for the degree of Master of Science in Mechanical Engineering by:

Thesis Director, Thomas C. Weber, Assistant Professor of Mechanical Engineering

Larry A. Mayer, Professor of Earth Sciences

Diane L. Foster, Associate Professor of Mechanical Engineering

On June $1^{\text {st }}, 2015$

Original approval signatures are on file with the University of New Hampshire Graduate School. 


\section{DEDICATION}

I would like to dedicate this thesis to my family and friends for their continued support. Without you, none of this would have been possible. Thank you. 


\section{ACKNOWLEDGEMENTS}

I would like to acknowledge and thank my research advisor, Dr. Thomas C. Weber, for the guidance and insight he has provided me during my M.S. studies. Additionally, I would like to acknowledge the faculty and staff of the University of New Hampshire Center for Coastal and Ocean Mapping and Joint Hydrographic Center (UNH CCOM/JHC). I would also especially like to thank: Paul Lavoie, Maureen Claussen, Carlo Lanzoni, Glen Rice, Larry Mayer, Elizabeth Kintzing, Emily Terry, Ben Smith, Yuri Rhzhanov, Jim Irish, Tom Lippmann, Diane Foster, and Jon Hunt.

I would also like to express my gratitude to my fellow UNH CCOM/JHC students, John Heaton, Meaghan Wengrove, Scott Loranger, Liam Pillsbury, and Han Hu for their help and support throughout this experience. 


\section{TABLE OF CONTENTS}

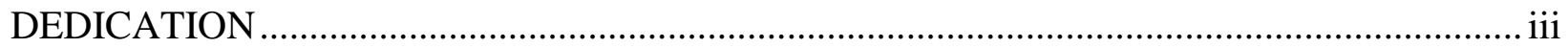

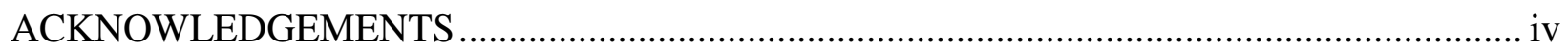

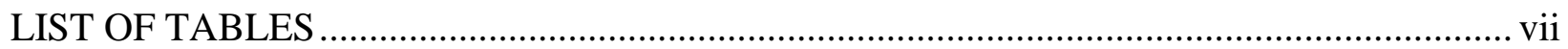

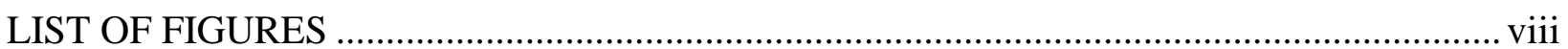

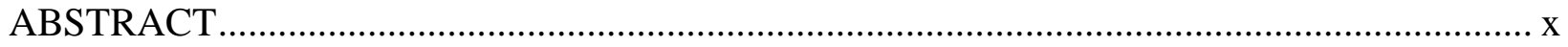

\section{CHAPTER}

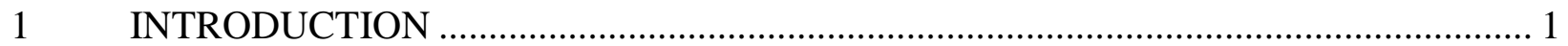

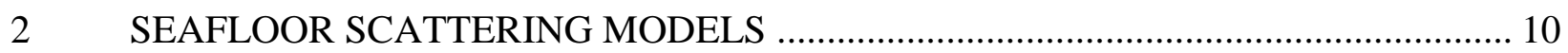

2.1 SURFICIAL ROUGHNESS SCATTERING MODEL ....................................... 10

2.2 DISCRETE INCLUSION SCATTERING MODEL........................................... 14

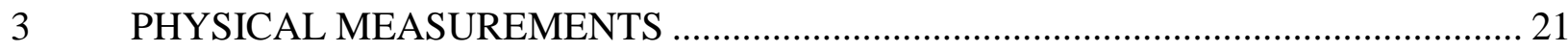

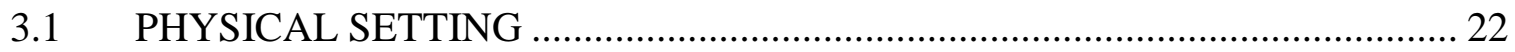

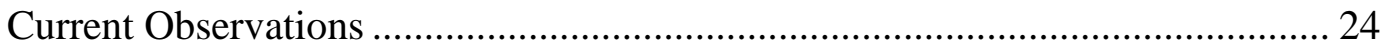

3.2 DATA COLLECTION AND RESULTS ………………………………….... 28

Stereo Imagery Data: Surficial Roughness Scattering ........................................ 28

Sediment Samples: Discrete Inclusion Scattering ........................................... 46

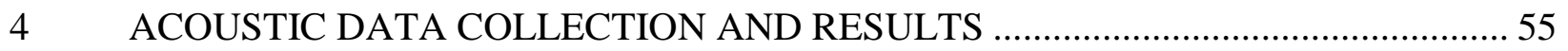

4.1 LOGISTICS OF ACOUSTIC MEASUREMENTS ………………………….... 55

4.2 ACOUSTIC PROCESSING METHODS ………………………………......... 55

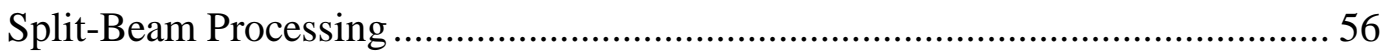


Scattering from an Extended Target: Seafloor Response ............................... 60

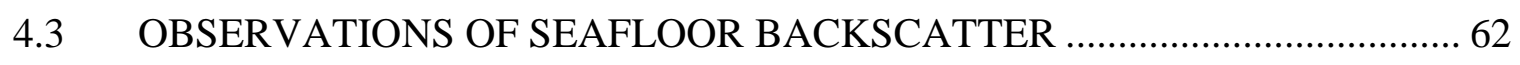

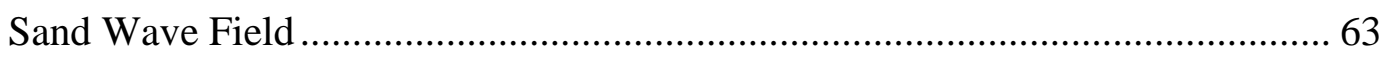

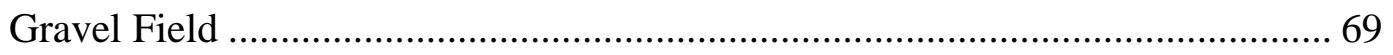

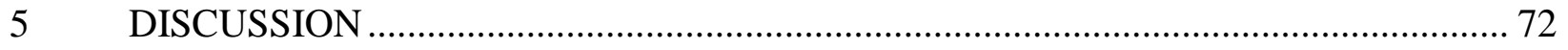

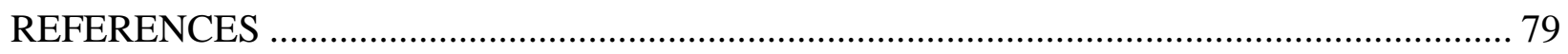

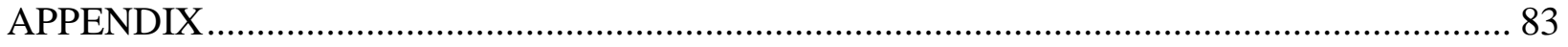




\section{LIST OF TABLES}

Table 2-1. Transition frequency to $k a>1$ for select particle diameter sizes.

Table 3-1. Average slope and corresponding spectral exponent values for roughness spectrum over the sand wave field.

Table 3-2. Average slope and corresponding spectral exponent values for roughness spectrum over the gravel field.

Table 3-3. Grain statistics from Portsmouth Harbor sediment samples utilizing Folk and Ward [1957] logarithmic method. 


\section{LIST OF FIGURES}

Figure 1-1. Illustration of conceptual outline related to the quantification of acoustic backscatter.

Figure 1-2. Experimental location and characterization of major depositional environments

located in Portsmouth Harbor [Ward and McAvoy, 2014] .................................................. 5

Figure 1-3. Relative spatial scale of sedimentary make up of sand wave and gravel fields.......... 7

Figure 2-1. Illustration representing roughness scattering under the assumption that the

transmitted pressure, $p t$ is equal to the sum of the scattered pressure, $p s$ and the incident

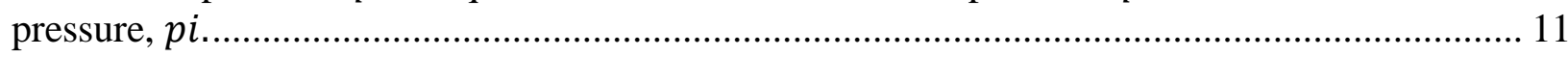

Figure 2-2. Bragg Scattering ....................................................................................... 12

Figure 2-3. Sound penetration depth into seafloor calculated as a function of frequency and a grazing angle of $45^{\circ}$ 16

Figure 2-4. Dimensionless scattering function as a function of $k a$ for particle sizes of $2 \mathrm{~mm}$,

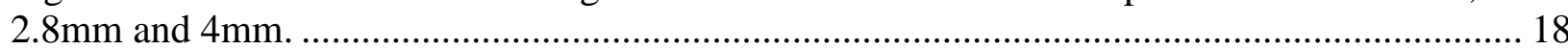

Figure 2-5. Theoretical volume size distribution function and volume scattering coefficient. .... 20

Figure 3-1. Tripod system deployed in the sand wave field containing stereo camera system and

ADCP current profiler (not pictured) ............................................................................. 22

Figure 3-2. Survey transect through sand wave and gravel field............................................. 23

Figure 3-3. Screen grab video data of sand wave and gravel field. ......................................... 24

Figure 3-4. Time series of tide elevation during the 24 hour period surrounding the field

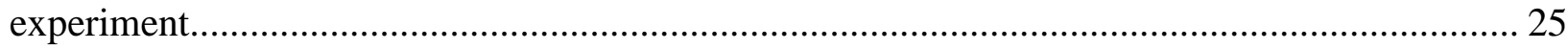

Figure 3-5. Time-series of ADCP current speed in $\mathrm{m} / \mathrm{s}$ and direction is degrees...................... 26

Figure 3-6. Time-series of ADCP current speed (in $\mathrm{cm} / \mathrm{s}$ ) and direction (in degrees) for all bins,

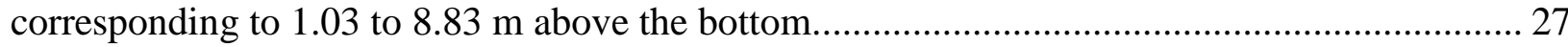

Figure 3-7. Tripod system with mounted stereo camera system. ......................................... 29

Figure 3-8. Close-up view of cameras and LED light panels rigidly mounted to cantilevered arm

attached to the tripod................................................................................................... 29

Figure 3-9. Illustration showing overlap FOV of stereo camera system due to the $10^{\circ}$ tilt of each

camera towards the center of the baseline. ........................................................................ 30

Figure $3-10$. Stereo camera calibration target............................................................ 31

Figure 3-11. Example of uncorrected and corrected calibration images taken from stereo imagery

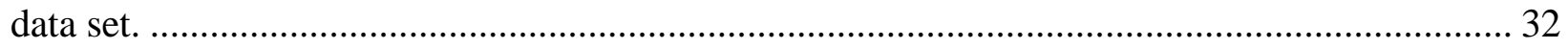

Figure 3-12. Elevation profiles (right) derived from DEM results (left)................................. 34

Figure 3-13. Elevation profiles of large bedform observed by the stereo camera system at the start of the survey (top) and the end of the survey (bottom) ............................................. 35

Figure 3-14. Illustration of example calculation of roughness spectrum for stereo imagery data

collected in the sand wave field. ................................................................................ 36

Figure 3-15. Example roughness spectrum that exhibits behavior of leveling out beyond $225 \mathrm{kHz}$

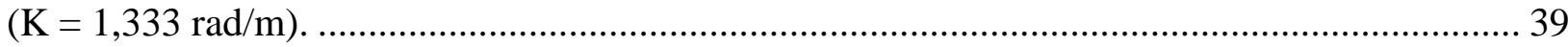

Figure 3-16. Evaluated slopes of roughness spectra for both lower (left) and upper (right)

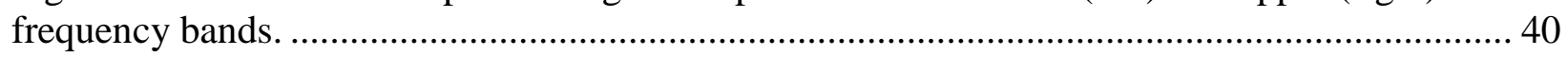

Figure 3-17. Example of two individual spectra calculated from corresponding DEM's. ......... 40

Figure 3-18. Resulting average roughness spectrum over the sand wave field........................ 41

Figure 3-19. Theoretical backscatter prediction for sand wave field produced by roughness scattering model. 
Figure 3-20. DEM example derived from stereo imagery collected in the gravel field. 43

Figure 3-21. Evaluated slopes of roughness spectra for both lower (left) and upper (right)

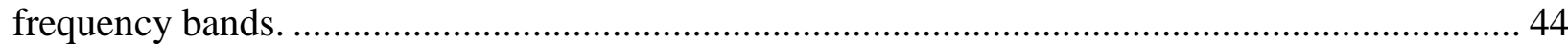

Figure 3-22. Resulting average roughness spectrum over the gravel field............................. 45 Figure 3-23. Theoretical backscatter prediction for gravel field produced by roughness scattering

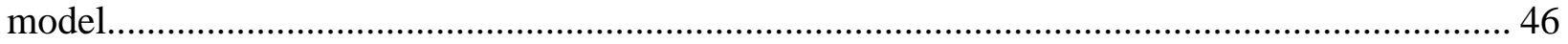

Figure 3-24. Top view illustration of tripod system describing sediment sample collection site. 47 Figure 3-25. Grain size distribution by weight of sediment samples ................................... 49

Figure 3-26. Normalized volume distribution function of sample 1.A................................... 50

Figure 3-27. Inclusion model backscattering results. .................................................. 51

Figure 3-28. Backscatter predictions from inclusion model showing magnitude shift due to variation in sediment acoustic properties, density and sound speed..................................... 52 Figure 3-29. Comparison of predicted scattering strength of inclusion model with variable

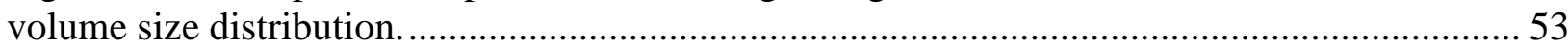
Figure 3-30. Inclusion model scattering backscatter response with line of best fit on upper and

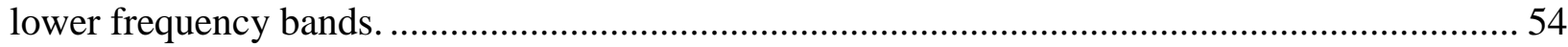
Figure 4-1. Results of $3^{\text {rd }}$ order polynomial fit to $38.1 \mathrm{~mm}$ tungsten carbide calibration sphere (solid black lines).

Figure 4-2. Survey transect through sand wave and gravel field......................................... 62 Figure 4-3. Stability check of acoustic data across the $100 \mathrm{~m}$ transect centered about the tripod.

Figure 4-4. Graphical representation of the bottom detect methods .................................... 65 Figure 4-5. Echogram and across ship phase angle response over a small section of seafloor from

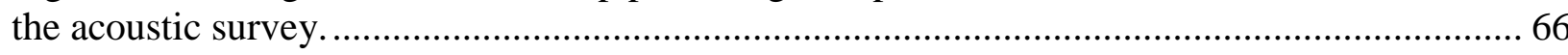
Figure 4-6. Backscatter data as a function of time over the sand wave field showing stability of scattering strength throughout the duration of the survey. .................................................... 67 Figure 4-7. Average bottom backscattering strength over each survey pass as a function of

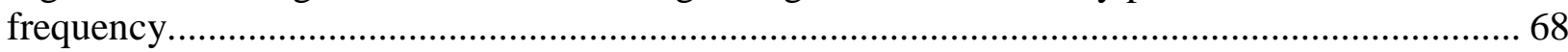
Figure 4-8. Acoustic backscatter results compared to theoretical predictions of backscatter from

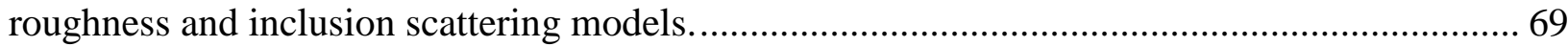
Figure 4-9. Backscatter data as a function of time over the gravel field showing stability of scattering strength throughout the duration of the survey. ................................................. 70 Figure 4-10. Data to model comparison of backscatter observations from the gravel field compared to the roughness scattering model. ............................................................... 71 Figure 5-1. Acoustic backscatter results compared to theoretical predictions of backscatter from roughness and inclusion scattering models................................................................... 75 Figure 5-2. Data to model comparison of backscatter observations from the gravel field compared to the roughness scattering model. .76 


\begin{abstract}
HIGH-FREQUENCY BROADBAND SEAFLOOR BACKSCATTER IN A SANDY ESTUARINE ENVIRONMENT
\end{abstract}

by

Eric J. Bajor

University of New Hampshire, September, 2015

Seafloor backscatter collected with high-frequency (> $100 \mathrm{kHz}$ ) hydrographic echosounders has become an important aspect of seafloor characterization for benthic ecologists and other scientists. The mechanisms that control acoustic scattering at these high frequencies are not completely understood, although surficial roughness and the presence of discrete particles (e.g., shell hash) are likely contributors. To further our understanding of the impact these mechanisms have on seafloor scattering, broadband $(100-250 \mathrm{kHz})$ acoustic measurements were taken at a grazing angle of $45^{\circ}$ in a shallow-water, sandy environment with a known presence of shell hash. Stereo imagery was collected simultaneously to quantify the surficial roughness of the seafloor. Sediment samples were also collected at the site of the experiment to quantitatively analyze the content of shell hash. Backscatter observations between the frequencies of $170 \mathrm{kHz}$ $-250 \mathrm{kHz}$ showed a minimal increase in amplitude with increasing frequency while observations at lower frequencies between $100 \mathrm{kHz}-150 \mathrm{kHz}$ showed an apparent increase in amplitude relative to increasing frequency. Data to model comparisons of the frequency dependence of seafloor backscatter were made to both roughness and discrete particle scattering models indicating neither model is a good descriptor of the seafloor backscatter response. 


\section{CHAPTER 1}

\section{INTRODUCTION}

With over half the world's population living near a coastal region, creating and maintaining accurate maps of the seafloor remains important as overall human impact on the world's oceans is continually increasing [Brown and Blondel, 2009]. One component of such mapping is the collection of acoustic backscatter, which is defined as the amount of acoustic energy received by a sonar after a complex interaction with the seafloor [Stuart, 2012]. The interaction between acoustic waves and the seafloor is complex and driven by irregularities within or at the water-sediment interface including surficial roughness, spatial variations in the sediment physical properties, and by the presence discrete inclusions such as shell pieces, commonly referred to as shell hash [Lurton, 2010].

Backscatter is quantified in terms of the scattering strength of a patch of seafloor ensonified by a sonar's beam as depicted in Figure 1-1. 


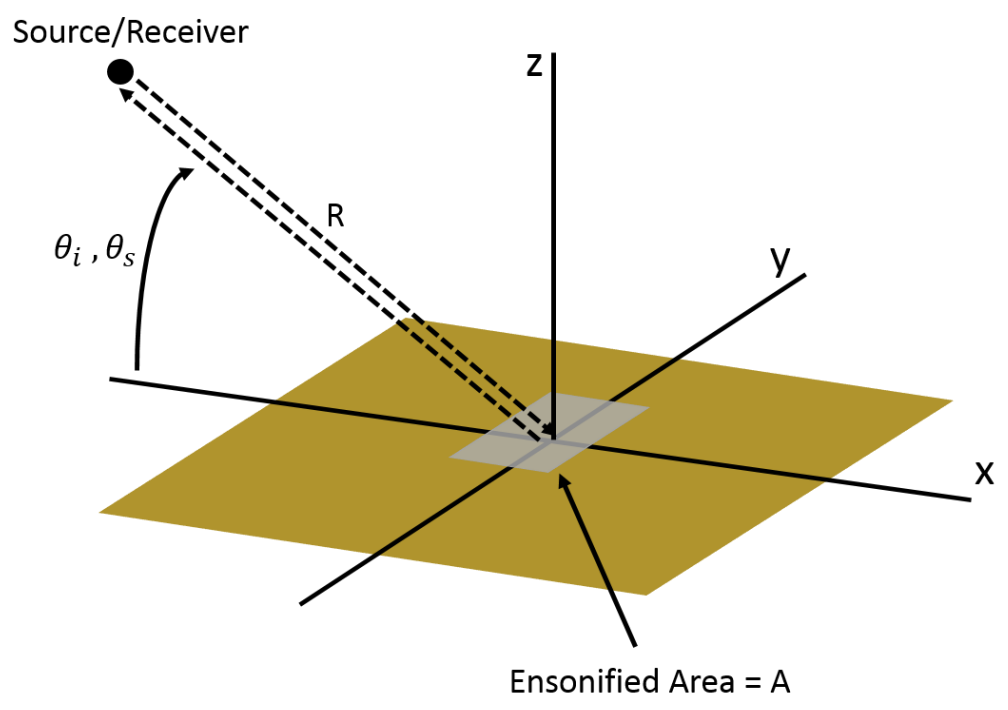

Figure 1-1. Illustration of conceptual outline related to the quantification of acoustic backscatter. $\theta_{i}$ and $\theta_{s}$ are the incident and scattering angles, respectively, and $\mathrm{R}$ is the range from the sonar transducer to the target seafloor.

The scattered pressure field measured by the sonar is defined by Equation 1, in units proportional to intensity, when an ensemble of measurements is taken of statistically equivalent patches of seafloor;

$$
\left\langle\left|P_{S}(R)\right|^{2}\right\rangle=\frac{\left|P_{i}\right|^{2} A \sigma}{R^{2}} e^{-2 \alpha R}
$$

where $P_{S}$ (brackets, \langle\rangle , indicate an ensemble average) is the scattered pressure received by the sonar, $P_{i}$ is the incident pressure at the seafloor, $A$ is the ensonified area, $R$ is the range to the seafloor, $\alpha$ is the absorption coefficient associated with the two-way transmission loss (TL) of the transmitted and received signal, and $\sigma$ is the backscattering cross section [Jackson and Richardson, 2007]. The backscattering cross section is more commonly defined as the "bottom scattering strength' of the seafloor given by the decibel equivalent;

$$
S_{b}=10 \log _{10} \sigma .
$$

Backscatter measurements are collected for their use in seafloor characterization by means of seafloor imaging, seabed mapping, and habitat mapping. Typically, when these 
measurements are collected in less than a few hundred meters water depth, multibeam echosounders (MBES), operated above $100 \mathrm{kHz}$, are utilized [Lurton, 2010]. Researchers and scientists who collect these measurements often use them to create mosaics of seafloor backscatter of a particular survey area [Brown and Blondel, 2009] or to invert the measurements based on theoretical backscatter models to estimate intrinsic properties of the seafloor [Jackson et al, 1996]. However, either use of seafloor backscatter has its own set of challenges. When creating mosaics, data is often combined from multiple surveys in which various MBES may have been used. Changes in backscatter measurements over a single area may then be the result of either the use of varying operating frequencies by different MBES systems or a physical change in seafloor bottom type. Moreover, inversion of backscatter data relies solely on physically based models for acoustic scattering, which are not fully understood at the higher operating frequencies common to MBES systems [Jackson and Richardson, 2007].

Limited modeling done in support of seafloor backscatter at frequencies above $100 \mathrm{kHz}$, makes it unclear to what specific seafloor scattering mechanisms control backscatter measurements at high frequencies. Inconsistencies in backscatter measurements are generally credited to inhomogeneities of a seafloor bottom type, either within the sediment volume, or at the water-sediment interface. The interaction between an acoustic signal and the seafloor will vary based on the presence of inhomogeneities and their size relative to the wavelength of the acoustic signal. For example, it has been suggested that shell fragments overlaying a sandy environment dominate acoustic scattering at high operating frequencies when the size of the shell hash is small relative to the acoustic wavelength [Williams et al., 2002; Ivakin, 2004; Lyons, 2005; Williams et al., 2009]. Similarly, it has also been suggested by models presented by Jackson and Richardson [2007] that seafloor backscatter is dependent on the presence of surficial 
roughness elements that are also small relative to the acoustic wavelength. When such seafloor scattering mechanisms are present within a survey area, the backscatter response from the seafloor is expected to change as the acoustic wavelength, or operating frequency of a sonar, changes with respect to the physical size of seafloor scattering mechanisms related to scattering. A lack of a basic understanding of the mechanisms that control acoustic backscatter at high frequencies limits our ability to properly discriminate between seabed bottom types, ultimately hindering the capability to accurately map the seafloor.

To further understand the relationship between the frequency dependence of acoustic backscatter and seafloor scattering mechanisms discussed, a field experiment was conducted in Portsmouth Harbor, New Hampshire, USA. The harbor is located towards the mouth of the Piscataqua River, and is split by the border between New Hampshire and Maine (Figure 1-2). The bottom composition in the general area of the experiment is diverse, consisting of an assortment of various sediment types including sand, gravel, and bedrock outcroppings. The area is exposed to the ocean and subject to tidally influenced currents. Of particular interest in this study were a sand wave field and gravel field, i.e. channel lag deposits, located in lower Portsmouth Harbor, both of which are shown in Figure 1-2. The sand wave and gravel fields were the chosen areas of interest based on historical data sets that characterized each section of the seafloor prior to experimentation. 


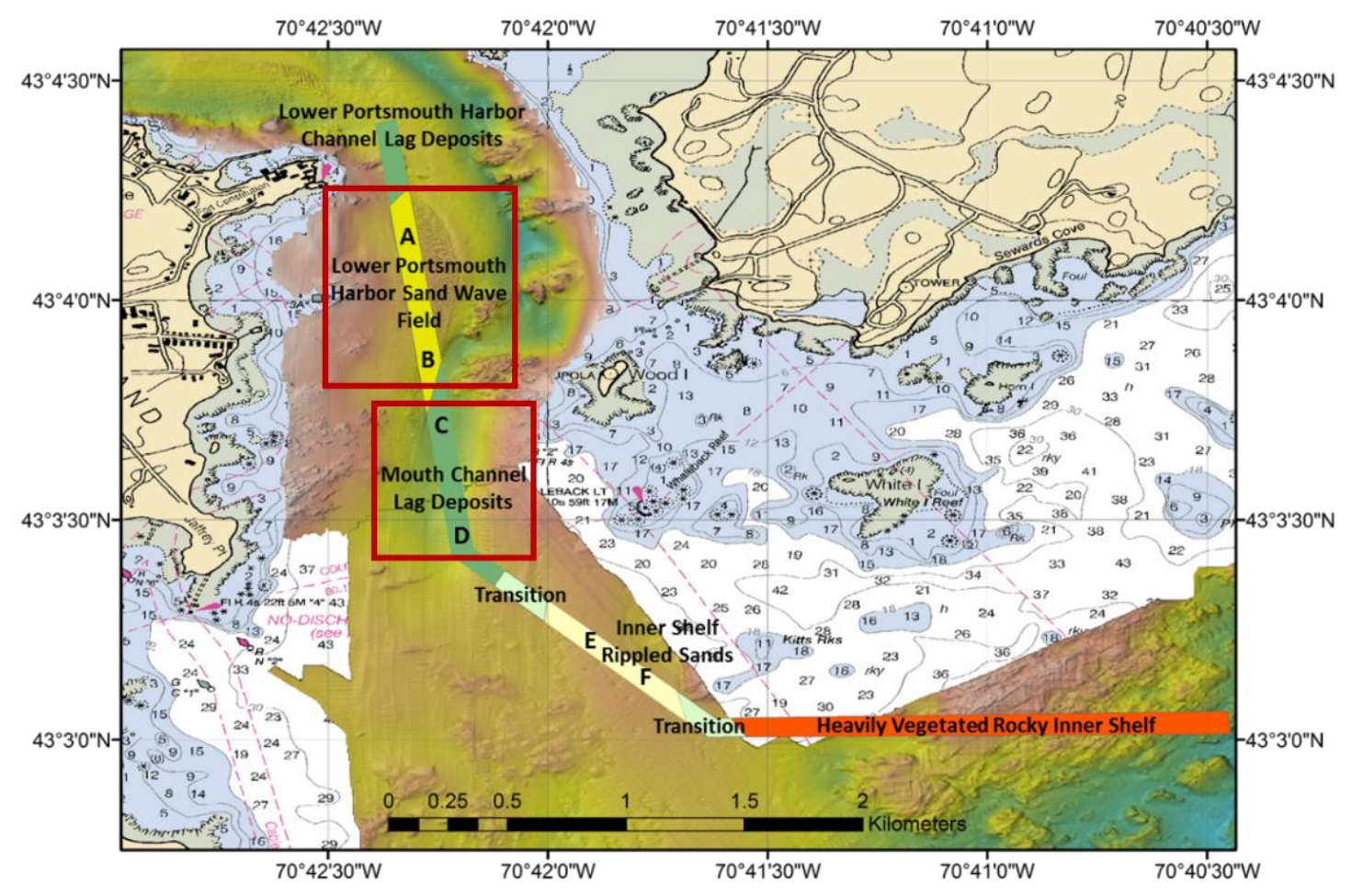

Figure 1-2. Experimental location and characterization of major depositional environments located in Portsmouth Harbor [Ward and McAvoy, 2014]. The experiment described herein took place within the sand wave field and channel lag deposits highlighted by the red boxes.

The sand wave field was characterized by collection of sediment samples [Ward and McAvoy, 2014], video screen grabs [Felzenburg, 2009; Ward and McAvoy, 2014], current observations [Felzenburg, 2009], and multiple MBES surveys [Felzenburg, 2009]. MBES surveys were conducted between June 2007 and July 2008 and revealed the presence of large sand dunes ranging from approximately 3 to $5 \mathrm{~m}$ in wavelength and 0.1 to $0.5 \mathrm{~m}$ in height on the eastern periphery of the sand wave field. In addition to the large sand dunes, video screen grab data revealed the presence of smaller sand ripples approximately $\sim 0.20 \mathrm{~m}$ in wavelength overlaying the sand dunes. The combination of sediment samples and current observations taken in the sand wave field by Felzenburg [2009] indicated that current magnitude during both flood and ebb tide events exceeded the critical threshold for incipient motion of the sediment based on 
mean grain size of the sediment, inferring a change in the surficial roughness of the sand wave field with respect to time. However, grain size distribution statistics of the sediment remained relatively uniform in time. This was confirmed by samples collected by Ward and McAvoy [2014], as compared to samples collected by Felzenburg [2009], following multiple sediment sample and video data cruises occurring on June $20^{\text {th }}$, October $21^{\text {st }}$, and December $17^{\text {th }}$ in 2013. Consistency of the sediment samples suggests spatial stability within the sand wave field with respect to time despite the dynamic nature of the area. Inspection of samples taken by Ward and McAvoy [2014] also revealed the presence of high shell hash content located in the sand wave field. Shell hash grain size ranged from $0.5 \mathrm{~mm}$ to $4 \mathrm{~mm}$ and accounted for nearly $20 \%$ of sediment samples.

The gravel field was also characterized by grain size from sediment samples and video data collected during the sampling cruises in 2013. Overall mean grain size distribution of the gravel field ranged from $4 \mathrm{~mm}$ to $33 \mathrm{~mm}$ with a mean grain size of approximately $\sim 12 \mathrm{~mm}$ [Ward and McAvoy, 2014].

Historical characterization of the sand wave and gravel fields provided adequate environmental description of each area needed to design an experiment aimed at providing insight to the linkages between the seafloor scattering mechanisms inherent to both areas (i.e. surficial roughness, presence of discrete inclusions) and acoustic backscatter measurements. The main portion of the experiment involved a broadband acoustic survey during the dynamic period of a flood tide event. Backscatter observations were collected between $90 \mathrm{kHz}-260 \mathrm{kHz}$ utilizing two split-beam echosounders (SBES), to examine the frequency and temporal dependence of backscatter observations over both sand wave and gravel fields. The frequency response of the measurements was limited to upper and lower frequency bands of $170 \mathrm{kHz}-250$ 
$\mathrm{kHz}$ and $100 \mathrm{kHz}-150 \mathrm{kHz}$, respectively. This limitation was due to the distorted nature of the broadband signals produced by each individual SBES used during the survey.

The operating frequencies used during the experiment corresponded to the small scales of the seafloor scattering mechanisms of both the sand wave and gravel fields as determined by Felzenburg [2009] and Ward and McAvoy [2014]. The acoustic operating frequency range covered a span of acoustic wavelengths from $6 \mathrm{~mm}-15 \mathrm{~mm}$ within an estimated footprint on the seabed of approximately $3.5 \mathrm{~m}^{2}$ over the sand wave field and $5.5 \mathrm{~m}^{2}$ over the gravel field. Increased area of the sonar's beam on the seabed was due to an increase in depth of the water at the gravel field location. The scales of seafloor scattering mechanisms found in the sand wave and gravel fields pertinent to the acoustic backscatter observations characterized by spatial scale are shown in Figure 1-3.

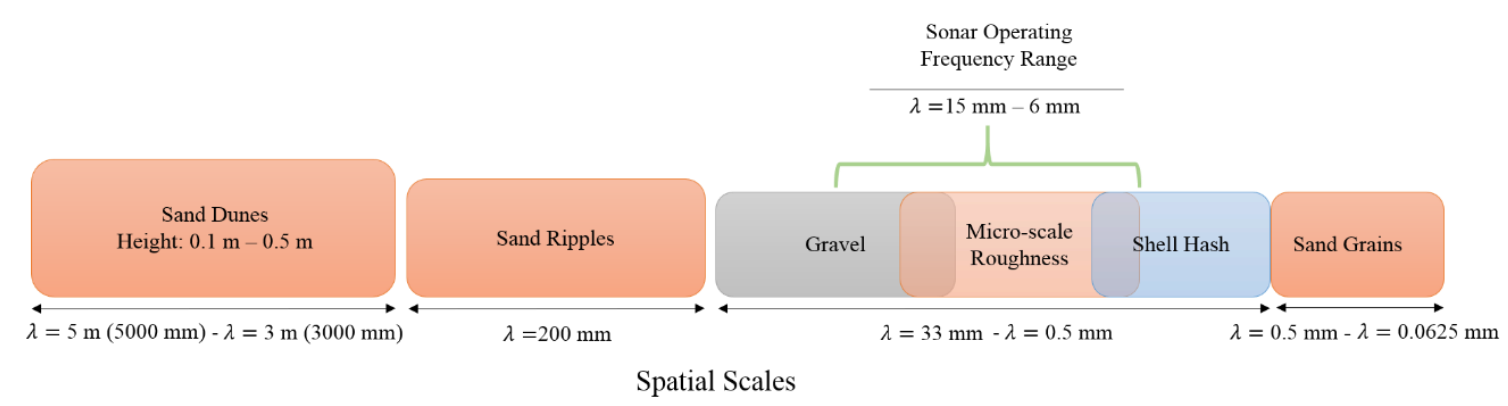

Figure 1-3. Relative spatial scale of sedimentary make up of sand wave and gravel fields. Acoustic wavelength values on the $\mathrm{x}$-axis were determined by historical seafloor characterization of the sand wave and gravel fields by Felzenburg [2009] and Ward and McAvoy [2014].

Both sites contained seafloor scattering mechanisms characterized as surficial roughness features, provided by the pebbly nature of the gravel field and micro-scale sand ripples in the sand wave field. The sand wave field was also characterized as an area containing discrete inclusions, by the presence of shell hash in a predominantly sandy area. 
Stereo imagery data sets were also collected as part of the field experiment in both sand wave and gravel fields to quantitatively describe the surficial roughness of each area.

Quantitative description of the surficial roughness was desired as input to a roughness scattering model [Jackson and Richardson, 2007] to make comparisons between theoretical predictions of backscatter and acoustic backscatter observations. Stereo imagery was collected in the sand wave field simultaneous to the collection of acoustic backscatter. This was done due to the dynamic nature of the sediment controlled by the tidally induced currents. Predicted motion of the sediment bottom created the hypothesis that the surficial roughness of the sand wave field was changing in time, which would ultimately influence the acoustic backscatter measurements, if they were indeed controlled by surficial roughness.

Stereo imagery was collected in the gravel field following the acoustic survey under the assumption that the gravel remained stationary in time due to the larger grain size distribution of the sediment ( $4 \mathrm{~mm}-33 \mathrm{~mm}$ grain sizes). Historical consistency of sediment samples collected by Ward and McAvoy [2014] in the gravel field added confidence to the assumption that the gravel field was spatially stable in time when theoretical predictions of backscatter were compared to acoustic observations.

A final data set of sediment samples from the sand wave field was also collected in part for the field experiment to provide input to a discrete inclusion scattering model [Ivakin, 2004] utilized to compare a theoretical prediction of backscatter based on the presence of discrete inclusions (i.e. shell hash) to the acoustic backscatter observations. Input needed from the sediment samples was focused around the grain size distribution of the sediment. Sediment samples were collected by divers in situ just prior to the acoustic survey and stereo camera data set collection. 
The main objective of the experiment described was to identify the seafloor scattering mechanism that was the main contributor to acoustic backscatter in the sand wave and gravel fields. This was done by directly relating the frequency dependence of the observed acoustic backscatter to the frequency dependence of theoretical backscatter predictions generated from surficial roughness and discrete inclusion scattering models. Each model assumed the frequency response of backscatter was controlled by the respective seafloor scattering mechanism of interest. An accurate comparison of either model to the acoustic backscatter would suggest surficial roughness or the presence of discrete inclusions as the controlling mechanism of the backscatter response depending on the model being compared. Input parameters from the seafloor environment necessary for both scattering models were derived from the stereo imagery data sets with respect to surficial roughness and the sediment samples with respect to the presence of discrete inclusions. 


\section{CHAPTER 2}

\section{SEAFLOOR SCATTERING MODELS}

Both roughness scattering and inclusion scattering models were used to compare empirically observed acoustic backscatter to theoretical predictions of backscatter derived from each model. The frequency response of the theoretical predictions was the main focus of the comparisons and not the corresponding amplitude of the results, under the assumption that it would best indicate the underlying seafloor scattering mechanism (i.e. surficial roughness, discrete inclusions) controlling the acoustic backscatter response. Model predictions were adjusted in amplitude and overlaid on the acoustic results for direct comparison of the frequency dependence. Input parameters for each model were obtained from the stereo imagery and sediment sample data sets. For either model, the assumption was made that the controlling mechanism of the backscatter response was either surficial roughness or the presence of discrete inclusions for the respective model of interest.

\subsection{Surficial Roughness Scattering Model}

In the present work the roughness scattering model utilized in comparing the theoretical frequency response of backscatter to the observed frequency response of the acoustic backscatter measurements is based on small-roughness perturbation approximation theory. Small-roughness perturbation approximation theory analyzes the scattering from a randomly rough surface with excursions that are small compared to the acoustic wavelength of the operating sonar transducer. The analysis is made under the boundary condition that the transmitted pressure from an acoustic 
signal is equal to the sum of the scattered and incident pressures, shown in Figure 2-1 [Weber, 2014].

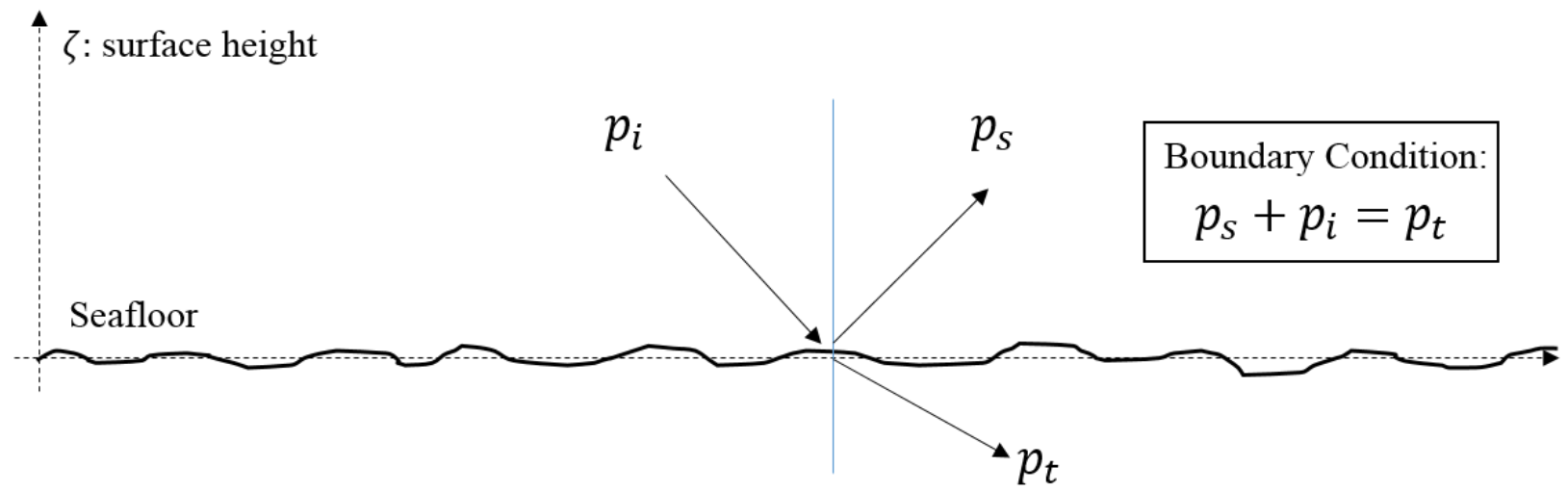

Figure 2-1. Illustration representing roughness scattering under the assumption that the transmitted pressure, $p_{t}$ is equal to the sum of the scattered pressure, $p_{s}$ and the incident pressure, $p_{i}$.

Expansion and evaluation of the boundary condition in a Taylor series around the average surface height (e.g. $\zeta=0$ ), incorporating only the 'zeroth' and first order terms of the Taylor series, representing the smooth surface reflection coefficient and roughness elements, respectively. The first order terms of the Taylor series expansion, representing the roughness elements, are proportional to $k_{w} \zeta$, where $k_{w}$ is the acoustic wave number defined by,

$$
k_{w}=\frac{2 \pi}{\lambda}
$$

Equation 3

where $\lambda$ is the acoustic wavelength. Under the assumption that the random seafloor surface heights projected onto the direction of the acoustic wave are much less than one, $k_{w} \zeta$ is considered small. Terms beyond the first order can then be considered negligibly small and ignored from the Taylor series approximation [Weber, 2014]. This analysis results in a backscattering cross section proportional to the 2D roughness spectrum, $W(\Delta K)$, evaluated at the Bragg wave vector, $\Delta K$ [Jackson and Richardson, 2007]; 


$$
\sigma=k_{w}^{4}|A|^{2} W(\Delta K)
$$

Equation 4

where $\mathrm{A}$ is a constant.

The Bragg wave vector is defined as the difference between the horizontal components of the incident wave vector $K_{i}$, produced by a sonar transducer, and the scattered wave vector $K_{s}$. If the roughness spectrum of the seafloor has a large peak at some wave vector $K_{0}$, there will be strong scattering in two directions corresponding to $K_{s}=K_{i} \pm K_{0}$, known as Bragg scattering [Jackson and Richardson, 2007]. Bragg scattering assumes that for a given angle of incidence $\theta_{i}$, the backscatter response is dominated by contributions from scatterers whose return signals are in phase as seen in Figure 2-2 [Lurton, 2010]. These scatterers, resolved as the roughness spectrum of the seafloor, are distributed at a distance $d$ from each other with $2 d \cos \theta_{i}=n \lambda$ for $=1,2, \ldots[$ Lurton 2010].

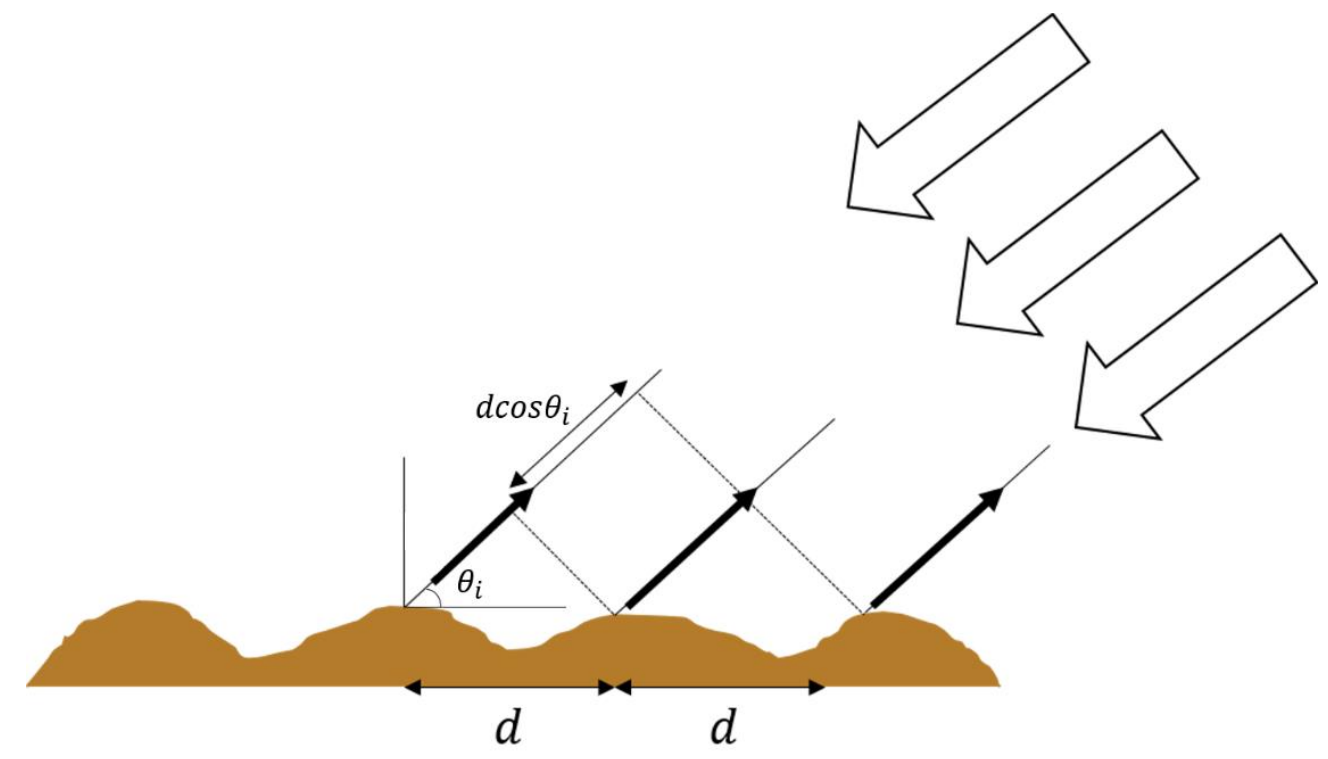

Figure 2-2. Bragg Scattering: at incidence angle $\theta_{i}$, the main contribution to backscatter comes from scatterers with spacing $d$ such as $2 d \sin \theta_{i}=\lambda$, building a constructive interference pattern. [Lurton, 2010]

This condition is satisfied at all angles when the seafloor is isotropic and the Bragg wavenumber is defined by, 


$$
\Delta K=2 k_{w} \cos \theta_{i}
$$

Equation 5

where $\theta_{i}$ is the grazing angle. The Bragg wavenumber relates the physical size of the acoustic wavelength to the size of the roughness elements present on seafloor. For this study, relevant Bragg wavenumber quantities ranged in magnitude from $\sim 4.5 \mathrm{~mm}$ to $\sim 6 \mathrm{~mm}$ for the upper band of acoustic survey frequencies and $\sim 7 \mathrm{~mm}$ to $10.5 \mathrm{~mm}$ for the lower band of acoustic survey frequencies.

The roughness spectrum of the seafloor can be resolved by applying the 2D Fourier transform (2D-FFT) to digital elevation models (DEM) or bathymetry maps obtained from a stereo imagery data set. The 2D-FFT is applied to a DEM by Equation 6;

$$
W\left(k_{x}, k_{y}\right)=\iint_{-\infty}^{\infty} w(x, y) e^{-j 2 \pi\left(k_{x} x+k_{y} y\right)} d x d y, \quad \text { Equation } 6
$$

where $k_{x}$ and $k_{y}$ are wave vectors in $x$ and $y$ directions, respectively, and $W\left(K_{x}, K_{y}\right)$ is the 2D spectrum of the DEM matrix, $w(x, y)$.

The $2 \mathrm{D}$ roughness spectrum can be characterized by the slope and intercept of the linear regression line through a 'slice' of the 2D spectrum evaluated at the Bragg wavenumber, estimated in log-log space [Briggs et al., 2005]. A slice taken from the 2D-FFT equates to the $1 \mathrm{D}$ spectrum obtained by integrating the $2 \mathrm{D}$ spectrum over the wave vector component orthogonal to the 1D measurement track [Jackson and Richardson, 2007]. This relationship is described by the following;

$$
W_{1}\left(K_{x}\right)=\int W_{2}\left(K_{x}, K_{y}\right) d K_{y}
$$

where subscripts 1 and 2 indicate the number of dimensions of the spectrum. $K$ is the Bragg wavenumber in respective subscript directions, $x$ and $y$. 
Characterization utilizing 1D slices through the 2D roughness spectrum is made under the assumption that the roughness spectrum obeys a simple power law,

$$
W_{2}(K)=\frac{\omega_{2}}{K^{\gamma_{2}}} \quad \text { Equation } 8
$$

where $\omega_{2}$ is the spectral strength related to the intercept of the linear regression line of the spectrum slice and $\gamma_{2}$, the spectral exponent, is the absolute value of the spectral slope plus unity [Jackson et al. 1996]. The magnitude of the overall spectrum evaluated at a given value of the Bragg wavenumber describes the amount of acoustic energy returned from a seafloor scattering mechanism whose physical size relates to the corresponding Bragg wavenumber. Significant to this study, the spectral exponent relates to the frequency response of acoustic backscatter in terms of small-roughness perturbation approximations by the relationship,

$$
\sigma(f)=\text { constant } f^{4-\gamma_{2}} \quad \text { Equation } 9
$$

where $\sigma$ is the backscattering cross section, and $f$ is frequency [Jackson and Richardson, 2007]. Typically, most seafloors possess a roughness spectrum on the order of $K^{-3}$ to $K^{-3.5}$ resulting in an increase in backscattering strength of $\sim 3 \mathrm{~dB}$ when doubling the operating frequency of a sonar transducer [Weber, 2014].

\subsection{Discrete Inclusion Scattering Model}

An inclusion scattering model developed by Ivakin [2004] was also utilized in comparing the frequency dependence of acoustic backscatter observations to theoretical predictions of backscatter under the assumption that the presence of discrete inclusions was mechanism controlling the backscatter response of the seafloor. Similar to the comparison of the roughness scattering model to backscatter observations, the objective of the comparison was to directly compare the frequency response of the theoretical prediction to the frequency response of the acoustic observations under the assumption that the analysis of the frequency response would 
best indicate the underlying seafloor scattering mechanism (i.e. surficial roughness, discrete inclusions) controlling the acoustic backscatter response.

The inclusion model assumes that the presence of discrete inclusions or scatterers, such as shell hash or coarse sand grains, that are large compared to the mean grain size of a sample distribution, are the controlling mechanisms of the backscatter response. Theoretical backscatter predictions are described in terms of the individual scattering functions of discrete targets and statistical distributions of parameters, such as size, shape, and material makeup [Ivakin, 2004].

The inclusion model assumes that seabed scattering is due to volume heterogeneity of the sediment with average acoustic parameters of sound speed and density. Sound speed and density of individual particles and the surrounding sediment volume are assumed to be independent of depth [Ivakin, 2004]. A seabed backscattering coefficient, or backscattering cross section per unit area of the seabed surface, which describes a theoretical section of seafloor comprised of specific sediment parameters that contributes to the backscatter response, can be estimated as,

$$
m_{s}=|W|^{4} \mu^{-2} m_{v} h_{p}\left(f, \mathrm{X}_{\mathrm{w}}\right), \quad \text { Equation } 10
$$

where $W$ is the sound transmission coefficient of the water-sediment interface, $\mu$ is the sediment to water density ratio, $h_{p}$ is the sound penetration depth, and $m_{v}$ is the volume backscattering coefficient of the sediment. For purposes of this analysis, the sound transmission coefficient and water to sediment density ratio were neglected in this calculation. Both parameters are constants and only effect the overall magnitude of the resultant theoretical prediction of backscatter based on the model and not the frequency response. Equation 10 was then simplified to,

$$
m_{s}=m_{v} h_{p}\left(f, \mathrm{X}_{\mathrm{w}}\right), \quad \text { Equation } 11
$$

becoming a function of only the sound penetration depth and the volume scattering coefficient. 
The depth of sound penetration into the sediment is calculated as a function of frequency, $f$ and grazing angle, $\mathrm{X}_{w}$;

$$
h_{p}=\frac{\operatorname{Re} \sqrt{\left(n^{2}-\cos ^{2} \mathrm{X}_{w}\right)}}{2 \beta n_{o}}
$$

Equation 12

where $\beta=2 k \delta, k=\frac{2 \pi f}{c_{w}}$, and $n=n_{o}(1+i \delta)$ are the attenuation coefficient, wavenumber, and complex refraction index of the sediment, respectively. $\delta$ is the loss parameter, and $n_{o}$ is the water to sediment sound speed ratio $\left(\frac{c_{w}}{c}\right)$. Loss parameter and sediment sound speed parameters were estimated for calculation of the sound penetration depth as 0.01 and $1700 \mathrm{~m} / \mathrm{s}$, respectively [Ivakin, 2004].

Sound penetration depth estimates decrease with increasing frequency as depicted in Figure 2-3. Maximum penetration depths are seen at the lowest frequencies and decrease as frequency increases.

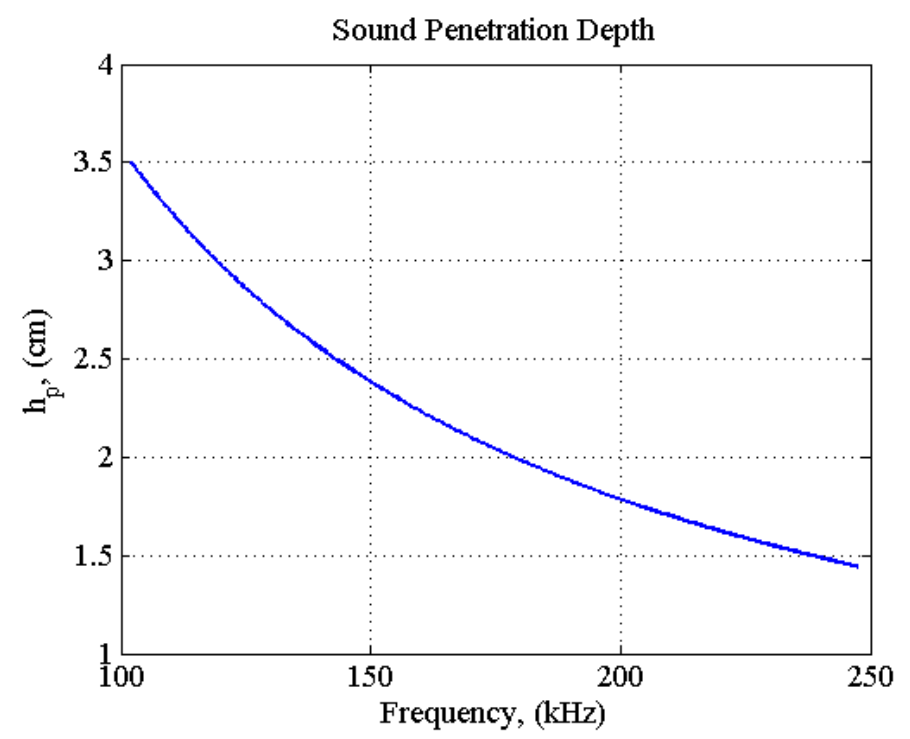

Figure 2-3. Sound penetration depth into seafloor calculated as a function of frequency and a grazing angle of $45^{\circ}$.

The volume scattering coefficient, $m_{v}$, is estimated as, 


$$
m_{v}=\frac{3}{4 \pi} \int F(k a) a^{-2} \psi_{v}(a) d a
$$

Equation 13

where $F$ is a dimensionless scattering function, $\psi_{v}$ is a volume size distribution function, and $a$ is the equivalent radius of individual scatterers (radius of a sphere having the same volume as a non-spherical inclusion). Variable changes in magnitude of the volume scattering function with respect to frequency and particle grain size are controlled by the response of both the dimensionless scattering function and the overall grain size distribution of the sediment sample, which is directly correlated to the volume size distribution function. The volume size distribution function is calculated by,

$$
\psi_{v}=\frac{4}{3} \pi a^{4} \psi_{N}(a)
$$

Equation 14

where the number size distribution function, $\psi_{N}(a)$ is given by,

$$
\psi_{N}(a)=\frac{\Delta N_{a}}{V \Delta a} .
$$

where $\Delta N_{a}$ is the total number of particles in a sieve interval, and $V$ is the entire sediment sample volume. The dimensionless scattering function, $F$ is defined as,

$$
F(k a)=\frac{R_{o}^{2}}{4}(k a)^{4}\left(1+\frac{R_{o}^{2}}{R^{2}}(k a)^{4}\right)^{-1},
$$

where,

Equation 16

$$
R_{o}=\frac{2}{3}\left(1-\frac{\rho c^{2}}{\rho_{\alpha} c_{\alpha}^{2}}\right)+\frac{\rho_{\alpha}-\rho}{\rho_{\alpha}+\rho / 2}, R=\frac{\rho_{\alpha} c_{\alpha}-\rho c}{\rho_{\alpha} c_{\alpha}+\rho c} .
$$

Density $(\rho)$, and sound speed (c) define the sediment properties in terms of individual particles with the subscript $\alpha$ and the surrounding sediment as $\rho$ and $c$. Analysis of the dimensionless scattering function predicts Rayleigh scattering at frequencies that correspond to a value of $k a \ll$ 1 for individual particle sizes. Rayleigh scattering occurs from particles that are much smaller 
than the acoustic wavelength. In such a case, omni-directional scattering is assumed and the shape of the particle is irrelevant. However, in the regime where $k a>1$, where particle size is not small compared to the acoustic wavelength, the assumption is made that directional scattering occurs and the shape of the particle directly effects the scattering. Figure 2-4 depicts this relationship for particles with diameters of $2 \mathrm{~mm}, 2.8 \mathrm{~mm}$ and $4 \mathrm{~mm}$. Transition frequencies for each particle size from non-directional to directional scattering are found in Table 2-1.

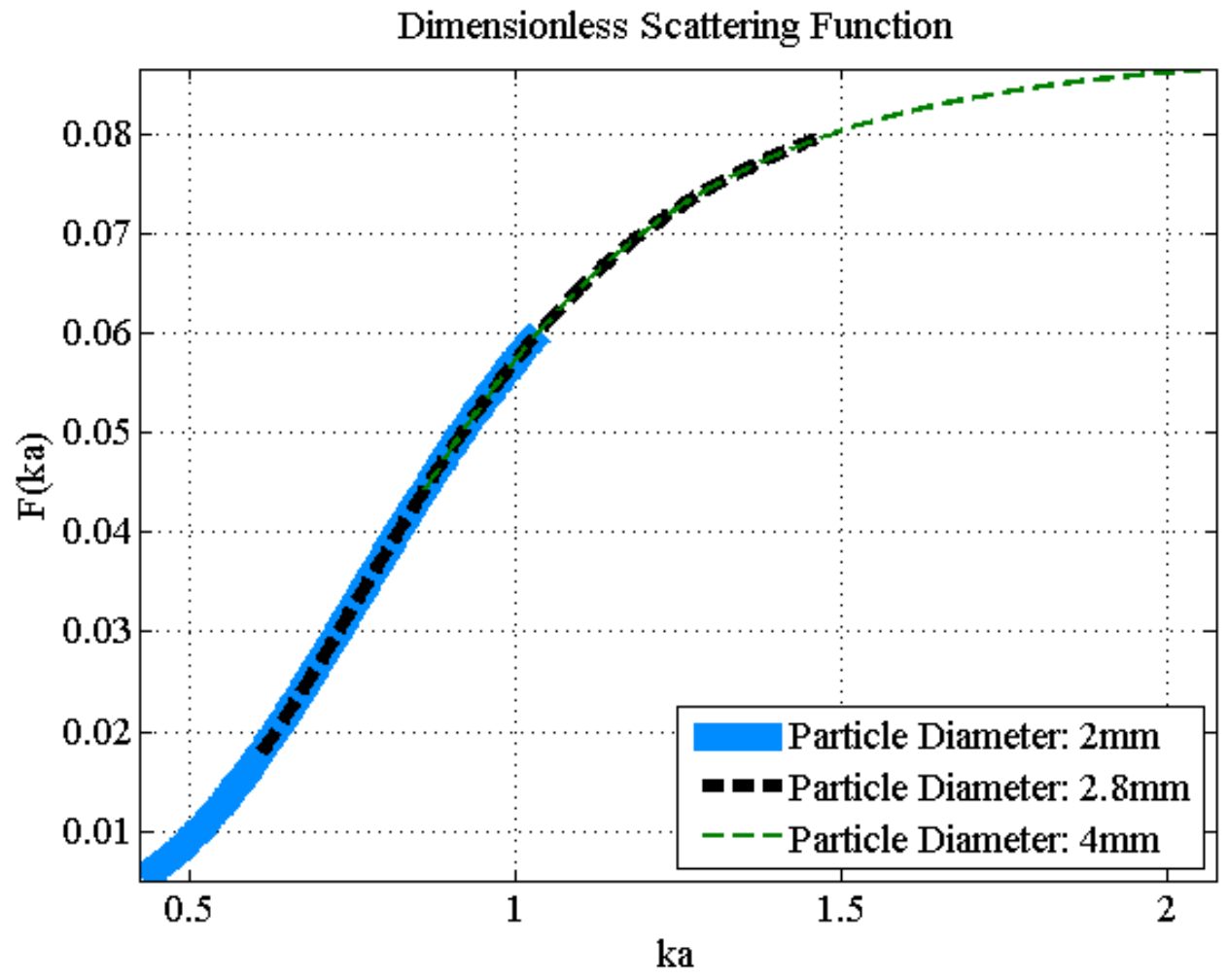

Figure 2-4. Dimensionless scattering function as a function of $k a$ for particle sizes of $2 \mathrm{~mm}, 2.8 \mathrm{~mm}$ and $4 \mathrm{~mm}$.

Table 2-1. Transition frequency to $k a>1$ for select particle diameter sizes.

\begin{tabular}{|c|c|}
\hline Particle Diameter, $\mathrm{mm}$ & Transition Frequency, $\mathrm{ka}>1$ \\
\hline 2 & 238.5 \\
\hline 2.8 & 168.5 \\
\hline 4 & 119 \\
\hline
\end{tabular}


Results of the dimensionless scattering function for all other particle sizes with a diameter less than $2 \mathrm{~mm}$ remains in the region where $k a \ll 1$.

Behavior of the dimensionless scattering function that describes the difference between Rayleigh scattering and non-Rayleigh scattering of discrete inclusions is important to note in conjunction with the volume size distribution function related to the volume scattering coefficient. Although the dimensionless scattering function will remain constant regardless of the sediment sample distribution, given it is only a function of sediment size and frequency, its impact on the magnitude of the volume scattering coefficient will vary based on the volume size distribution function. For example, particles with large diameters extending beyond the limits of Rayleigh scattering will have more of an impact on the volume scattering function based on the higher magnitude of the dimensionless scattering function in comparison to smaller sized particles. However, if only few particles of these larger diameters are present within the sample, their impact on the volume scattering function will be reduced due to the low magnitude of the volume size distribution function. Evidence of this behavior can be seen in Figure 2-5 for acoustic operating frequencies of $120 \mathrm{kHz}$ and $200 \mathrm{kHz}$ given a theoretical grain size distribution, also shown in Figure 2-5. Generally, larger grain sizes have an increased effect on the magnitude of the volume scattering coefficient. However, the volume scattering coefficient decreases as a function of grain size beginning at approximately $3.5 \mathrm{~mm}-4 \mathrm{~mm}$ when the acoustic operating frequency is equal to $120 \mathrm{kHz}$. This is due to the decrease in the volume distribution function at larger grain sizes outweighing the increase in the dimensionless scattering function, which limits the effect of the dimensionless scattering function on the volume scattering coefficient. 

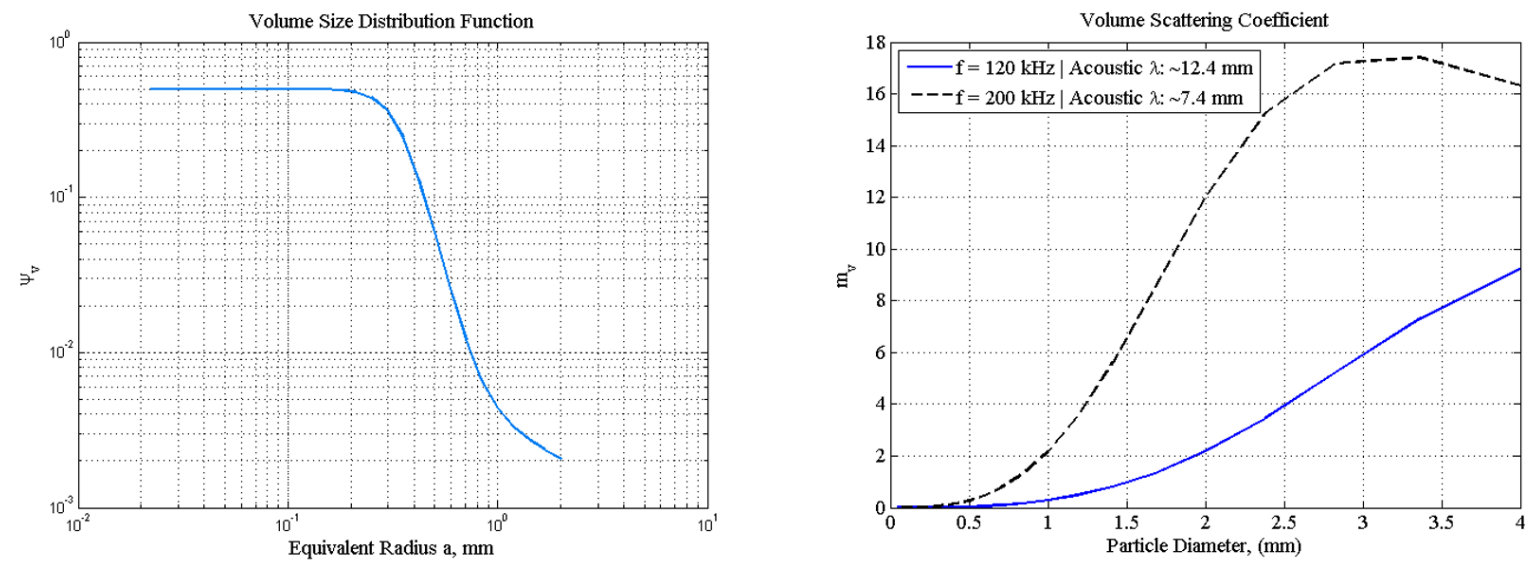

Figure 2-5. Theoretical volume size distribution function and volume scattering coefficient.

As a result, theoretical predictions of backscatter are determined by conversion of Equation 10 to its decibel equivalent;

$$
S_{b}=10 \log _{10} m_{s} .
$$

Equation 17 


\section{CHAPTER 3}

\section{PHYSICAL MEASUREMENTS}

Sediment samples, acoustic Doppler current profiler (ADCP) observations, underwater stereo photography, and SBES backscatter observation data sets were acquired to evaluate the frequency dependence of seafloor backscatter strength, and the mechanisms believed to control this dependence, within the sand wave field in Portsmouth Harbor on 1 October 2014. With the exception of the sediment samples, data related to the sand wave field was collected during the high magnitude current of a flood tide to capture the dynamic current driven properties of the seafloor. Sediment samples within the sand wave field were collected by divers at slack tide prior to flood tide, for diver safety. The ADCP and stereo photography data sets were collected via a tripod system (Figure 3-1) deployed on site by divers. Acoustic observations of the gravel field were also collected during the survey period. Stereo imagery related to the gravel field was collected after the survey period upon retrieval of the system from the sand wave field. 


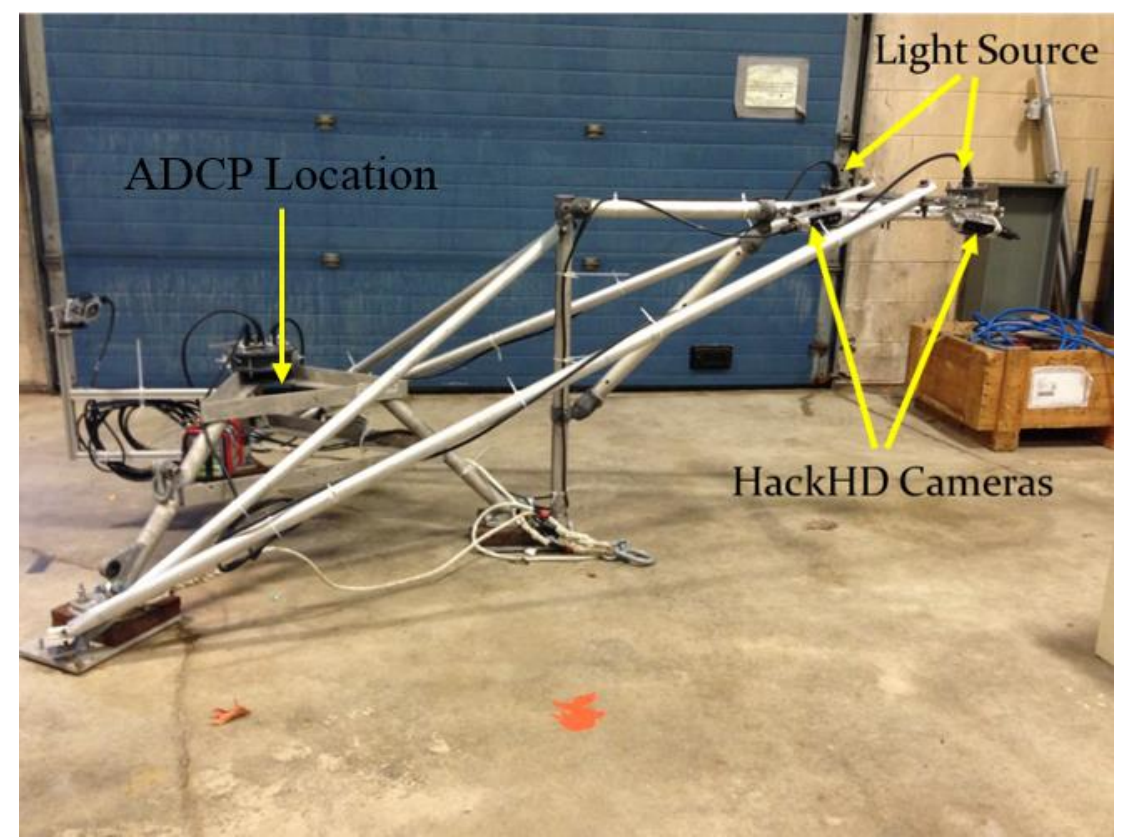

Figure 3-1. Tripod system deployed in the sand wave field containing stereo camera system and ADCP current profiler (not pictured).

\subsection{Physical Setting}

The survey line selected for acoustic data collection, passed over both sand wave and gravel fields located in Portsmouth Harbor. Seafloor bedforms and sediment grain sizes in both areas were relevant to acoustic operating frequencies between $100 \mathrm{kHz}-250 \mathrm{kHz}$ corresponding to physical acoustic wavelengths of $6 \mathrm{~mm}$ to $15 \mathrm{~mm}$. The transect was approximately $600 \mathrm{~m}$ long, and is outlined by the orange line in Figure 3-2. 


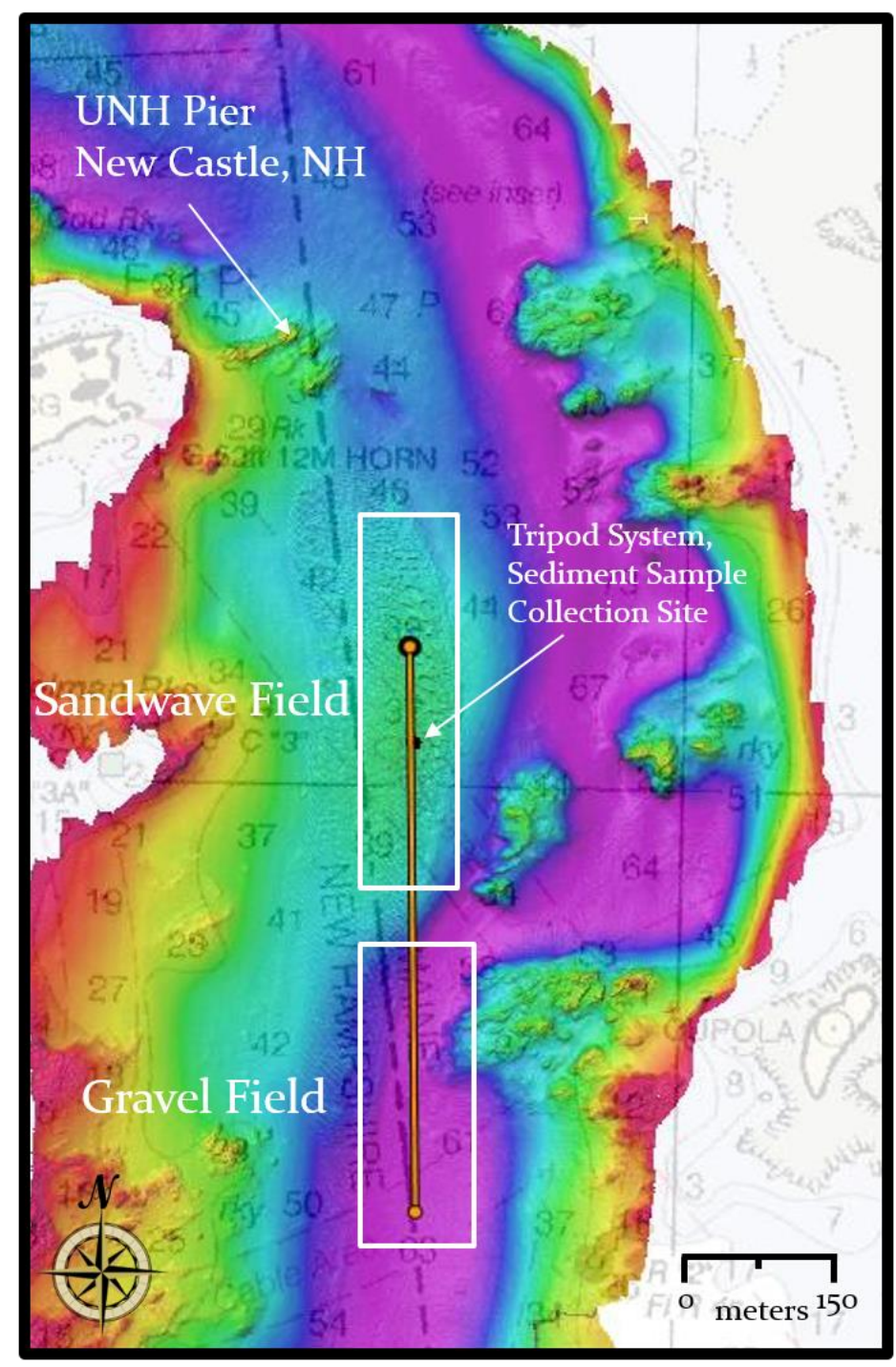

Figure 3-2. Survey transect through sand wave and gravel field.

Both sand wave and gravel field locations had been sampled extensively prior to data collection and noted for their consistency of sediment grain size distribution from multiple different sampling cruises [Ward and McAvoy, 2014]. The sand wave field consisted of very poorly sorted to moderately well sorted, slightly granular medium sands with high shell hash content. This classification was made from sediment samples with grain size distributions that ranged from grain diameters of $0.0625 \mathrm{~mm}$ to $4 \mathrm{~mm}$ with an overall mean grain size of $\sim 0.5 \mathrm{~mm}$. 
The larger grain sizes $(0.707 \mathrm{~mm}$ to $4 \mathrm{~mm})$ were generally comprised of shell hash and were responsible for a significant portion ( 20\%) of the samples. The gravel field consisted of very poorly sorted sandy pebble gravels or pebble gravels. Grain size diameters in the gravel field ranged from $0.0625 \mathrm{~mm}$ to $32 \mathrm{~mm}$ with an overall mean grain size of $\sim 12 \mathrm{~mm}$. Video data screen grabs taken from sediment sampling cruises found in Figure 3-3 provide visual interpretation of the seafloor composition at each major site.
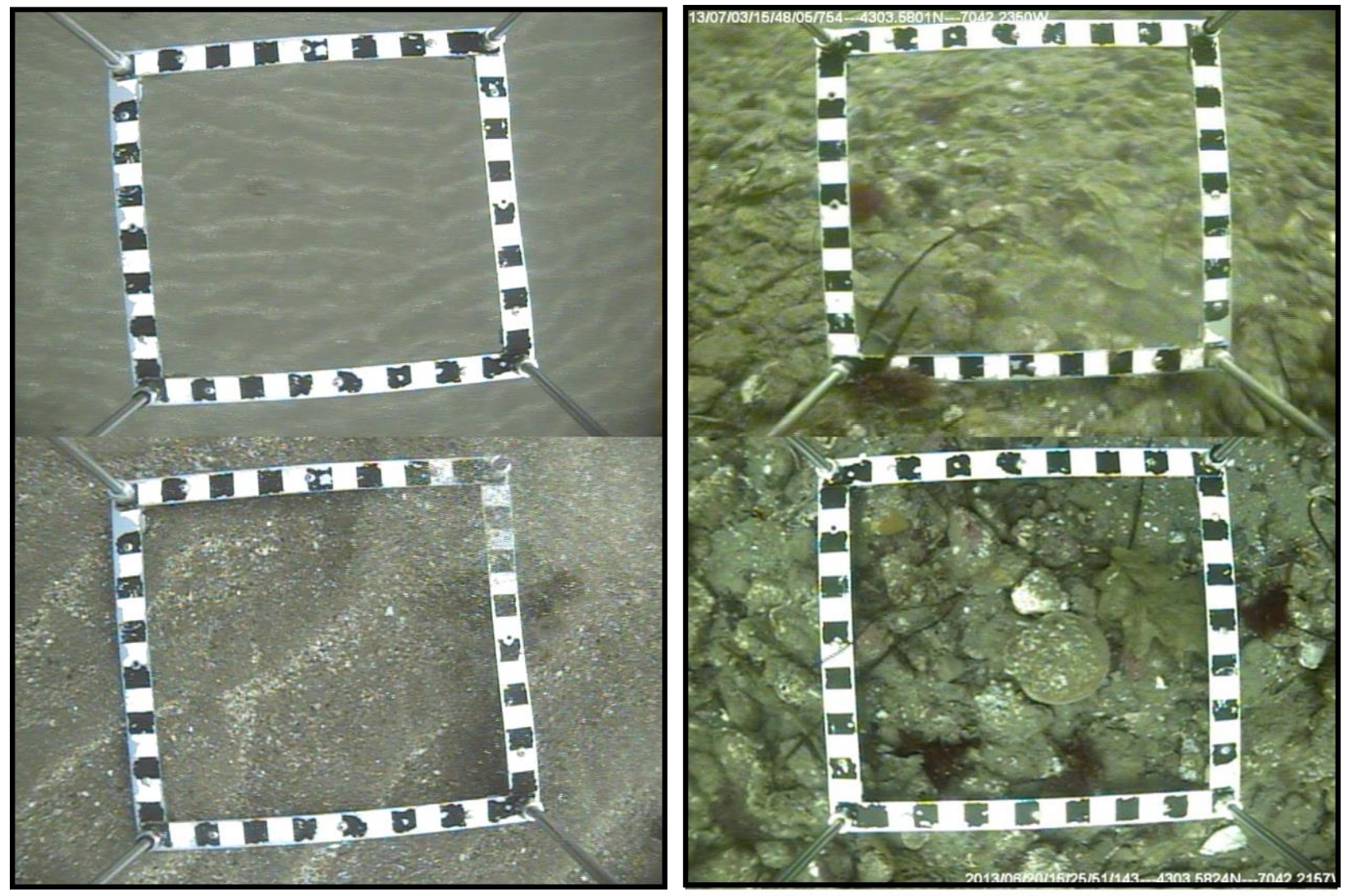

Figure 3-3. Screen grab video data of sand wave and gravel field. Images from the sand wave field are found in the left panel and images from the gravel field are found in the right panel. Note that in the top left image, the camera is above the seafloor. The camera scale present in each image is roughly $0.5 \mathrm{~m}^{2}$.

\section{Current Observations}

The presence of strong tidal currents in Portsmouth Harbor was also of particular interest in the region of the sand wave field due to the smaller grain size composition and high 
probability of sediment transport. Tidally influenced sediment transport would result in a composition change of the seafloor that would possibly lead to a change in the overall statistics of the seafloor roughness spectrum. Changes in the roughness spectrum would affect the theoretical prediction of seafloor backscatter related to small-roughness perturbation theory discussed in Chapter 2.1. Therefore, an ADCP current profiler (1200 kHz RDI Workhorse Sentinel) was deployed via the tripod system within the sand wave field to characterize the magnitude and direction of the current during the survey period utilized for comparison to sediment transport theory provided by Felzenburg [2009].

The tripod system was deployed on the eastern periphery of the sand wave field at approximately $43.067^{\circ} \mathrm{N}, 70.704^{\circ} \mathrm{W}$, (Figure 3-2) utilizing an A-frame and winch system. Current observations from the ADCP were truncated to 6:34 PM UTC through 10:14 PM UTC to synchronize results to acoustic backscatter observations. The truncated time period in which data collection occurred is outlined in Figure 3-4.

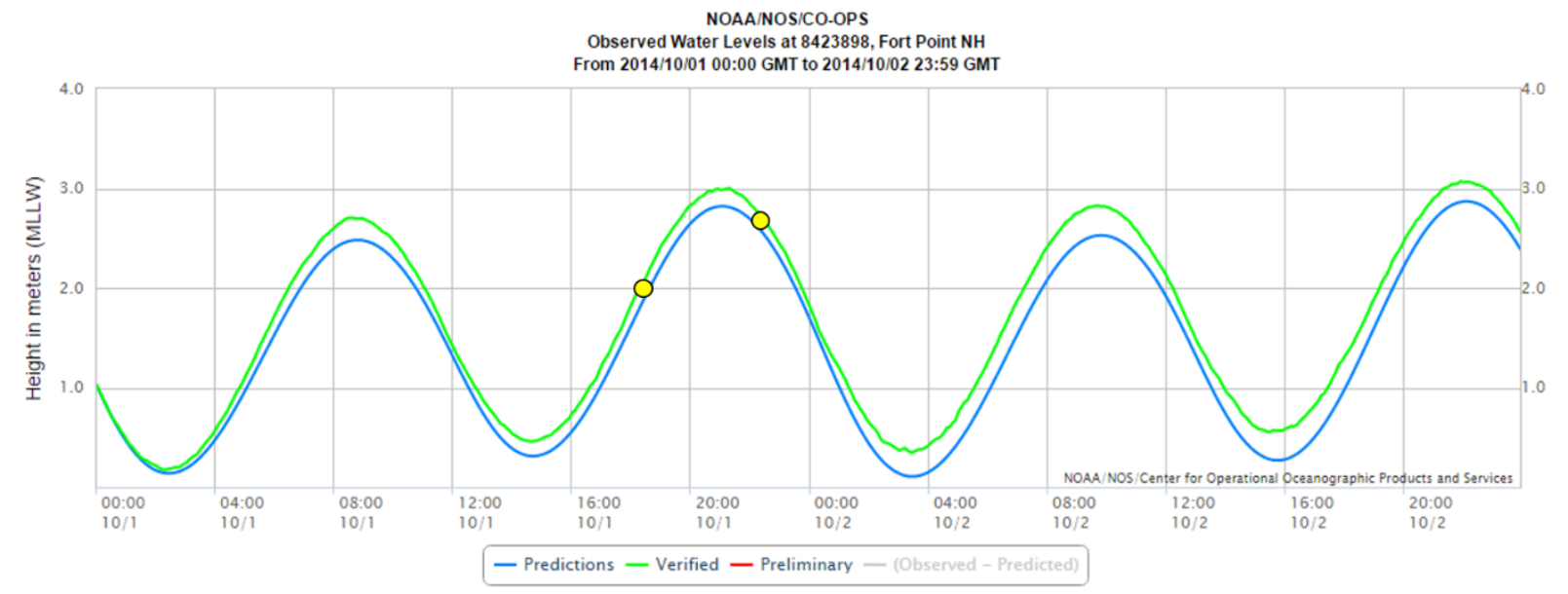

Figure 3-4. Time series of tide elevation during the 24 hour period surrounding the field experiment. Yellow dots indicate the start and stop periods of data collection [NOAA, 2015]. 
ADCP current speed and direction were recorded from $1.55 \mathrm{~m}$ above the bottom to the free surface of the water during a flood tidal cycle and are presented in Figure 3-5. Average depth of the water was computed to be $13.3 \mathrm{~m}$ by the ADCP over the survey period.

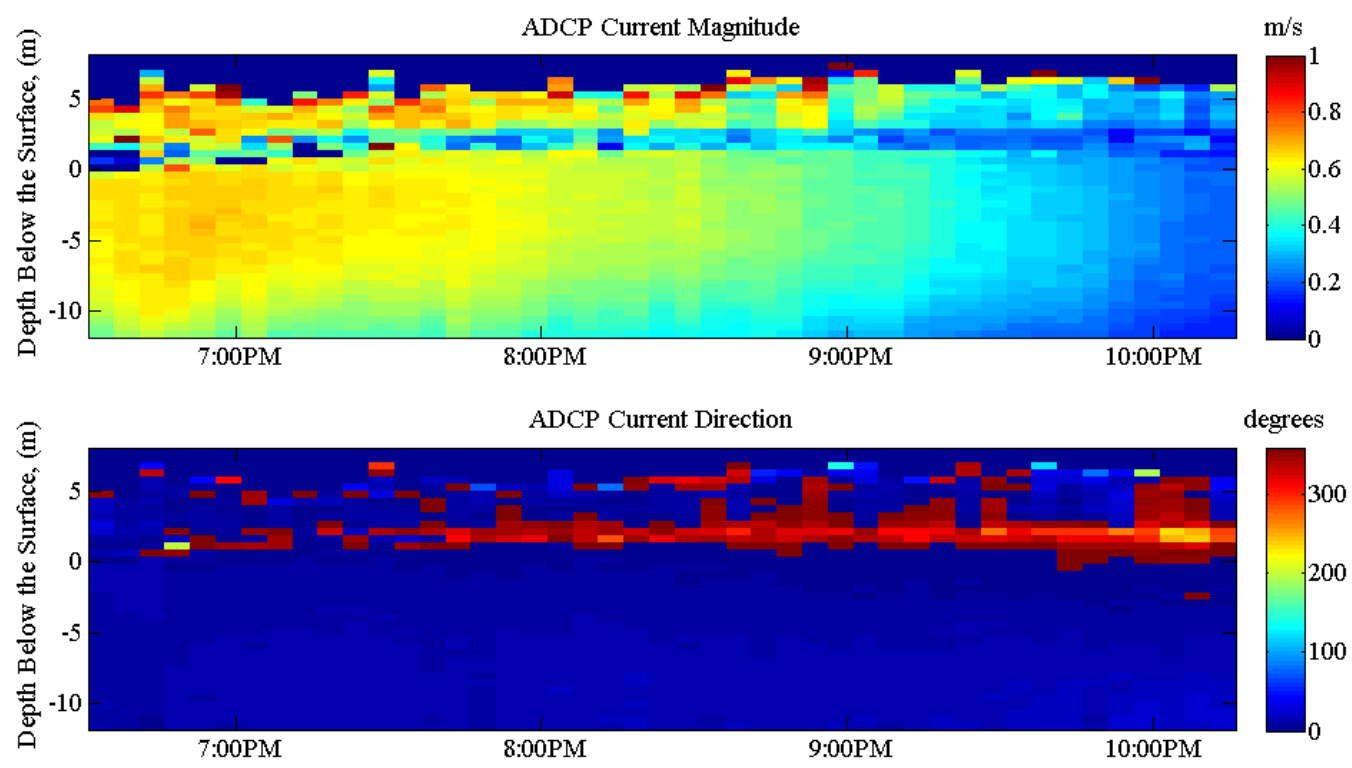

Figure 3-5. Time-series of ADCP current speed in $\mathrm{m} / \mathrm{s}$ and direction is degrees. The nominal sea surface is at $0 \mathrm{~m}$ and the tripod was deployed at $\sim 12 \mathrm{~m}$. Depths are shown as negative numbers on the $y$-axis. Direction of the tide was north flowing represented by $0^{\circ} / 360^{\circ}$.

The time series of the ADCP current profile shows an increase in current speed with height above the bottom. Maximum current speeds of $\sim 55 \mathrm{~cm} / \mathrm{s}$ closest to the seabed, were reached at the beginning of the survey and decayed to approximately zero by the end of data collection. Comparison of the current profile data to subset sections of flood tide data collected by Felzenburg [2009] (Figure 3-6), also on the eastern periphery of the sand wave field, suggested that adequate current velocities to initiate incipient motion of the sediment were reached during the survey period. 

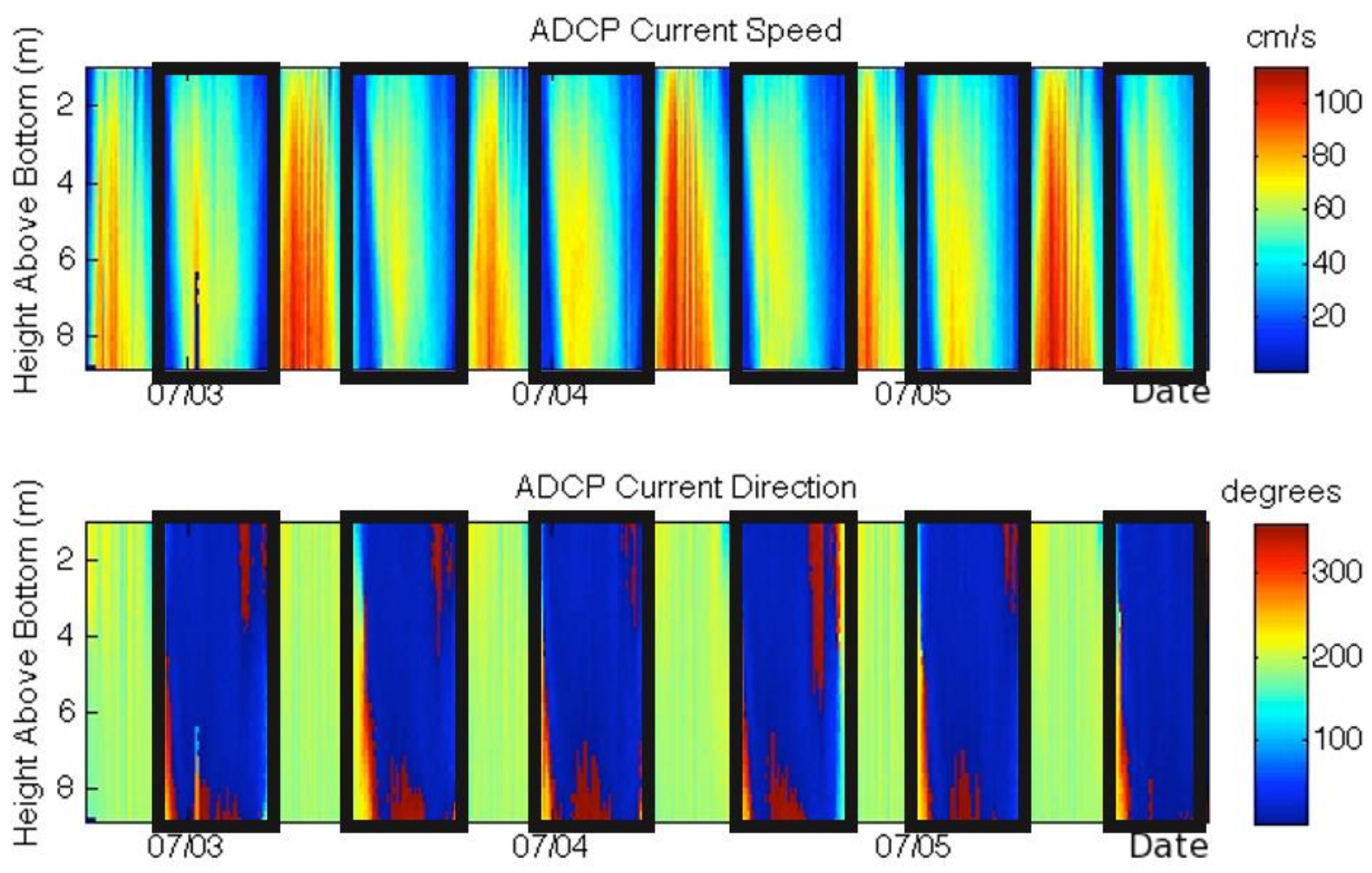

Figure 3-6. Time-series of ADCP current speed (in $\mathrm{cm} / \mathrm{s}$ ) and direction (in degrees) for all bins, corresponding to 1.03 to $8.83 \mathrm{~m}$ above the bottom. Flood currents are north-flowing (dark blue/red in the bottom image) and ebb currents are south flowing (green) [Felzenburg, 2009]. Flood current events are outlined by black boxes.

Maximum current speeds closest to the seabed, during flood tide events were $\sim 54 \mathrm{~cm} / \mathrm{s}$ according to Figure 3-6. Felzenberg [2009] predicted levels of shear stress from near bottom current velocities that exceeded the critical threshold for incipient motion based on the size distribution of the sediment and current velocity profiles within the sand wave field. The combination of consistency in current profile data and grain size distribution estimates from data sets collected for this study to data sets provided by Felzenburg [2009] and Ward and McAvoy [2014] added confidence to the assumption that sediment in the sand wave field was in fact in motion during high current velocity periods of the acoustic survey. 


\subsection{Data Collection and Results}

\section{Stereo Imagery Data: Surficial Roughness Scattering}

An underwater stereo photography system was also deployed with the tripod system to collect simultaneous imagery data within the sand wave field during the SBES survey to provide quantitative description of the surficial roughness of the seafloor needed as input to the roughness scattering model. Stereo imagery data was also collected in the gravel field following the acoustic survey. Divers assisted in the deployment of the tripod system to ensure proper positioning of the camera's field of view (FOV) perpendicular to the current direction. Positioning of the stereo camera system was important to avoid local scouring effects from the instrumentation itself if the current were to flow parallel to the tripod structure. Two HackHD cameras, separated by a baseline of $40 \mathrm{~cm}$, at a fixed focal length of $1 \mathrm{~m}$ from the bed, were used to construct the stereo camera system. The stereo camera system was mounted to a weighted tripod by a cantilevered arm that extended $1 \mathrm{~m}$ beyond the structure. The cantilevered arm was supported by aluminum piping structures angled at $30^{\circ}$ that were attached to the tripod itself (Figure 3-7). 


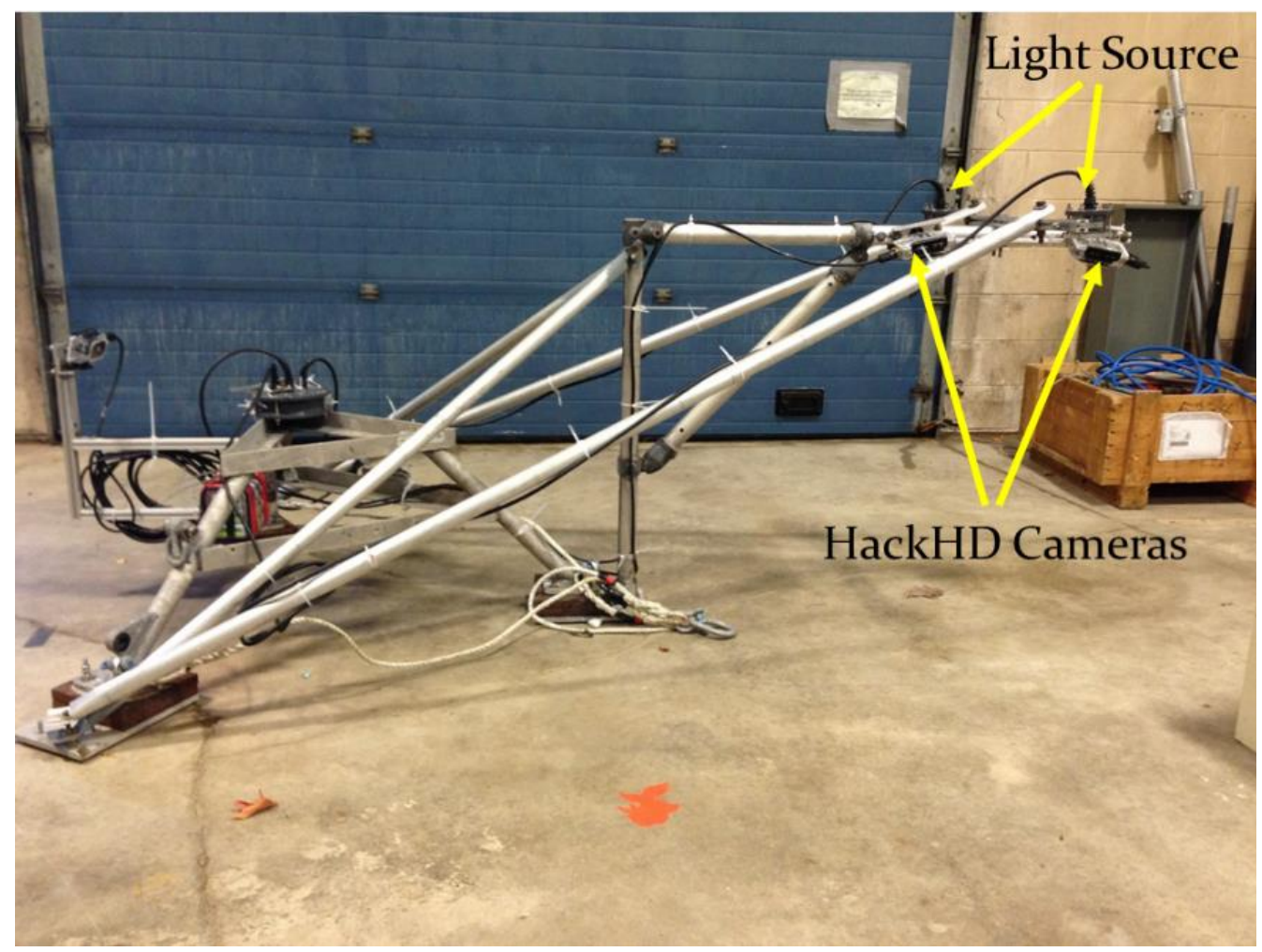

Figure 3-7. Tripod system with mounted stereo camera system.

GoPro Eye of Mine underwater stereo cases were used to house each camera and were mounted rigidly to the tripod system as shown in Figure 3-8. Small LED light panels were used as a light source to illuminate the seafloor and enhance image quality.

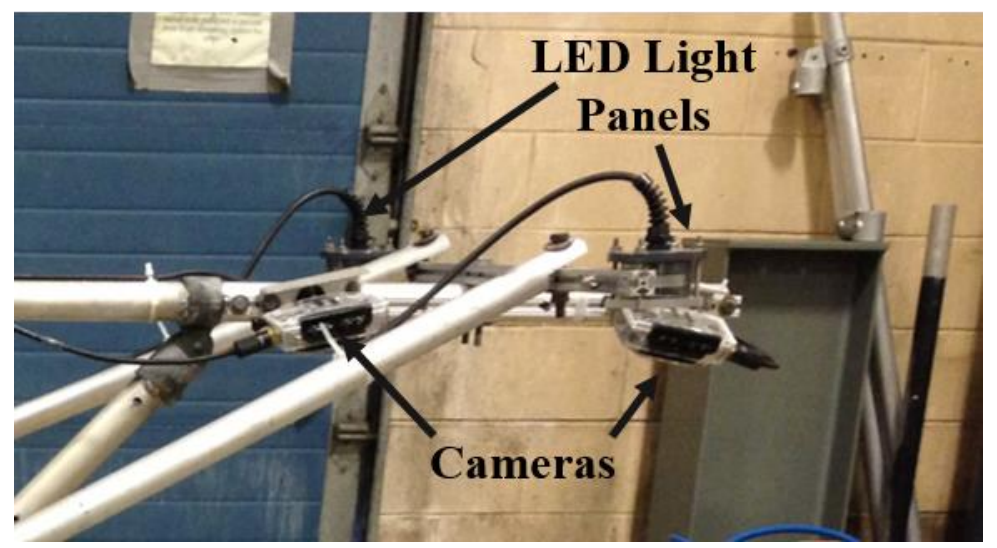

Figure 3-8. Close-up view of cameras and LED light panels rigidly mounted to cantilevered arm attached to the tripod. 
The cameras were tilted at an angle of $10^{\circ}$ with respect to the horizontal axis to ensure overlap of the seafloor within the FOV of each camera during acquisition of the images. The overlapped section of the FOV was used to recreate 3D representation of stereo image pairs. Two 4.00 millimeter $80^{\circ}$ (diagonal FOV) camera lenses were used, allowing for an acquired combined FOV of approximately $\sim 1 \mathrm{~m}^{2}$ (Figure 3-9). The cameras recorded images at a rate of six pictures per minute over the course of a 4-hour survey period, totaling 1,325 stereo pairs.

Side View

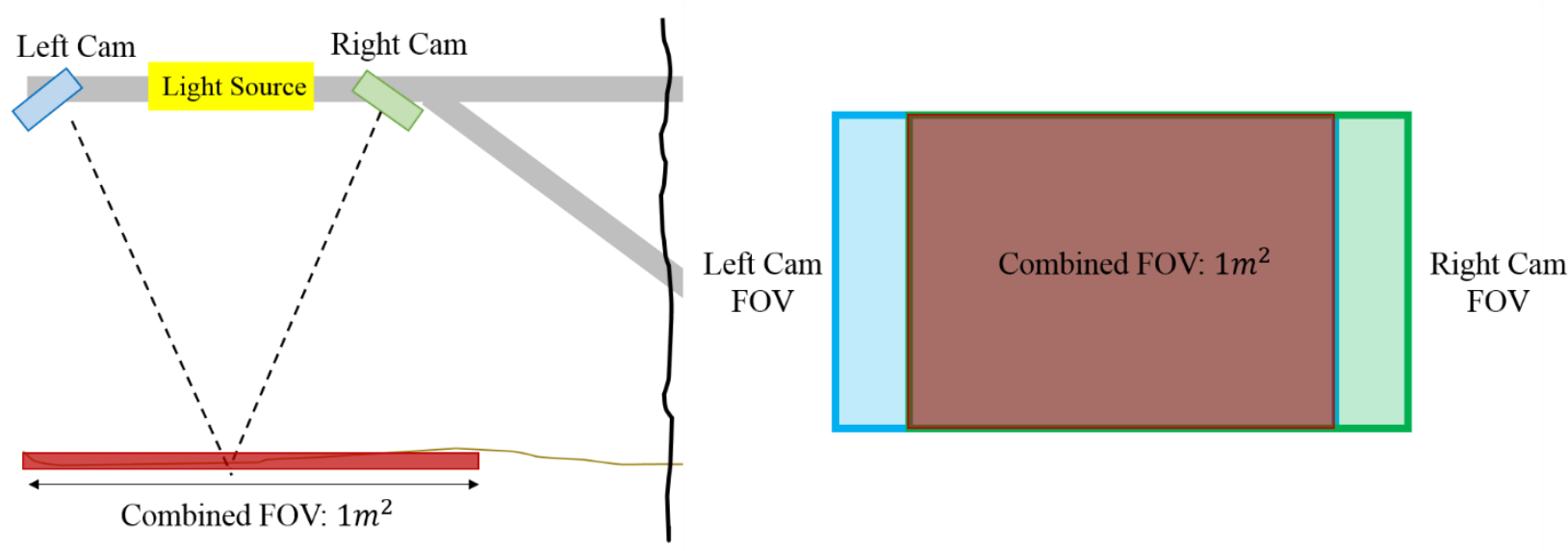

Figure 3-9. Illustration showing overlap FOV of stereo camera system due to the $10^{\circ}$ tilt of each camera towards the center of the baseline.

The stereo camera system was also calibrated before its use in data collection to account for lens distortion. The calibration process was carried out by divers in situ during deployment of the system. This was done to guarantee minimal changes in each cameras' orientation from the start of the calibration process to the end of data collection.

Diver calibration duties consisted of holding a $1 \mathrm{~m}^{2}$ black and white checkerboard pattern (Figure 3-10) within the FOV of the cameras to collect unique images of the calibration target. The checkerboard pattern was created using PowerPoint ensuring a uniform pattern of $50 \mathrm{~mm}$ alternating black and white squares. It was printed and laminated before being attached to a 1/4" 
thick sheet of aluminum. After the process was complete, the divers moved the tripod system away from the calibration site to the top of a dune where the seafloor was undisturbed.

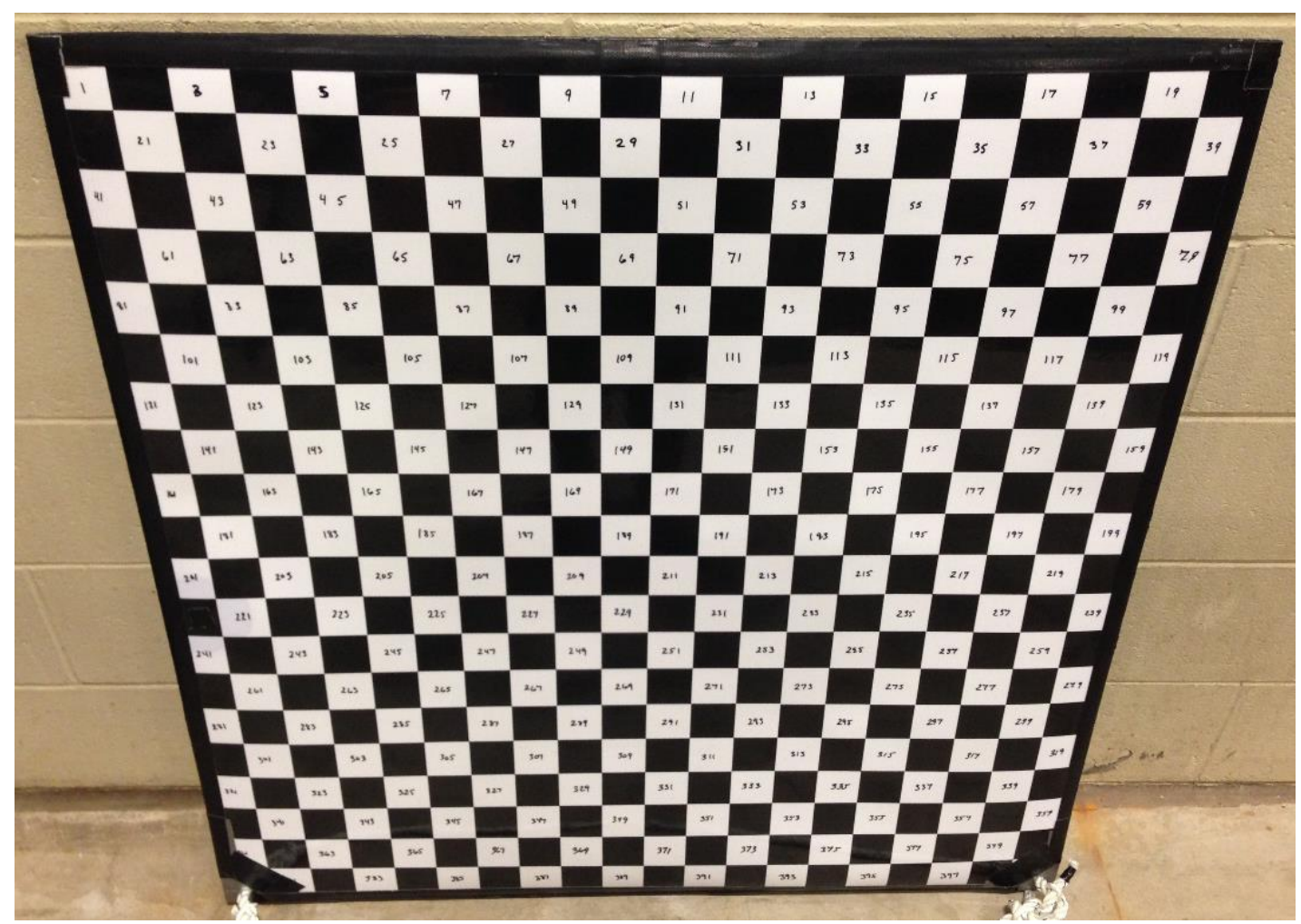

Figure 3-10. Stereo camera calibration target.

The stereo camera system was calibrated to correct for lens distortion evident Figure 3-11. This distortion was corrected in order to construct three dimensional point clouds, or digital elevation models (DEM) from a given stereo image pair necessary in determination of the roughness spectrum. Using a tool box provided by the California Institute of Technology [Bouguet, 2013] calibration image pairs were used to develop correction parameters for both left and right images of the data set. Correction parameters were then used in conjunction with the program LensCorrect, developed at the Center for Coastal and Ocean Mapping/Joint Hydrographic Center (CCOM/JHC), to remove the curve effects caused by the lens [private 
communication]. Straight lines were drawn across corrected images to verify the calibration process and the removal of lens distortion before being applied to the data set. An example of this process can be seen in Figure 3-11.

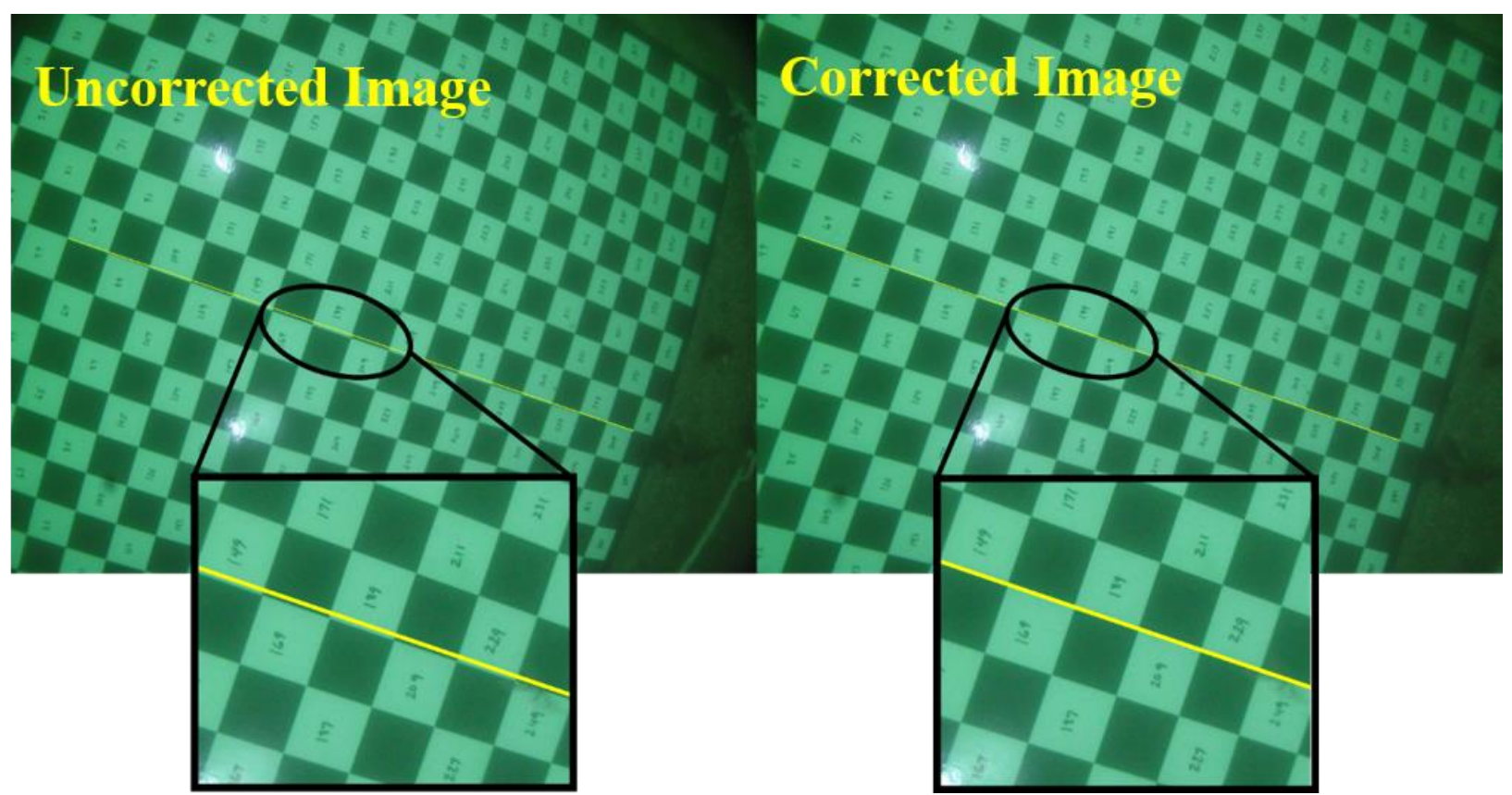

Figure 3-11. Example of uncorrected and corrected calibration images taken from stereo imagery data set. The straight yellow line that is collinear with the connection of black and white squares verifies the removal of curved lens distortion.

DEM's are generated by matching corresponding points common to each image of a stereo image pair [Lyons et al., 2002]. This process becomes possible following the transformation of a given set of stereo images to a common plane, known as rectification. The rectification process determines the transformation of each image's plane, such that conjugate epipolar lines become collinear and parallel to one of the image axes [Lyons et al., 2002]. Rectification reduces the computational work load on DEM generation from a two dimensional search process to a one dimensional search process [Lyons et al., 2002]. Parameters relevant to this process were also determined by the toolbox provided by Caltech. 
Software developed by the University of Stuttgart, Germany was utilized to perform both rectification and automatic stereo-correlation for each corrected image pair [Rothermel et al.]. Automatic stereo-correlation is the process of determining the position of corresponding points on two images to generate a DEM [Lyons et al., 2002]. A technique known as area based matching was used to complete automatic stereo-correlation. Area based matching searches a sub-window within a larger window within a given stereo pair, until correlation between two points is maximized. The two points that correspond to the maximum correlation are considered the same point in each image [Lyons et al., 2002]. This process is repeated until the entire image has been searched, and a DEM has been created. The DEM can be considered as a bathymetric map of each stereo pair. See appendix for post processing details.

DEM results from the sand wave field were first compared to large scale MBES results from Felzenburg [2009] to verify stereo image processing techniques generated accurate results. The stereo camera system was positioned roughly on top of a sand dune, thus the larger scale bedforms from the MBES data were expected to compare relatively well to the stereo imagery data. Large bedforms evident in MBES data ranged from $10 \mathrm{~cm}$ to $50 \mathrm{~cm}$ in height [Felzenburg, 2009], which were comparable to DEM results of the single sand dune visible within the FOV of the stereo cameras. The sand dune decreased from $22 \mathrm{~cm}$ in elevation to $13 \mathrm{~cm}$ in elevation over the course of the survey. Elevation change of the large bedform at 15-minute intervals during the most dynamic part of the survey period ( 6:35 PM UTC - 8:00 PM UTC; Low Tide - 2:42 PM UTC, High Tide - 8:54 PM UTC ) is shown in Figure 3-12. Changes in elevation of the sand dune imply sediment motion due to tidally influenced currents within the sand wave field as suggested by Felzenburg [2009]. 

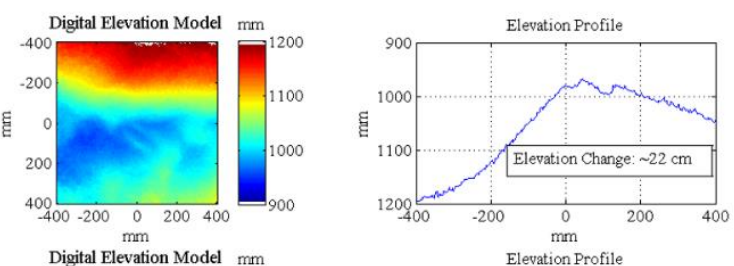

Approximate Survey
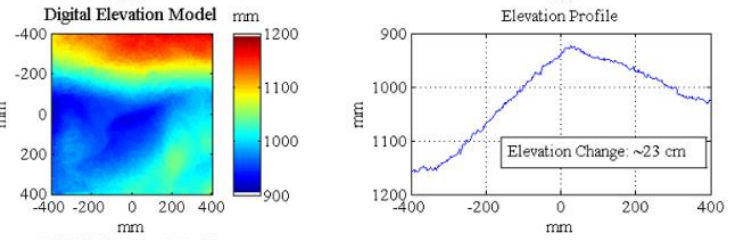

Time

6:35 PM UTC
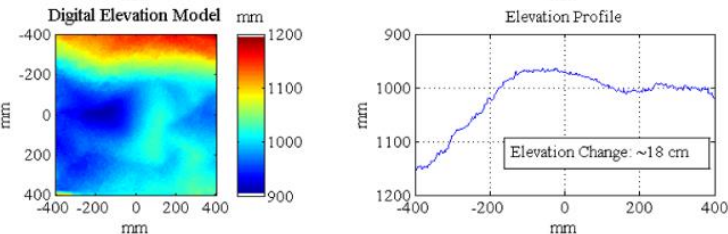

Digital Elevation Model $\mathrm{mm}$
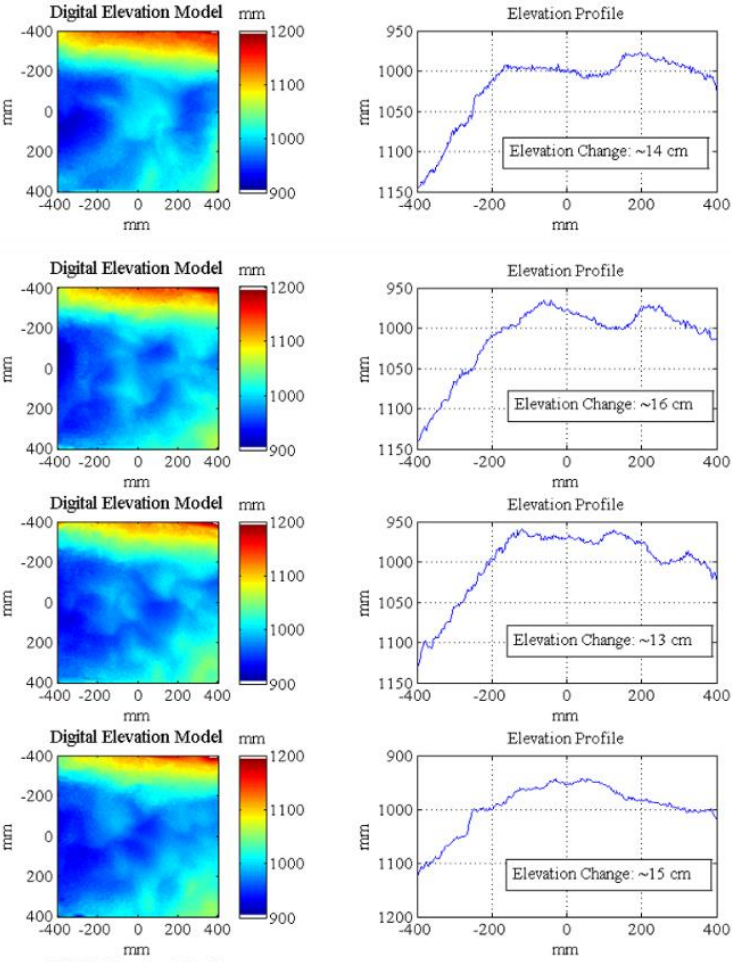

Elevation Profile
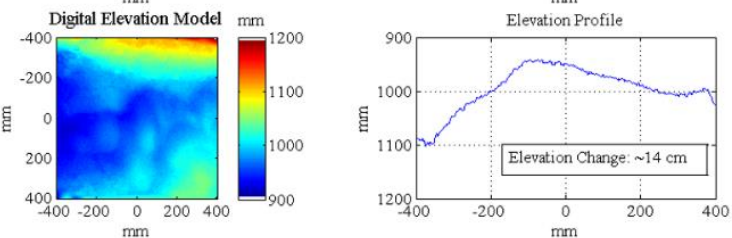

7:15 PM UTC

7:30 PM UTC

7:45 PM UTC

:00 PM UTC

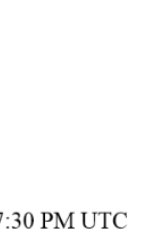

7:45 PMIC

6:45 PM UTC

7:00 PMUTC

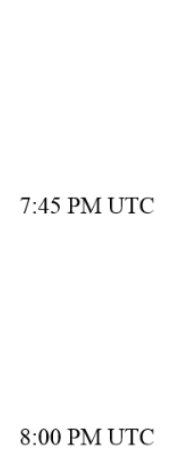

8:00 PM UTC

Figure 3-12. Elevation profiles (right) derived from DEM results (left). Warm colors denote deeper sections of the bedform in each DEM. Elevation change represents the difference from the lowest section of the bedform profile to the highest. 
Total elevation change of the sand dune over the entire survey period is shown in Figure 3-13. The bedform experienced a net decrease in elevation of approximately $7 \mathrm{~cm}$.
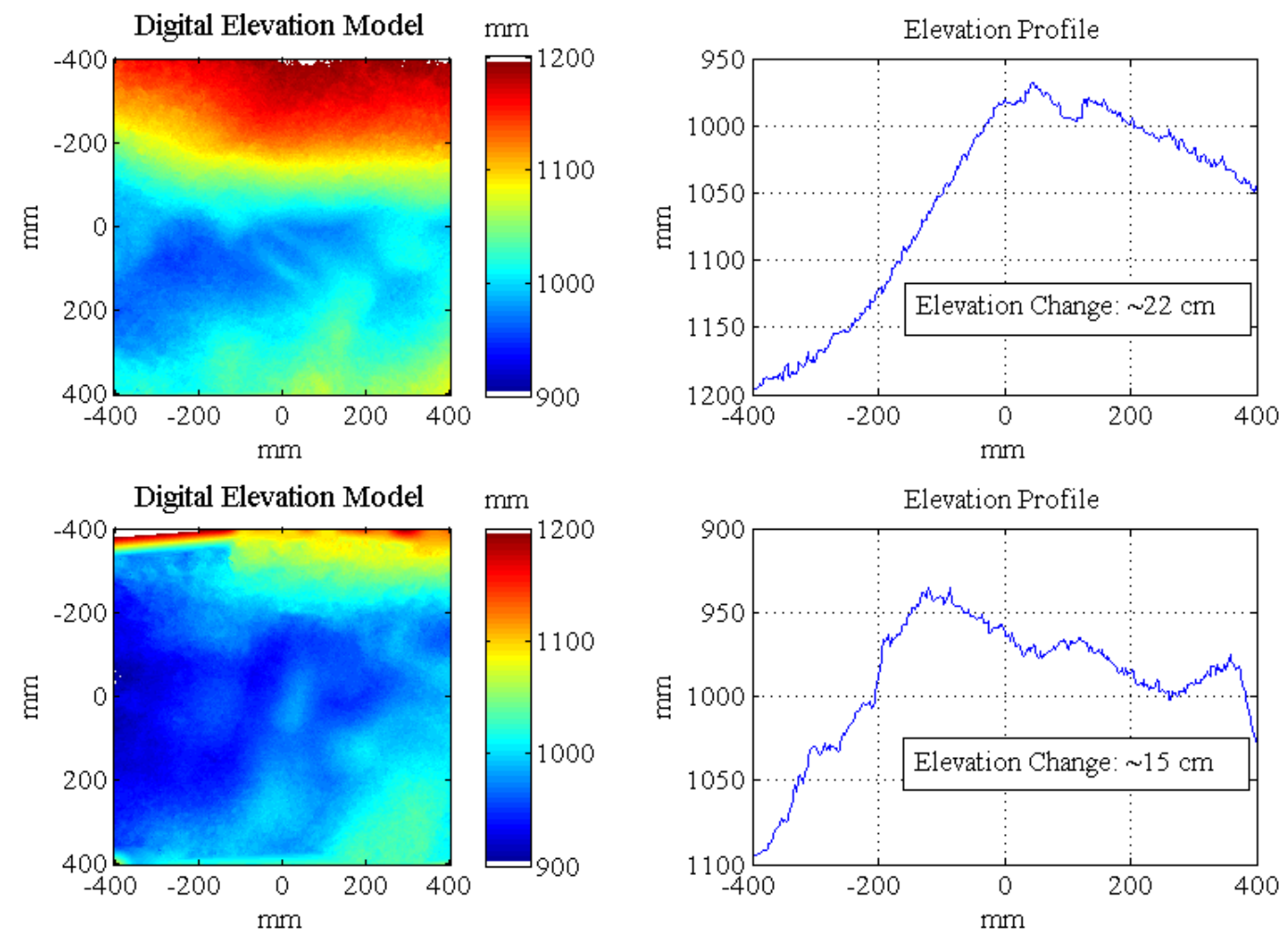

Figure 3-13. Elevation profiles of large bedform observed by the stereo camera system at the start of the survey (top) and the end of the survey (bottom). A total elevation change of $\sim 7 \mathrm{~cm}$ was recorded.

The roughness spectrum of each DEM was computed applying the techniques outlined in Chapter 2.1. However, each spectrum was calculated by averaging horizontal slices taken from 25 individual sub spectra of each DEM (Figure 3-14) in place of a single horizontal slice through the complete spectrum. This was done to prevent the dominant effects of low frequency bedforms present in the complete spectrum of each DEM due to larger bedforms such as the sand dunes and sand ripples located in the FOV of the stereo camera system. The maximum wavelength of the acoustic signal was only $15 \mathrm{~mm}(0.015 \mathrm{~m})$, whereas the sand dunes ranged from $3 \mathrm{~m}-5 \mathrm{~m}$ in wavelength and ripples were approximately $0.20 \mathrm{~m}$ in wavelength 
[Felzenburg, 2009]. Therefore, scattering effects from larger bedforms were negligible in terms of the acoustic data and filtered out of the stereo imagery data.

(A)

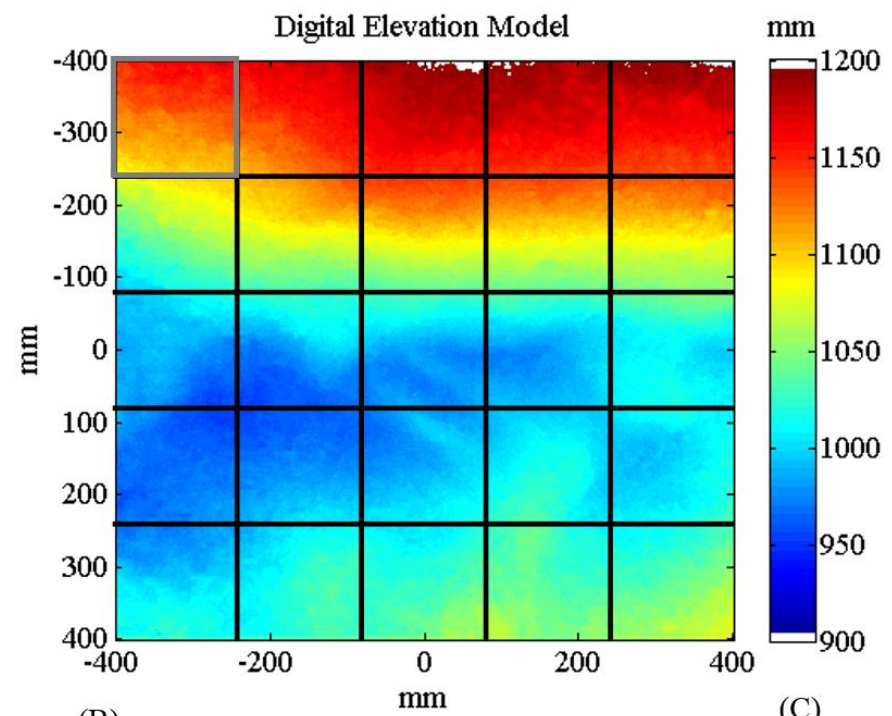

(B)

(C)
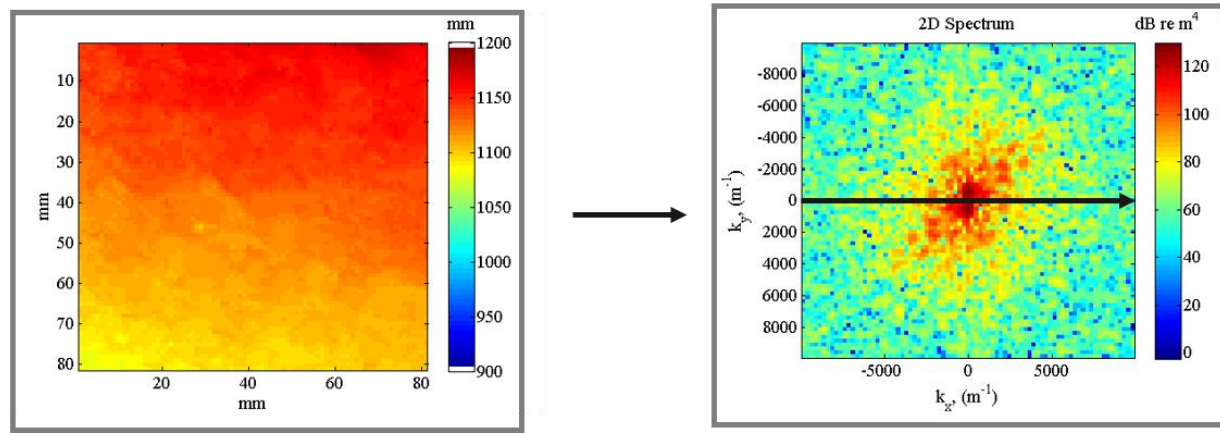

Figure 3-14. Illustration of example calculation of roughness spectrum for stereo imagery data collected in the sand wave field. (A) Division of DEM into 25 evenly spaced sub sections. (B) Corresponding sub section of DEM highlighted in grey in the upper left hand corner of (A). (C) 2D spectrum of (B) where the black line represents the horizontal slice taken from the spectrum equivalent to $1 \mathrm{D}$ integration of the $2 \mathrm{D}$ spectrum.

Although the roughness scattering model assumes the seafloor was isotropic [Jackson and Richardson, 2007], horizontal slices over the 2D spectrum were of interest due to the mounted orientation of the sonar transducers. The beam of each SBES was subject to scattered returns from the horizontal orientation of the spectrum. Directionality of bedforms present on the seafloor is recognized by pronounced directionality of the central peak in a $2 \mathrm{D}$ spectrum. [Briggs 
et al. 2005]. If any directionality was present in the data collected, it was accounted for by applying this methodology.

It is also important to recognize that prior to application of the 2D-FFT, DEM's were fit to a plane to remove any trends in the data and gridded to $2 \mathrm{~mm}$ spacing to ensure uniformly spaced data within the DEM matrix. A Hanning window was also applied to each DEM prior to application of the 2D-FFT to mitigate spectral leakage effects. Spectral leakage can be attributed to the finite size of data segments used in this analysis [Lyons et al., 2002]. A Hanning window was chosen for its low sidelobe levels and fast falloff rate and can be defined as,

$$
\begin{gathered}
w(n)=\cos ^{2}\left[\frac{n}{N}\right]=0.5\left[1.0+\cos \left[\frac{2 n}{N} \pi\right]\right], \\
n=-\frac{N}{2}, \ldots,-1,0,1, \ldots, \frac{N}{2}
\end{gathered}
$$

Equation 18

The Hanning window belongs to the $\operatorname{Cos}^{\alpha}(X)$ family of windows, which are dependent on the parameter $\alpha$ [Harris, 1978]. As $\alpha$ increases, the first sidelobe level decreases from the peak of the main lobe, which improves effects on spectral leakage. However, as $\alpha$ increases, the mainlobe of the window increases, resulting in a loss of frequency resolution. For a Hanning window, where $\alpha=2$, sidelobe levels falloff at a rate of $18 \mathrm{~dB}$ /octave while maintaining a relatively narrow mainlobe. The highest sidelobe level for a Hanning window is $-32 \mathrm{~dB}$ from the peak of the main lobe, whose equivalent noise bandwidth equates to 1.5 frequency bins [Harris, $1978]$.

Results of the roughness spectrum were compared when applying a Blackman-Harris window to the data set, rather than a Hanning window. The comparison was made to ensure bias effects from spectral leakage were at a minimum regardless of window choice. The first side 
lobe level of the Blackman-Harris window was significanlty lower than that of the Hanning window at $-92 \mathrm{~dB}$ falling off at a rate of $6 \mathrm{~dB}$ /octave [Harris, 1978]. However, the main lobe of the Blackman- Harris was wider than that of the Hanning window at 2 frequency bins [Harris, 1978]. Comparison of the roughness spectrum computed using either window proved to vary by only a few hundredths of a decimal place. This result confirms that spectral leakage effects were mitigated equally by either window choice and the data was not biased uniquely by either window.

A total of 1,325 spectra were computed from stereo imagery data over the sand wave field. The behavior of the slope of the roughness spectra, was evaluated independently at the Bragg wavenumber in log-log space, corresponding to both the upper and lower frequency bands of acoustic data collected. The lower band of frequencies corresponded to a resolution range of $\sim 7 \mathrm{~mm}$ to $\sim 10.5 \mathrm{~mm}$. The upper band frequencies corresponded to a resolution range of $\sim 5 \mathrm{~mm}$ to $\sim 6 \mathrm{~mm}$, after the analysis window was cutoff at $225 \mathrm{kHz}(K=1,333 \mathrm{rad} / \mathrm{m})$. This restriction was applied due to the behavior of resultant spectra lines leveling out at frequencies beyond this frequency range. The cutoff region describes the beginning of the noise floor of the system where data at higher frequencies becomes unreliable. The noise floor was most likely caused by high frequency noise near the edges of the 2D spectrum. The cutoff point is highlighted in Figure 3-15. 


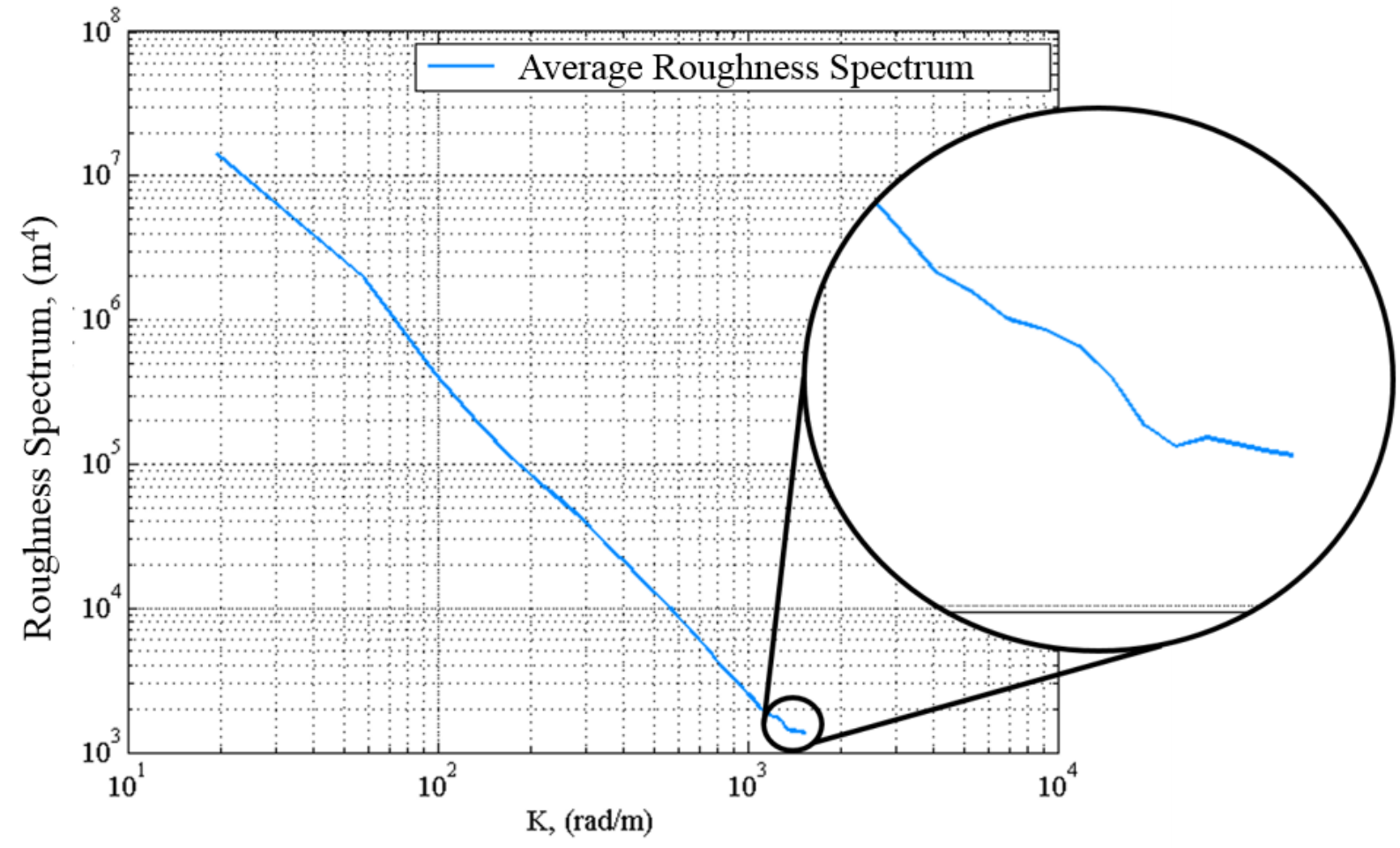

Figure 3-15. Example roughness spectrum that exhibits behavior of leveling out beyond $225 \mathrm{kHz}(\mathrm{K}=1,333 \mathrm{rad} / \mathrm{m})$.

Inspection of individual slope values calculated from each unique spectrum in both upper and lower frequency bands (Figure 3-16) revealed consistency throughout the data set, suggesting spatial stability of the seafloor related to the surficial roughness. Although the seafloor composition appeared to change by visual inspection of the stereo imagery, roughness spectrum results did not. Slope values in each frequency band varied by only a few tenths in magnitude when averaged in time over 3-minute intervals. 

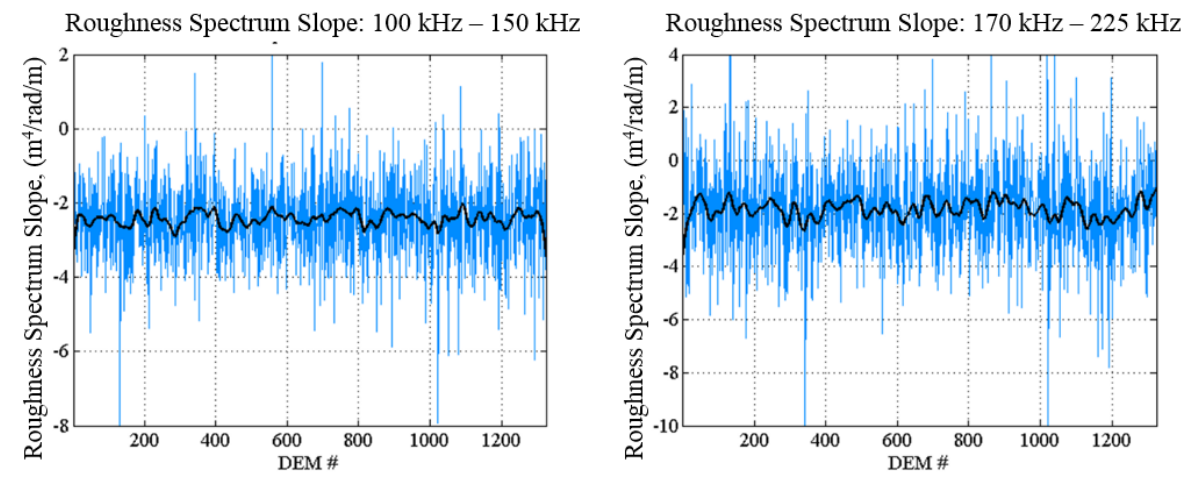

Figure 3-16. Evaluated slopes of roughness spectra for both lower (left) and upper (right) frequency bands. The black line indicates a 3-minute rolling filter of the data in each panel.

However, slope results appear noisy due to the high variability of individual spectra and the short range of frequency bands at which each spectra was evaluated. Variation in slope from consecutive spectra at each frequency band was influenced by subtle changes in spikes inherent to individual spectra evident in Figure 3-17. The spikes were caused by an increase in energy within the spectrum at the corresponding wavenumber. As a result, approximately $7 \%$ of slopes in the upper frequency band and $1 \%$ in the lower frequency band were calculated positive, despite the overall trend of each spectrum possessing a downward sloping behavior.
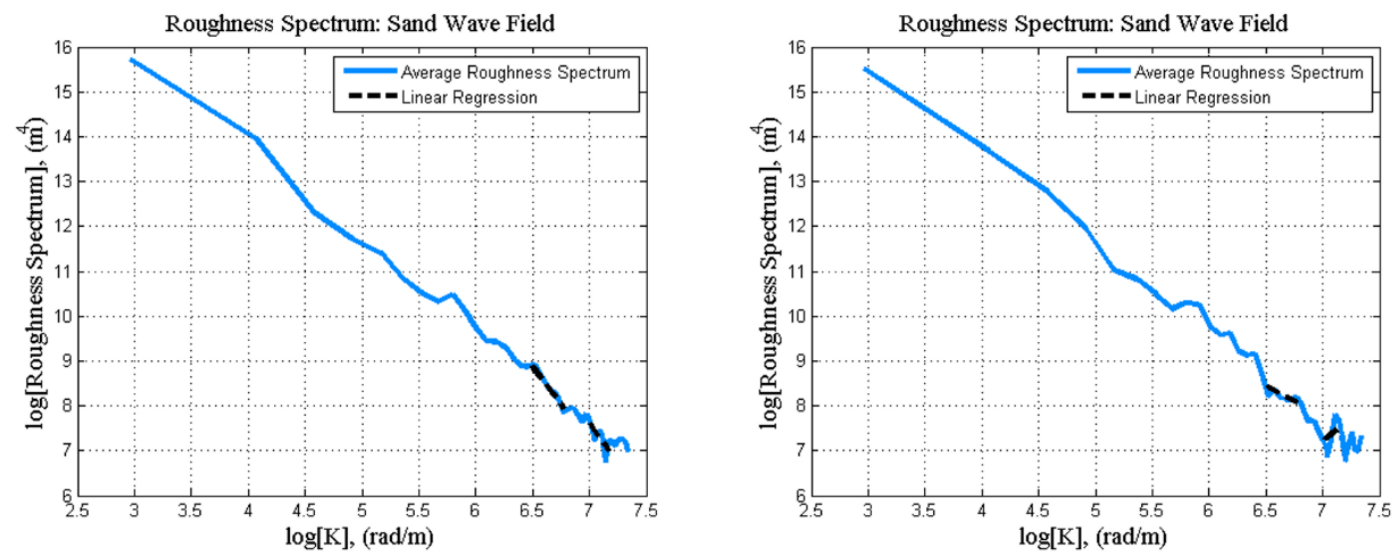

Figure 3-17. Example of two individual spectra calculated from corresponding DEM's. Slopes calculated in both upper and lower frequency bands are noticeably different in each spectrum. Note the positive slope calculation in the upper frequency band shown in the right panel of the figure. 
Due to the consistency of the slope results and the high variability of individual spectra, the resultant spectral exponent required to determine a theoretical prediction of backscatter based on the roughness scattering model, was calculated for each frequency band based on the slope evaluated from all spectra averaged in time. The resultant slope value and corresponding spectral exponent for each frequency band can be found in Table 3-1. Graphical representation of the results is shown in Figure 3-18.

Table 3-1. Average slope and corresponding spectral exponent values for roughness spectrum over the sand wave field.

\begin{tabular}{|c|c|c|c|}
\hline \multicolumn{2}{|c|}{ Lower Frequency Band $(100-150 \mathrm{kHz})$} & \multicolumn{2}{c|}{ Upper Frequency Band $(170-225 \mathrm{kHz})$} \\
\hline Slope & Spectral Exponent, $\gamma_{2}$ & Slope & Spectral Exponent, $\gamma_{2}$ \\
\hline-2.5 & 3.5 & -1.4 & 2.4 \\
\hline
\end{tabular}

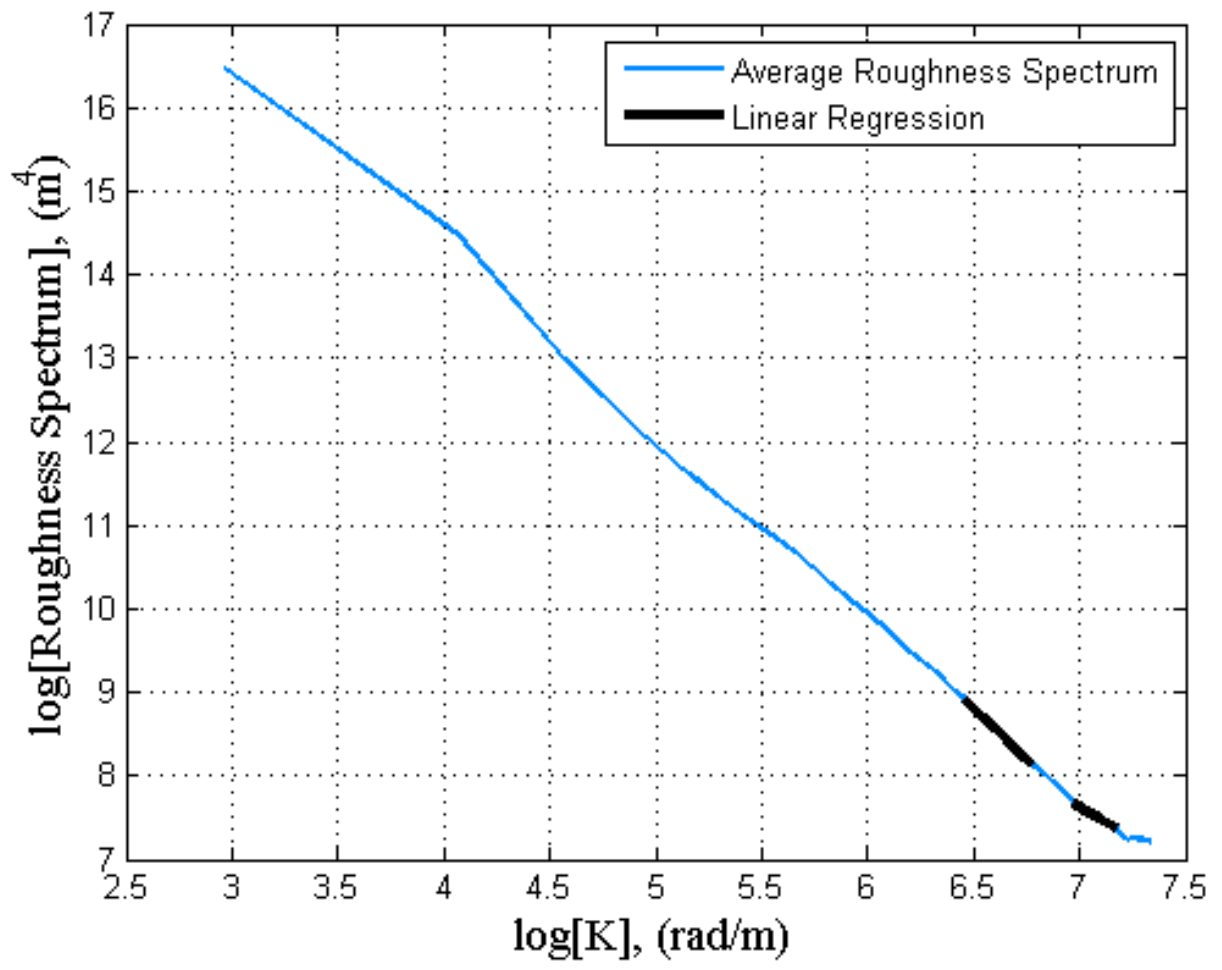

Figure 3-18. Resulting average roughness spectrum over the sand wave field. The solid black lines indicate the linear regression fit of the spectrum at each corresponding frequency band. 
Applying the results from Table 3-1 to Equation 9 suggested that the theoretical seafloor backscatter response computed from data collected over the sand wave field would increase proportional to frequency according to $10 \log _{10} f^{0.5}$ for the lower band of frequencies and $10 \log _{10} f^{1.6}$ for the upper band of frequencies. The theoretical prediction of backscatter for each frequency band is shown in Figure 3-19.

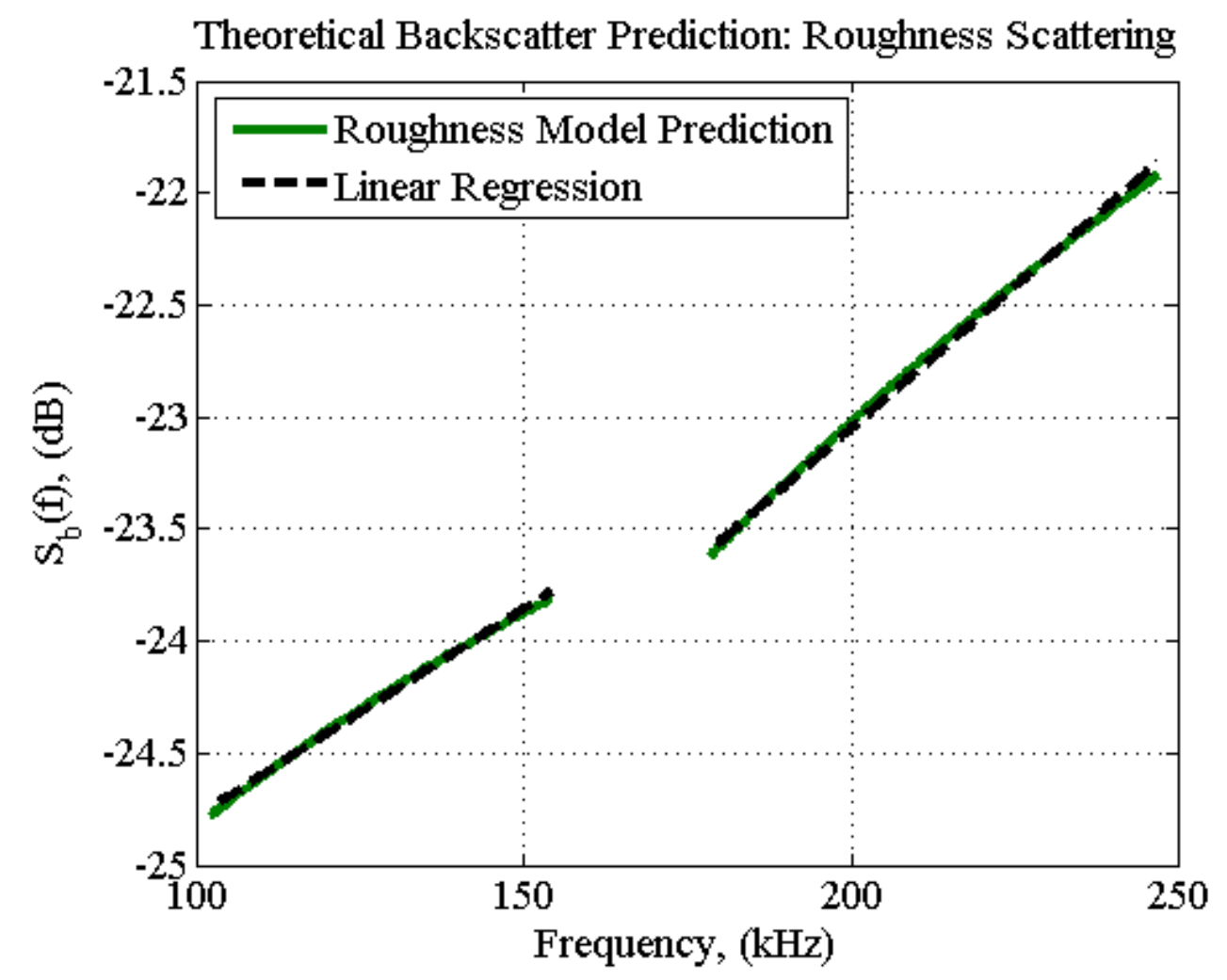

Figure 3-19. Theoretical backscatter prediction for sand wave field produced by roughness scattering model.

Theoretical predictions of backscatter based on the roughness scattering model were also calculated for data collected over the gravel field. An example DEM from the gravel field is shown in Figure 3-20. 


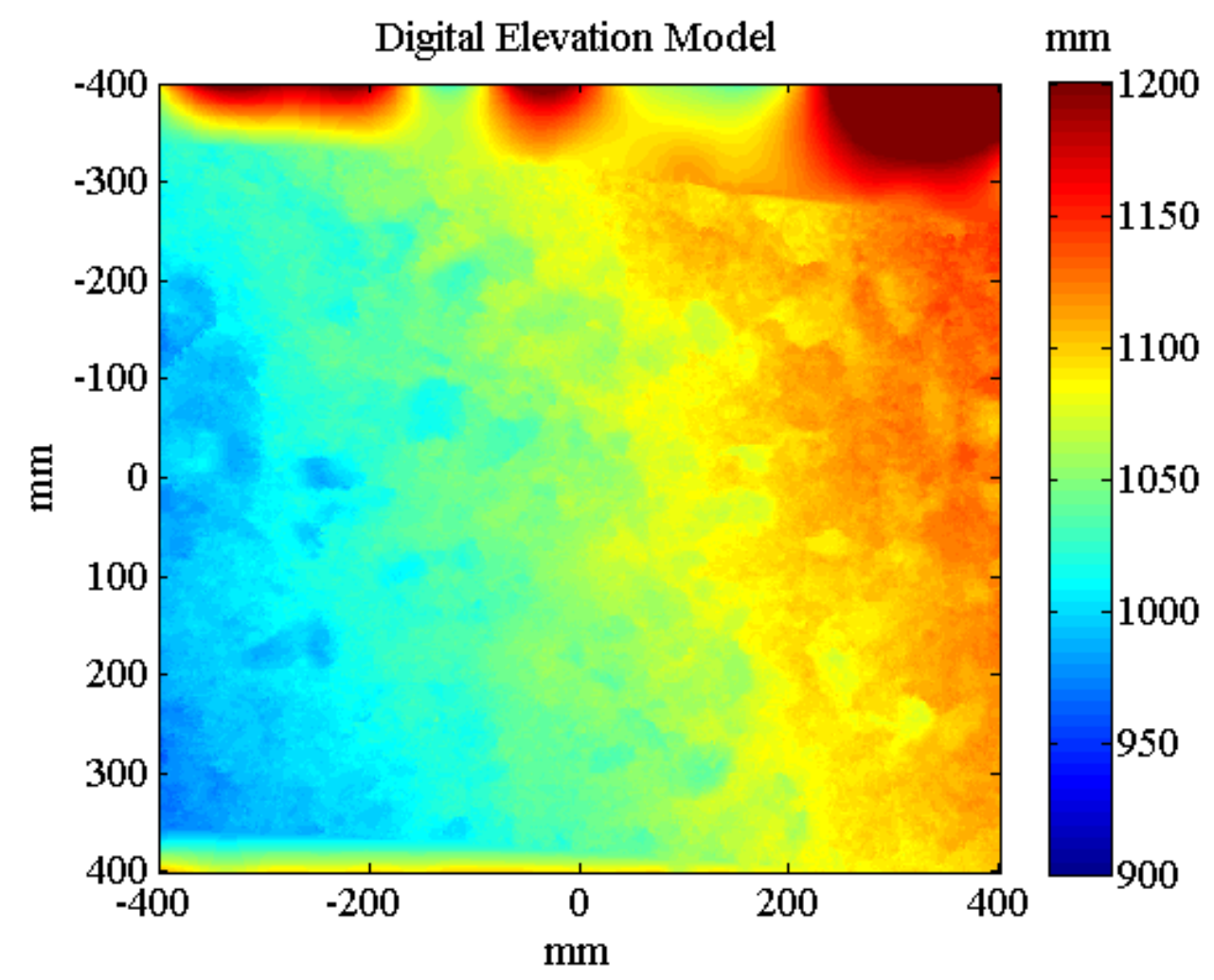

Figure 3-20. DEM example derived from stereo imagery collected in the gravel field.

No current data was collected over the gravel field, therefore sediment transport analysis was not done to predict incipient motion of the sediment. However, the assumption was made that the gravel field remained spatially uniform in time and roughness spectrum statistics would not change. The assumption was made based on the larger sediment composition derived from grain size distribution statistics provided by Ward and McAvoy [2014]. Grain size ranged from $4 \mathrm{~mm}-33 \mathrm{~mm}$ within the gravel field.

Results of individual slopes calculated from individual spectrum derived from images collected in the gravel field possessed similar characteristics to those in the sand wave field. The data appeared noisy due to peaks in individual spectra, yet consistent varying by only and few tenths in magnitude over 3-minute interval averages (Figure 3-21). 

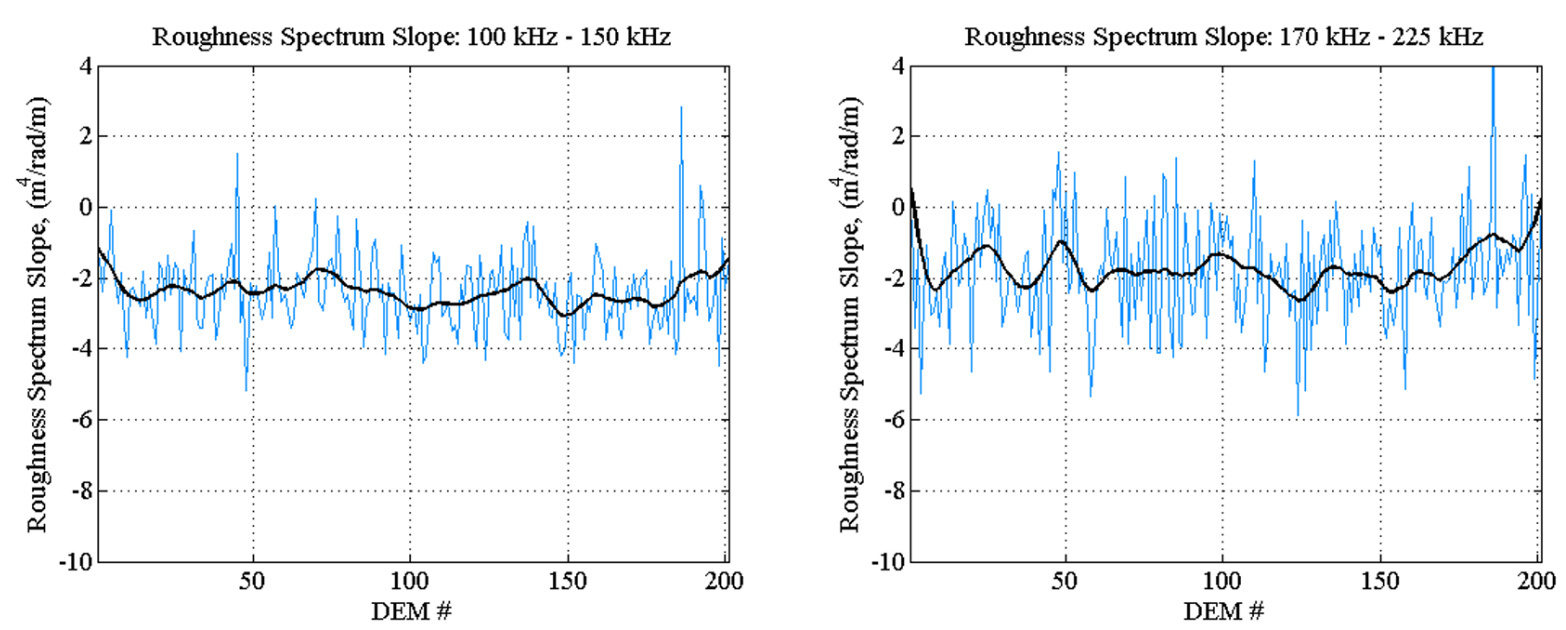

Figure 3-21. Evaluated slopes of roughness spectra for both lower (left) and upper (right) frequency bands. The black line indicates a 3 -minute rolling filter of the data in each panel.

Final results pertaining to the roughness spectra (i.e. slopes and spectral exponents) from data collected over the gravel field were computed after averaging together all individual roughness spectra in time. Slope and spectral exponent values for upper and lower frequency bands are found in Table 3-2. The corresponding roughness spectrum related to the results in Table 3-2 is shown in Figure 3-22.

Table 3-2. Average slope and corresponding spectral exponent values for roughness spectrum over the gravel field.

\begin{tabular}{|c|c|c|c|}
\hline \multicolumn{2}{|c|}{ Lower Frequency Band $(100-150 \mathrm{kHz})$} & \multicolumn{3}{|c|}{ Upper Frequency Band $(170-225 \mathrm{kHz})$} \\
\hline Slope & Spectral Exponent, $\nu_{2}$ & Slope & Spectral Exponent, $\gamma_{2}$ \\
\hline-2.3 & 3.3 & -1.7 & 2.7 \\
\hline
\end{tabular}




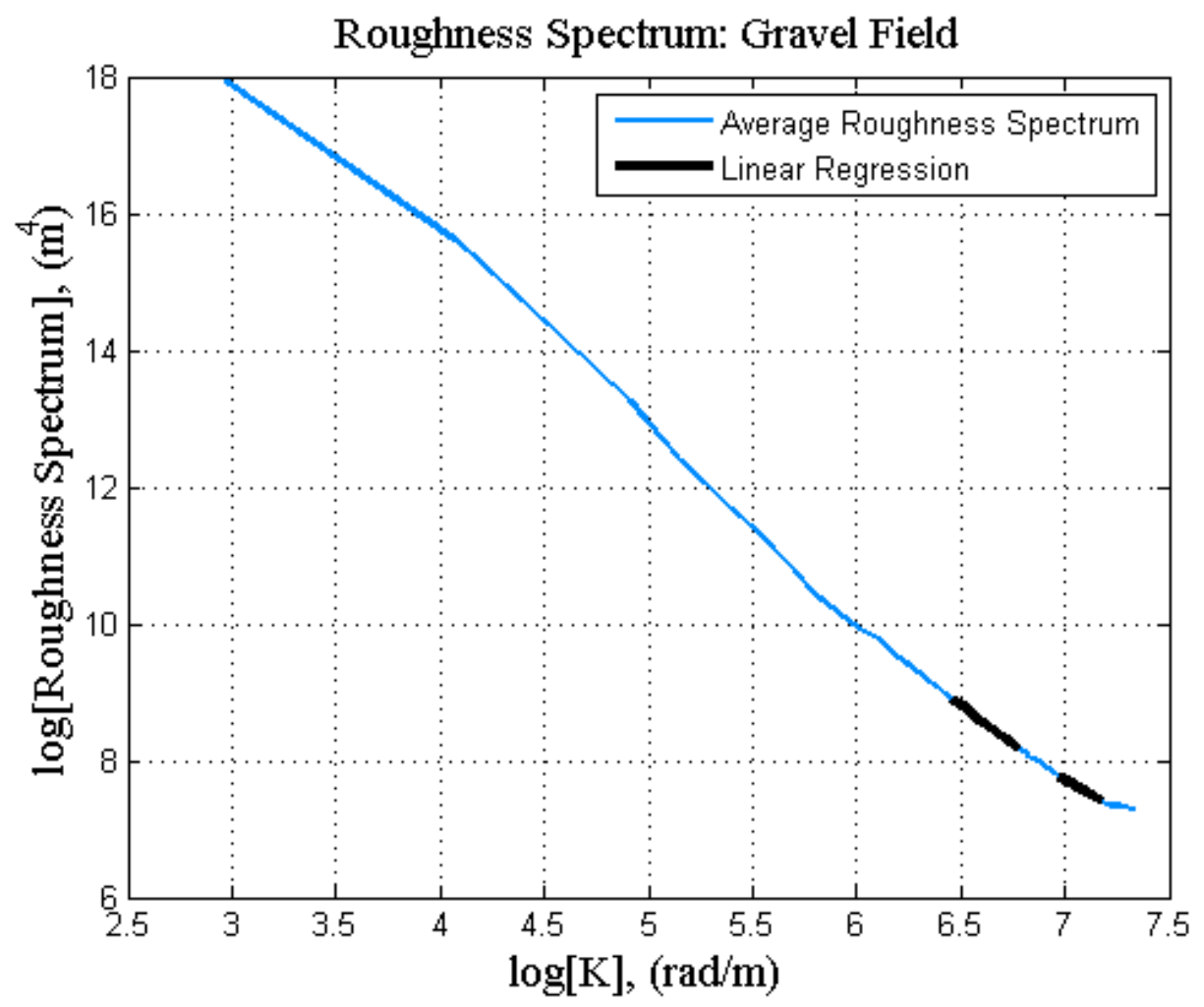

Figure 3-22. Resulting average roughness spectrum over the gravel field. The solid black lines indicate the linear regression fit of the spectrum at each corresponding frequency band.

Applying the gravel field results to Equation 9 suggested that the seafloor backscatter response from the lower band of frequencies would increase proportional to frequency according to $10 \log _{10} f^{0.7}$ and $10 \log _{10} f^{1.3}$ for the upper band of frequencies. The theoretical prediction of backscatter for each frequency band is shown in Figure 3-23. 


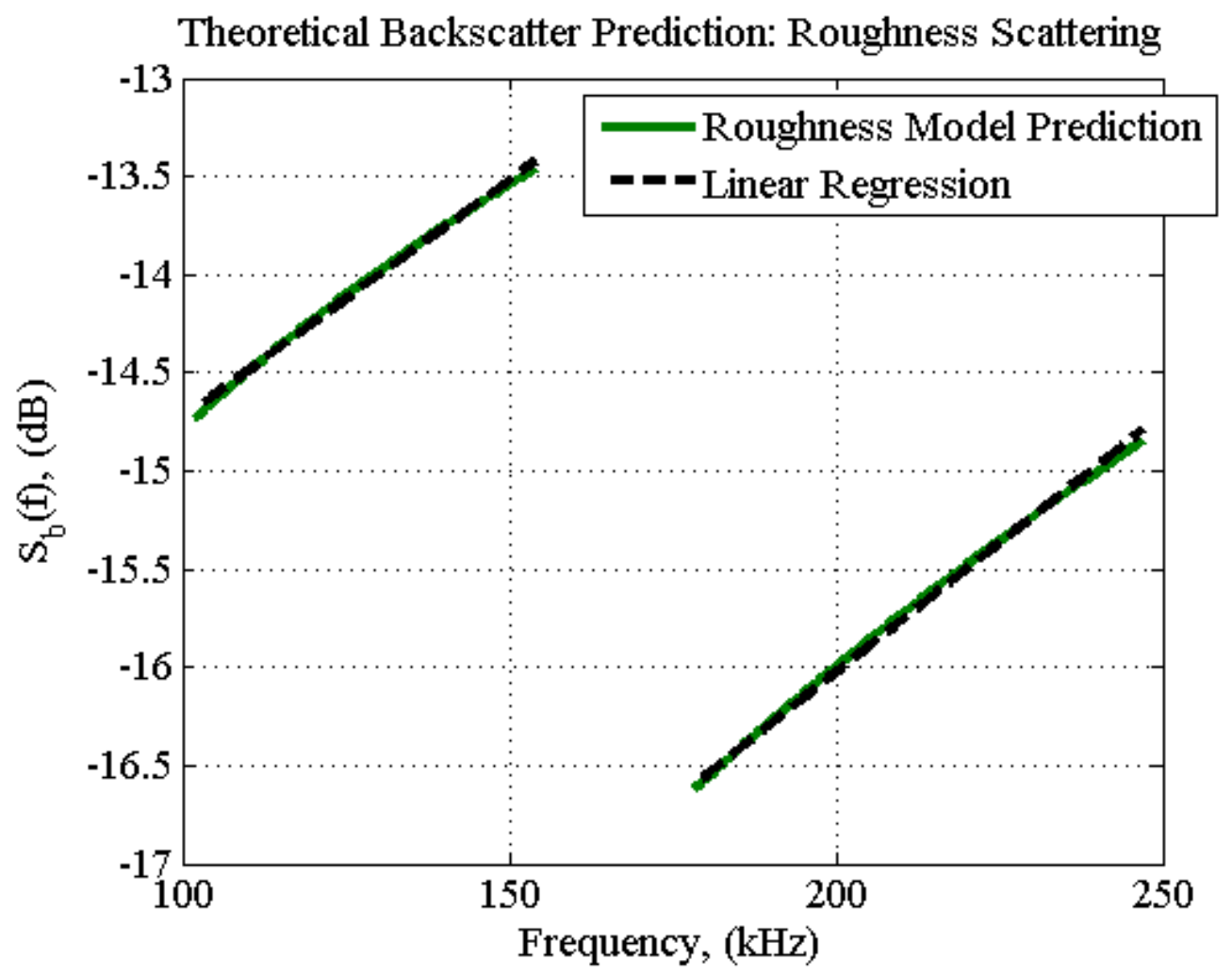

Figure 3-23. Theoretical backscatter prediction for gravel field produced by roughness scattering model.

\section{Sediment Samples: Discrete Inclusion Scattering}

Sediment samples were also collected by divers on site of the tripod system deployed within the sand wave field. Parameters specific to the sediment samples were used in conjunction with the discrete inclusion scattering model described in Chapter 2.2 to generate a theoretical prediction of backscatter to be compared to acoustic observations. The sedimentary composition of the gravel field was not ideal for comparison of acoustic backscatter results to the discrete inclusion scattering model. Divers manually collected grab samples from the top 1.5 " of sediment of a $\sim 10 \mathrm{in}^{2}$ area using their hands. A total of two samples were collected behind the tripod system (Figure 3-24) to ensure an untouched sample area where the stereo imagery was collected. 


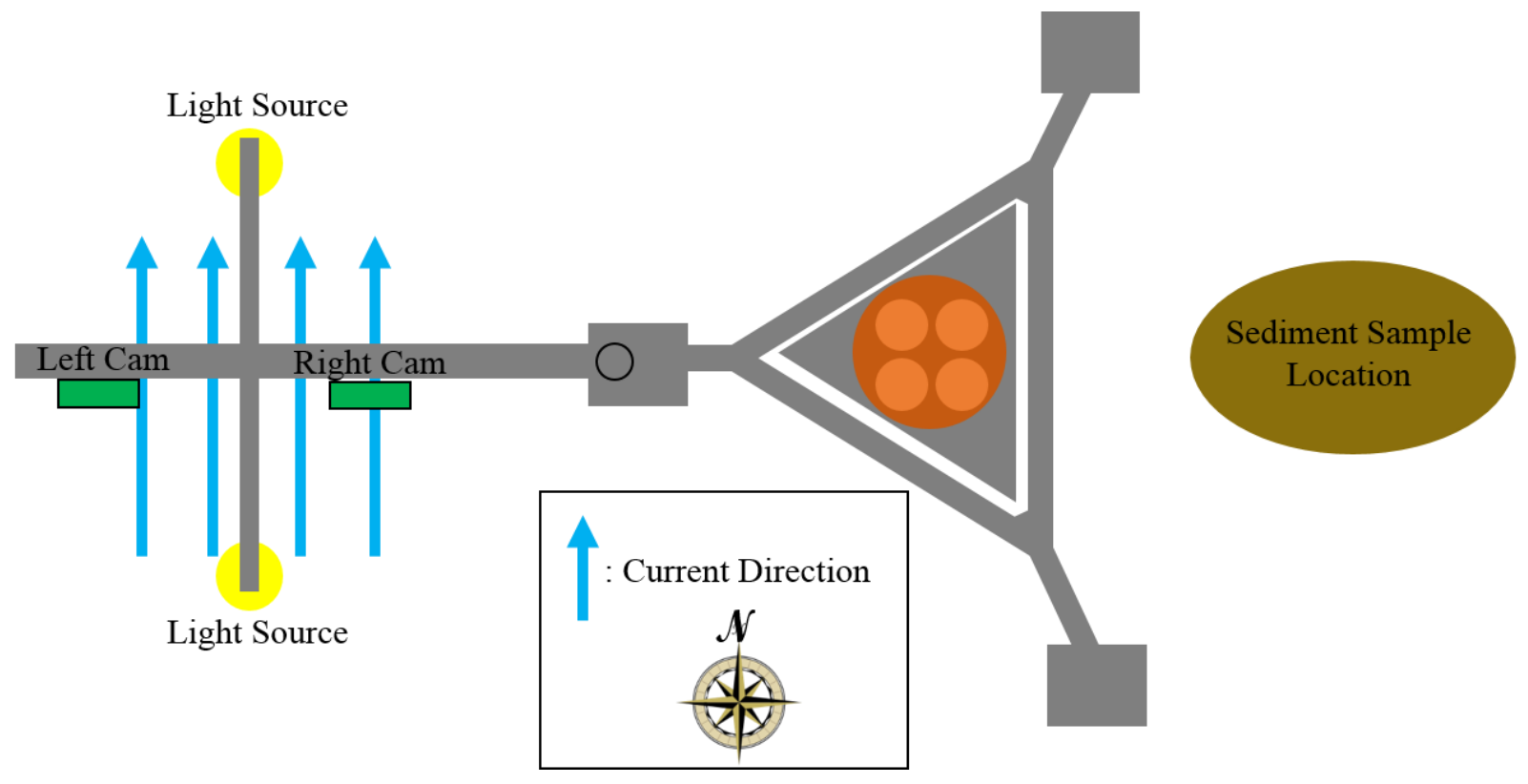

Figure 3-24. Top view illustration of tripod system describing sediment sample collection site.

Grain size analysis procedures were conducted both before and after shell hash was dissolved from each sample to quantitatively describe the shell hash content by means of grain size. Samples were processed twice to ensure repeatability following standard sieve and pipette procedures described by Folk [1980]. Shell hash content was dissolved from each sample by periodically adding $10 \% \mathrm{HCl}$ solution to the samples over a span of 4 days. Approximately 40 $\mathrm{ml}$ of $\mathrm{HCl}$ were added to each sample daily. Sample statistics for grain size distribution data were calculated using logarithmic Folk and Ward [1957] graphical measures implemented by GRADISTAT grain size analysis software [Blott and Pye, 2001].

Results from grain size analysis of the sediment are found in Table 3-3 and are consistent with findings from Ward and McAvoy [2014]. Sediment samples prior to $\mathrm{HCl}$ digestion were classified as moderately well sorted, slightly gravelly medium sand with high shell hash content. Similarly, samples post digestion were classified as moderately well sorted slightly gravelly medium sand. However, the total sample weight decreased from $17 \%-22 \%$ following digestion 
of shell hash, suggesting a high percentage of shell hash content. Major decreases in class weight percentage can be seen in phi sizes between -2 and $0.5(4 \mathrm{~mm}-0.707 \mathrm{~mm})$, implicating a majority of the shell hash content was present in these class sizes. An increase in weight at phi sizes $3,3.5$, and $4(0.125 \mathrm{~mm}, 0.088 \mathrm{~mm}, 0.0625 \mathrm{~mm})$ post digestion can be explained due to the expedited breakdown of the quartz sand upon exposure to the $10 \% \mathrm{HCl}$ solution. The addition of weight at these phi sizes was small compared to the overall weight of the phi classes of interest containing shell hash. These sets of statistics were not used in the determination of theoretical backscatter strength based on inclusion model scattering but calculated to give a general idea of the amount of shell hash content within each sample.

Table 3-3. Grain statistics from Portsmouth Harbor sediment samples utilizing Folk and Ward [1957] logarithmic method. Skewness and kurtosis are dimensionless parameters and mean and median grain size are in units of $\phi$, where $\phi=-\log _{2}(\mathrm{~mm})$

\begin{tabular}{|cccccc|}
\hline Sample ID & Mean, $\phi$ & Median, $\phi$ & Sorting, $\phi$ & Skewness & Kurtosis \\
\hline 1.A & $1.076(0.47 \mathrm{~mm})$ & 1.133 & 0.690 & -0.217 & 1.147 \\
\hline 1.B & $1.067(0.48 \mathrm{~mm})$ & 1.124 & 0.687 & -0.214 & 1.155 \\
\hline 1.A no shell & $1.206(0.43 \mathrm{~mm})$ & 1.215 & 0.517 & -0.060 & 0.977 \\
\hline 2.A no shell & $1.215(0.43 \mathrm{~mm})$ & 1.239 & 0.581 & -0.132 & 0.972 \\
\hline
\end{tabular}



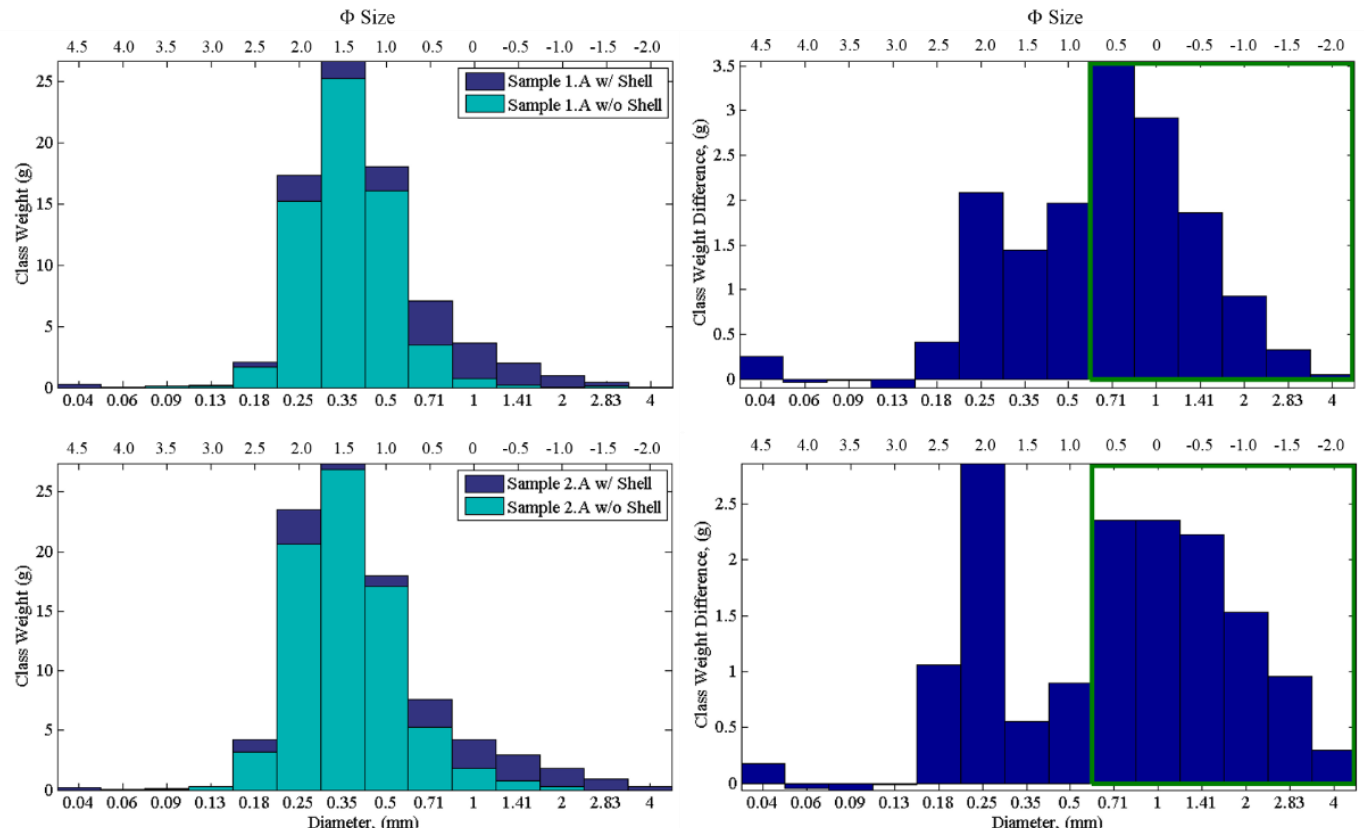

Figure 3-25. Grain size distribution by weight of sediment samples shown in left panel. Histograms on the right panel show difference in class weight between samples pre- and post- $\mathrm{HCl}$ digestion (total sample weight w/ shell total sample weight w/o shell). Therefore a positive reading in weight difference signifies higher concentration of shell hash at the respective grain size. The highlighted green boxes indicate grain sizes where shell hash was visible.

Grain size distribution statistics from Sample 1.A, with shell hash included, were used as input to the inclusion scattering model discussed in Chapter 2.2. The class weight distribution was converted to volume size distribution by Equation 14 allowing for calculation of the seabed backscattering coefficient by Equation 10 (Figure 3-26). 


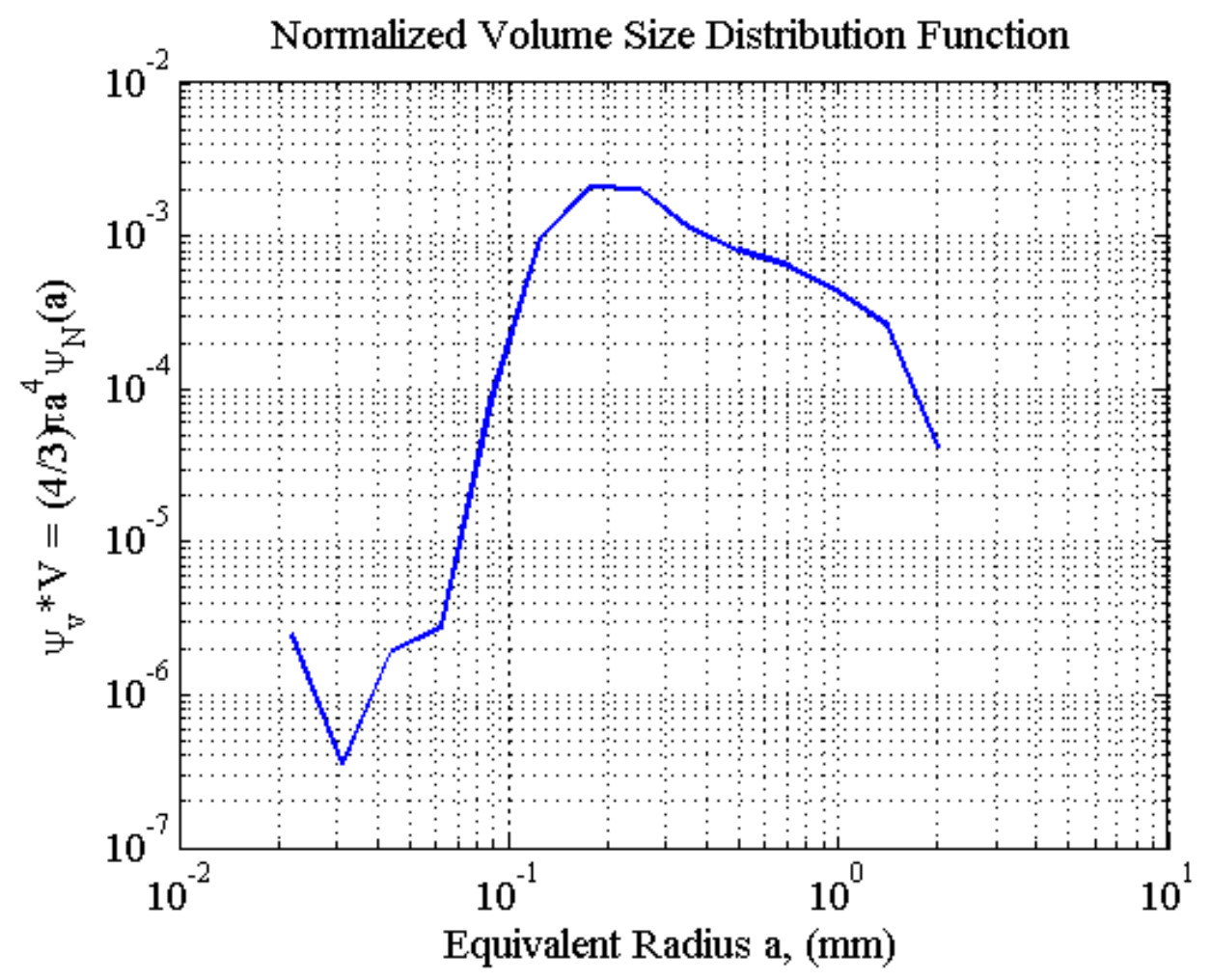

Figure 3-26. Normalized volume distribution function of sample 1.A.

The theoretical prediction of backscatter by the inclusion model assuming the volume size distribution curve in Figure 3-26, is shown in Figure 3-27. Acoustic parameters of the sediment, individual grains, and water, needed as input to the discrete inclusion scattering model, were taken as those described in Ivakin [2004] from the SAX99 site [Thorsos, 1999] and were as follows: $\rho=\frac{2 g}{\mathrm{~cm}^{3}}, c=1.7 \frac{\mathrm{km}}{\mathrm{s}}, \delta=0.01$ (sediments); $\rho_{\alpha}=\frac{2.7 \mathrm{~g}}{\mathrm{~cm}^{3}}, c_{\alpha}=5.7 \frac{\mathrm{km}}{\mathrm{s}}, \delta=0$ (individual grains); and $\rho_{w}=\frac{1 \mathrm{~g}}{\mathrm{~cm}^{3}}, c_{w}=1.5 \frac{\mathrm{km}}{\mathrm{s}}$ (water). The SAX99 site was comprised of similar sedimentary composition compared to the sand wave field as described by Richardson et al. [2001]. Both sites consisted of predominately moderately well-sorted quartz sand with the presence of shell hash. 


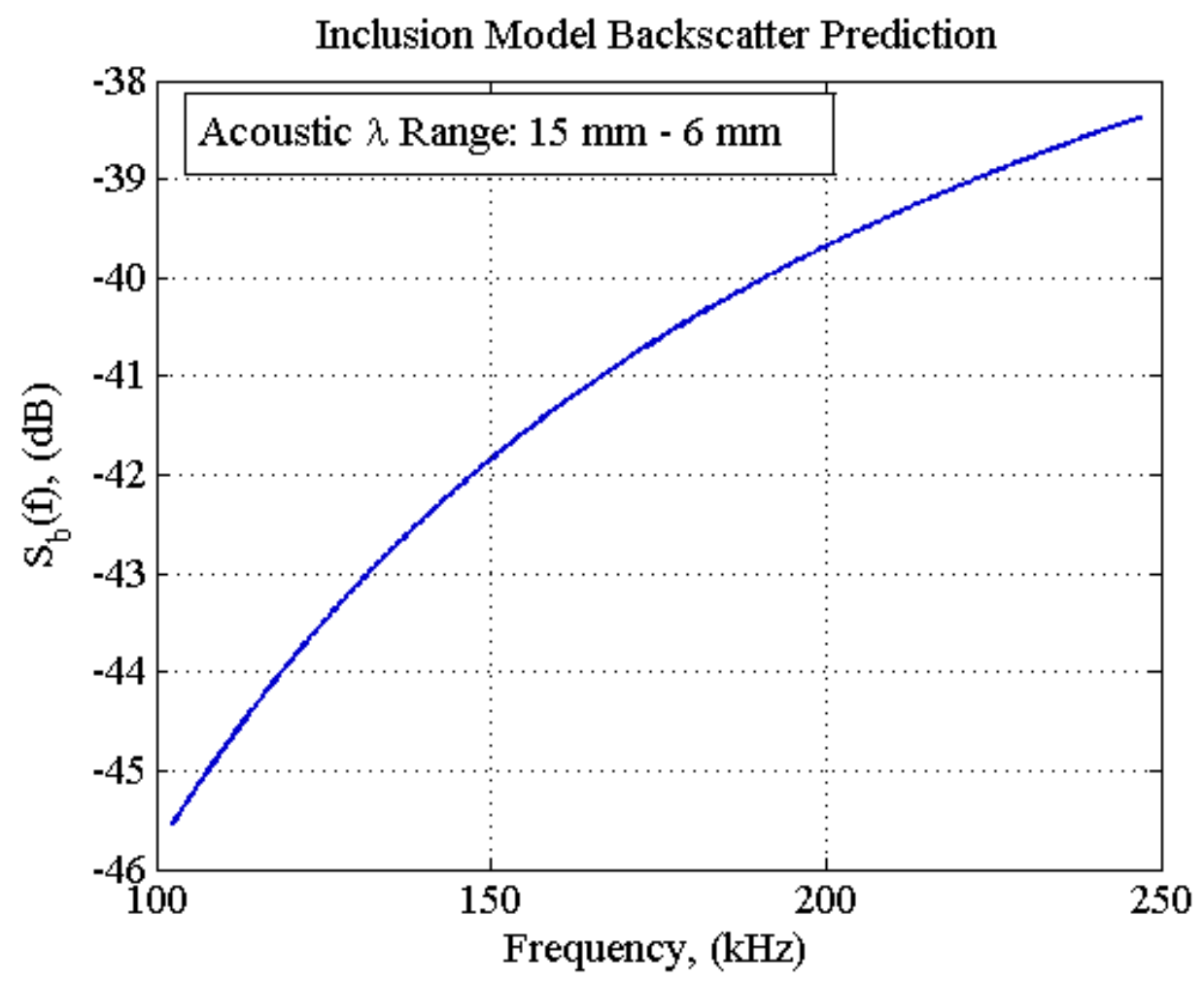

Figure 3-27. Inclusion model backscattering results. Values reported for $\mathrm{S}_{\mathrm{b}}$ on the $\mathrm{y}$-axis are insignificant due to normalization of $m_{s}$ based on lack of input parameters. The shape of the curve remains significant for analysis.

Absolute backscatter levels reported in Figure 3-27 were insignificant when comparing them to acoustic backscatter observations due to the offset in amplitude of $m_{s}$ based on the estimate of acoustic sediment input parameters utilized with Equation 10. Estimation of such parameters still provided an accurate re-creation of the theoretical backscatter prediction with respect to frequency and was only offset in amplitude. Figure 3-28 shows an example of an offset in only amplitude caused by alteration of sound speed and density of the sediment volume and individual grains. 


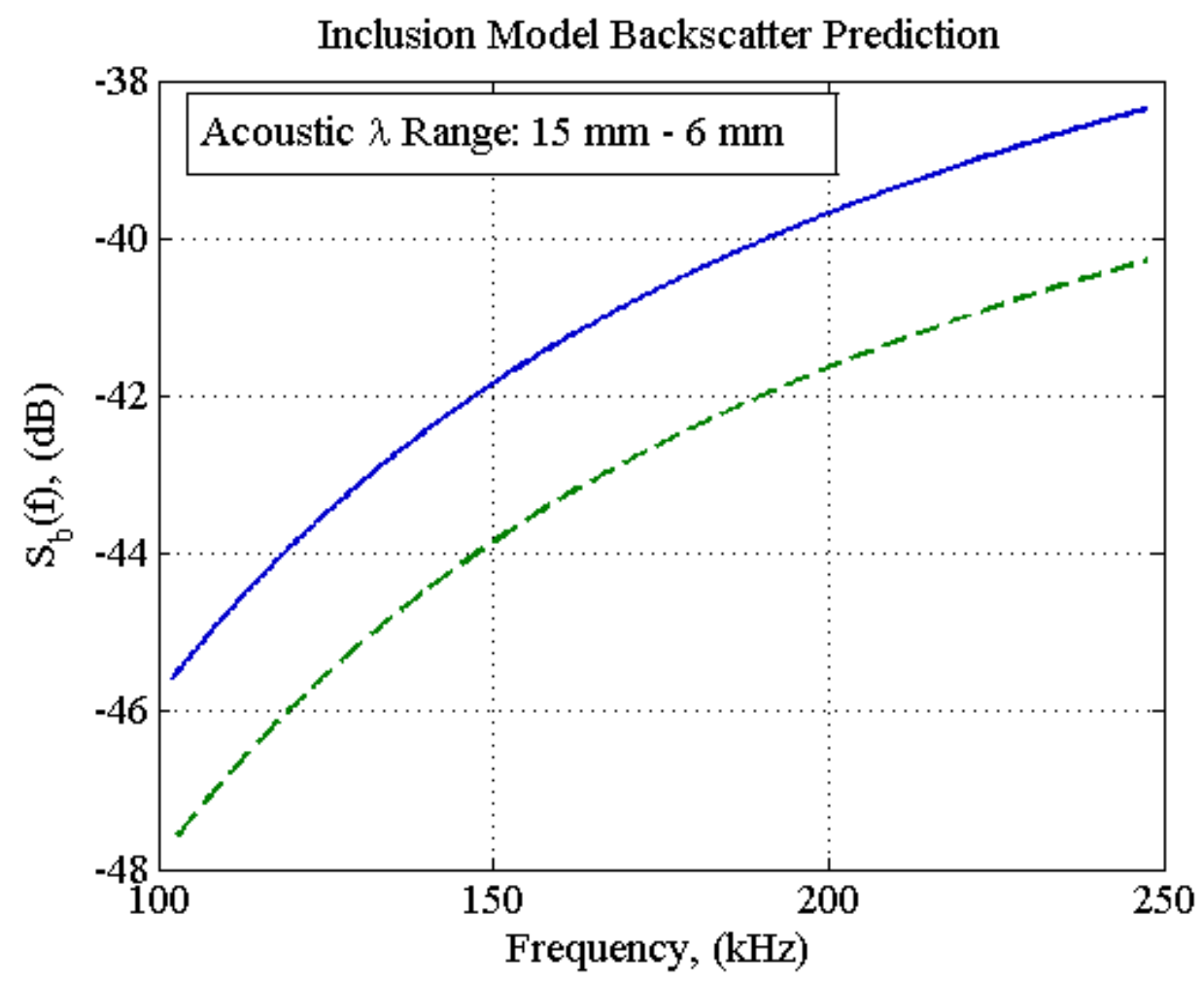

Figure 3-28. Backscatter predictions from inclusion model showing magnitude shift due to variation in sediment acoustic properties, density and sound speed. The shift in amplitude between the two curves is due to an increase of sediment and particle densities of $500 \mathrm{~kg} / \mathrm{m}^{3}$ and sound speeds of $500 \mathrm{~m} / \mathrm{s}$, respectively.

The slope of the curve remains of significance in comparison to acoustic backscatter observations and is ultimately controlled by the volume size distribution function. The presence of discrete inclusions, such as shells and coarse sand particles, are the critical scatterers within the distribution which control the shape of the backscatter response due to their relative size to the corresponding wavelengths of acoustic operating frequencies [Ivakin, 2004].

Evolution of scattering strength as predicted by the discrete inclusion scattering model from various volume size distribution functions is shown in Figure 3-29. As larger particle diameter sizes are removed from the volume size distribution, the magnitude of the slope evaluated at both frequency bands converges. Convergence of slope occurred for both frequency bands after the volume size distribution function was reduced to grain sizes between $1.4 \mathrm{~mm}$ to 
$0.25 \mathrm{~mm}$. This behavior suggests that the larger particles of the grain size distribution control the response of the discrete inclusion scattering model. As particles larger than $1.4 \mathrm{~mm}$ are added to the volume size distribution, the magnitude of the slope related to the response of the discrete inclusion scattering model begins to diverge.
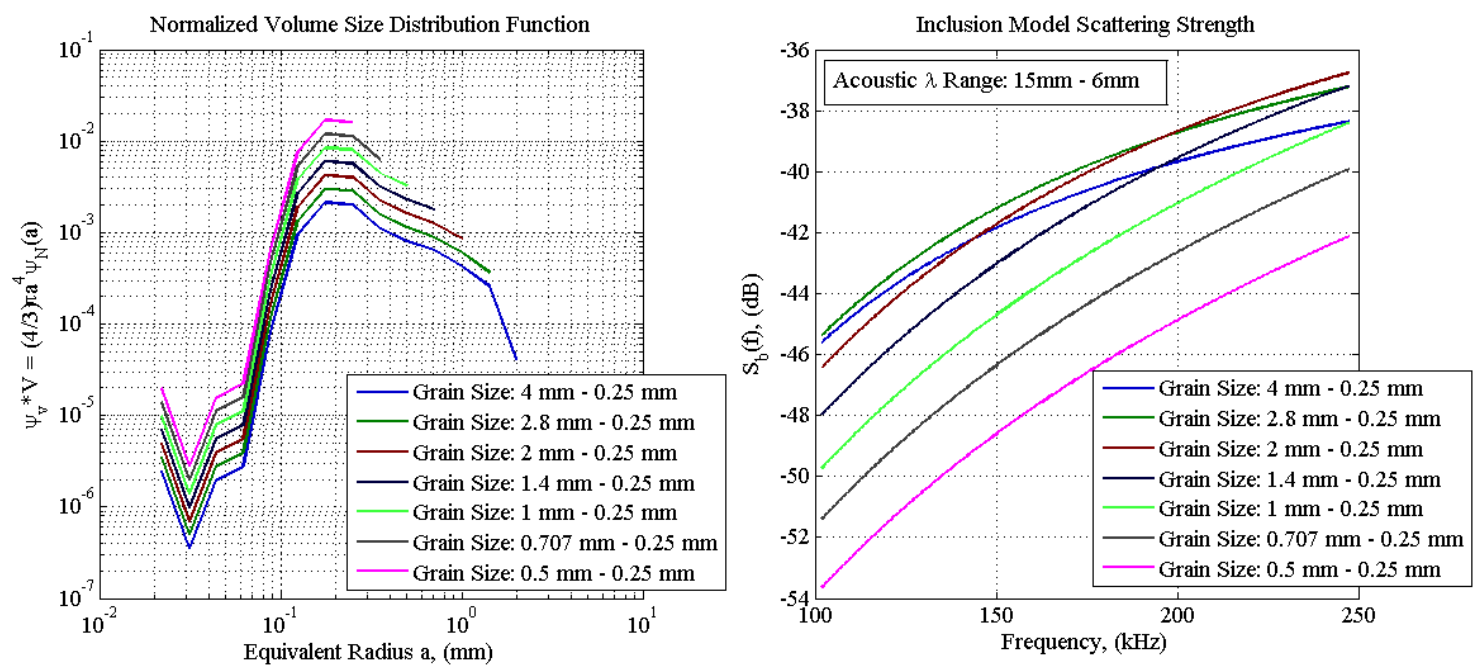

Figure 3-29. Comparison of predicted scattering strength of inclusion model with variable volume size distribution.

To make comparisons to acoustic backscatter data collected during the SBES survey, the slope of the theoretical backscatter curve produced from the discrete inclusion model, utilizing the full volume size distribution indicated by the dark blue line in Figure 3-29, was evaluated at the upper and lower frequency bands where acoustic data was collected. Resultant slopes estimated at the two frequency bands were evaluated with a polynomial fit in log-log space to develop a relationship between the expected rate of increase in backscatter with increasing frequency. This analysis suggested backscatter would increase proportional to $10 \log _{10} f^{2.21}$ for the lower frequency band and $10 \log _{10} f^{1.49}$ for the upper frequency band. Graphical results of the theoretical backscatter response and the linear regression of the upper and lower frequency bands is shown in Figure 3-30. 


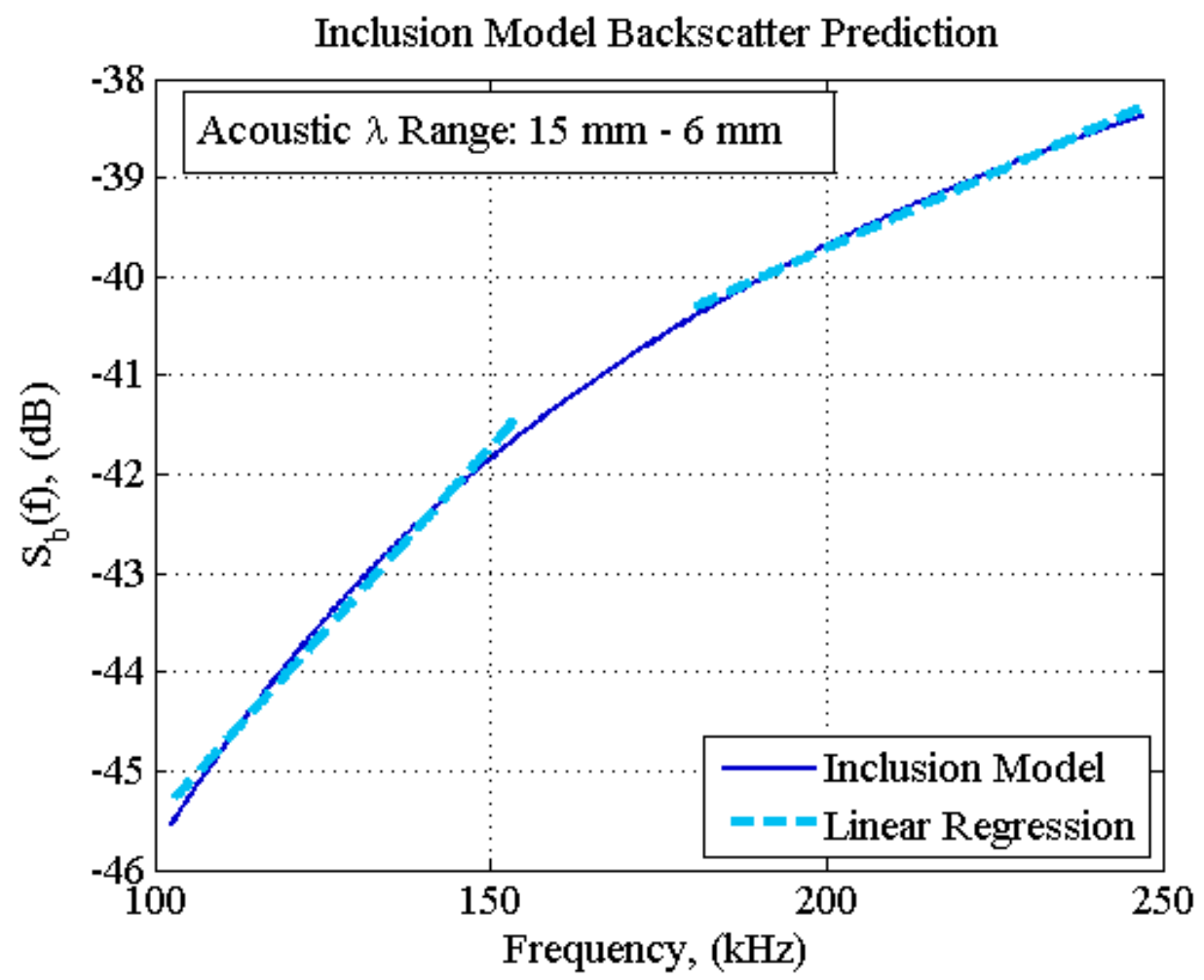

Figure 3-30. Inclusion model scattering backscatter response with line of best fit on upper and lower frequency bands. 


\section{CHAPTER 4}

\section{ACOUSTIC DATA COLLECTION AND RESULTS}

\subsection{Logistics of Acoustic Measurements}

A SBES survey was conducted between the hours of 6:34 PM UTC and 10:14 PM UTC on 1 October 2014. Seafloor backscatter measurements were collected at a grazing angle of $45^{\circ}$ perpendicular to the direction of vessel travel. Two unique Kongsberg SBES models, an ES120C and an ES200-CD, were utilized in collection of seafloor backscatter. The ES120-C and ES200-CD were circular 'piston' transducers each with a 1-way beamwidth of $7^{\circ}$, evenly divided into four quadrants. Each transducer was interfaced to a prototype Simrad wideband transceiver (WBT), allowing for collection of broadband seafloor backscatter. A $2.05 \mathrm{~ms}$ linear frequencymoduluated (LFM) pulse was utilized for each transducers' transmit signal. The transmit signal associated with the ES120-C model covered a frequency range of frequencies from $95-160 \mathrm{kHz}$, while the signal associated with the ES200-CD model covered a frequency range of 160-260 kHz. Frequencies from $\sim 100 \mathrm{kHz}-150 \mathrm{kHz}$ and $\sim 170 \mathrm{kHz}-250 \mathrm{kHz}$ were analyzed for this work. The range of useable frequency per each transducer was limited by distortion at the ends of each broadband signal.

\subsection{Acoustic Processing Methods}

Acoustic processing methods were separated into two different processes, calibration and field processing, with the addition of common split-beam processing techniques applied to each method. The results from the calibration process were used to apply to the field data to account 
for any offset in measurements observed by either SBES system. Split-beam processing techniques were applied to each acoustic processing method to accurately determine the location of respective targets within the beam of the transducers.

\section{Split-Beam Processing}

For any given return signal from a target within the water column, the location of that target within the beam of the transducer must be accounted for to truly determine the strength of the return signal. If a desired discrete target is off the center of the beam i.e. the beam's maximum response axis (MRA), the acoustic response from the target will appear weaker because less sound is returned to the transducer when outside of this axis. The same applies to an extended target, such as the seafloor, where only part of the returned seafloor response is coincident with the transducer's MRA. Therefore, determination of the along-track and acrosstrack phase angles associated with a return signal is required to either predict a target's angle within the beam of the transducer or determine the section of a return signal located on the MRA. Burdic [1991] provides split-aperture processing techniques which can be directly applied to either situation.

Four separate quadrants within each SBES system are recorded separately during data acquisition, and summed coherently to form the receive signal described in Equation 23. The electrical phase difference between pairs of the quadrants can then be used to estimate the angle. Pairs of quadrants are defined by the transducers' roll and pitch planes. Utilizing the match filtered outputs of Equation 23, which are defined as $s_{m f, i}(t)=s_{i}(t) \otimes s_{o}^{*}(-t)$, where the subscript $i$ represents quadrants one through four of the transducers. The electrical phase angles for each system can then be computed as, 


$$
\begin{array}{ll}
\psi_{\text {along }}=\tan ^{-1} \frac{\operatorname{Im}\left\{\left(s_{m f, 1}+s_{m f, 2}\right)\left(s_{m f, 3}+s_{m f, 4}\right)^{*}\right\}}{\operatorname{Re}\left\{\left(s_{m f, 1}+s_{m f, 2}\right)\left(s_{m f, 3}+s_{m f, 4}\right)^{*}\right\}}, & \text { Equation } 19 \\
\psi_{\text {across }}=\tan ^{-1} \frac{\operatorname{Im}\left\{\left(s_{m f, 1}+s_{m f, 4}\right)\left(s_{m f, 2}+s_{m f, 3}\right)^{*}\right\}}{\operatorname{Re}\left\{\left(s_{m f, 1}+s_{m f, 4}\right)\left(s_{m f, 2}+s_{m f, 3}\right)^{*}\right\}} & \text { Equation } 20
\end{array}
$$

The combined phase angle is then calculated by,

$$
\psi=\sqrt{\psi_{\text {along }}^{2}+\psi_{\text {across }}^{2}} .
$$

The combined phase angle was used to sort the calibration data necessary to determine the overall calibration coefficient as a function of frequency for each SBES system. The across ship phase angle was used in bottom detect methods associated with the acoustic filed data discussed later in this chapters.

\section{$\underline{\text { Scattering from a Single Discrete Target: Sonar Calibration }}$}

Prior to use in the field, each SBES system was calibrated aboard the UNH R/V Coastal Surveyor utilizing the same transducer mount and, WBT configuration used during collection of the field data. The objective of the at-sea calibration was to understand the frequency response of each transducer system necessary to estimate target strength (TS) of the seafloor, which is then converted to seafloor backscattering strength, $S_{b}$. The calibration process consisted of swinging a $38.1 \mathrm{~mm}$ tungsten carbide sphere through each systems' four quadrants while recording data for a standard sphere calibration as described by Foote, et al [1987]. The scattered pressure response, $p_{s}$ from the sphere, received by a transducer is treated as the response from a single discrete target at a range $r$, as a function of time, $t$. This can be written as the convolution of the ideal transmit signal, $s_{o}$, the impulse response from the transmit 
transducer, $h_{t r x}$, the impulse response of the medium of travel, $h_{p}$, and the impulse response of the discrete target, $h_{t r g}$;

$$
p_{s}(t)=s_{o}\left(t-t_{o}\right) \otimes h_{t r x} \otimes h_{p} \otimes h_{t r g}
$$

where $t_{o}$ is the arrival time of the transmit signal from the target [Weber, 2015]. The scattered pressure response from Equation 22 is then received by a receive transducer and converted to a receive signal by the additional convolution of the impulse response from the receive transducer, $h_{t r r}$

$$
s(t)=s_{o}\left(t-t_{o}\right) \otimes h_{t r x} \otimes h_{p} \otimes h_{t r g} \otimes h_{t r r}
$$

Equation 23

The convolution of $h_{t r x}$ and $h_{t r r}$ can be combined to $h_{t r}=h_{t r x} \otimes h_{t r r}$ due to the fact that the receive and transmit transducer are the same. Because of the broadband frequency nature of this work, the Fourier transform of Equation 23, is computed and described as Equation 24,

$$
S(f)=\int_{-T / 2}^{T / 2} S(t) e^{-j 2 \pi f t} d t=S_{o}(f) H_{t r} \frac{e^{-2 a r}}{r^{2}} H_{t r g}
$$

where $S_{o}(f)$ is the Fourier transform of the ideal transmit signal, $H_{t r}$ is the Fourier transform of the combined impulse responses of the transmit and receive transducer, and $H_{t r g}$ is the Fourier transform of the impulse response of the target. The limits of the integral in Equation 24 are equal to the length of $s_{o}(t)$, in time, $T$. Integration over the entire length of the transmit signal ensures that the analysis covers the complete scattered response of the target. Propagation losses due to spherical spreading and absorption are calculated by $\frac{e^{-2 a r}}{r^{2}}$, where $a$ is the absorption coefficient in nepers/m and $r$ is the range to the target in meters. 
The frequency dependent backscattering cross section of a discrete, deterministic target is defined by,

$$
\sigma_{b s}(f)=\left|H_{t r g}(f)\right|^{2}
$$

Equation 25

Together, the measured response, $S(f)$, of a target with a known frequency dependent backscatter response, $\sigma_{b s}(f)$, can then be used to calculate a calibration offset for the system;

$$
C(f)=\frac{|S(f)|^{2}}{\sigma_{b s}(f)} \frac{r^{4}}{e^{-4 a r}}
$$

To increase the signal to noise ratio of the received signal, a match filter can be applied utilizing the ideal transmit signal, $s_{o}$. This is done by multiplying Equation 24 by the complex conjugate of the Fourier transform of the ideal signal, $S_{o}^{*}(f)$,

$$
S_{m f}(f)=S_{o}(f) S_{o}^{*}(f) H_{t r} \frac{e^{-2 a r}}{r^{2}} H_{t r g}
$$

Equation 27

The frequency dependent calibration factor then becomes,

$$
C(f)=\frac{\left|S_{m f}(f)\right|^{2}}{\sigma_{b s}(f)} \frac{r^{4}}{e^{-4 a r}}
$$

Equation 28 is then sorted as a function of combined phase angle, $\psi$, and fit to a $3^{\text {rd }}$ order polynomial. Phase angles were normalized by $\psi_{\text {norm }}=\psi f_{c} /_{f}$, where $f_{c}$ is the center frequency of the system, and $f$ is the frequency. This normalization was made under the assumption that the beamwidth was inversely proportional to frequency. The $3^{\text {rd }}$ degree polynomial fit was applied to the data for $\psi_{\text {norm }} \leq 1.6$ radians. The resultant $3^{\text {rd }}$ order coefficient, that represents the intercept of the polynomial fit at $\psi=0$, was applied as to the field data as the frequency dependent calibration coefficient. A return signal associated with a 
phase angle equal to zero corresponds to the section of the return on the transducer's MRA. The offset related to each SBES is shown in Figure 4-1. The difference between the two curves, the dashed being the modeled response of the sphere and the solid being the raw sonar response from the sphere, represents the calibration offset.
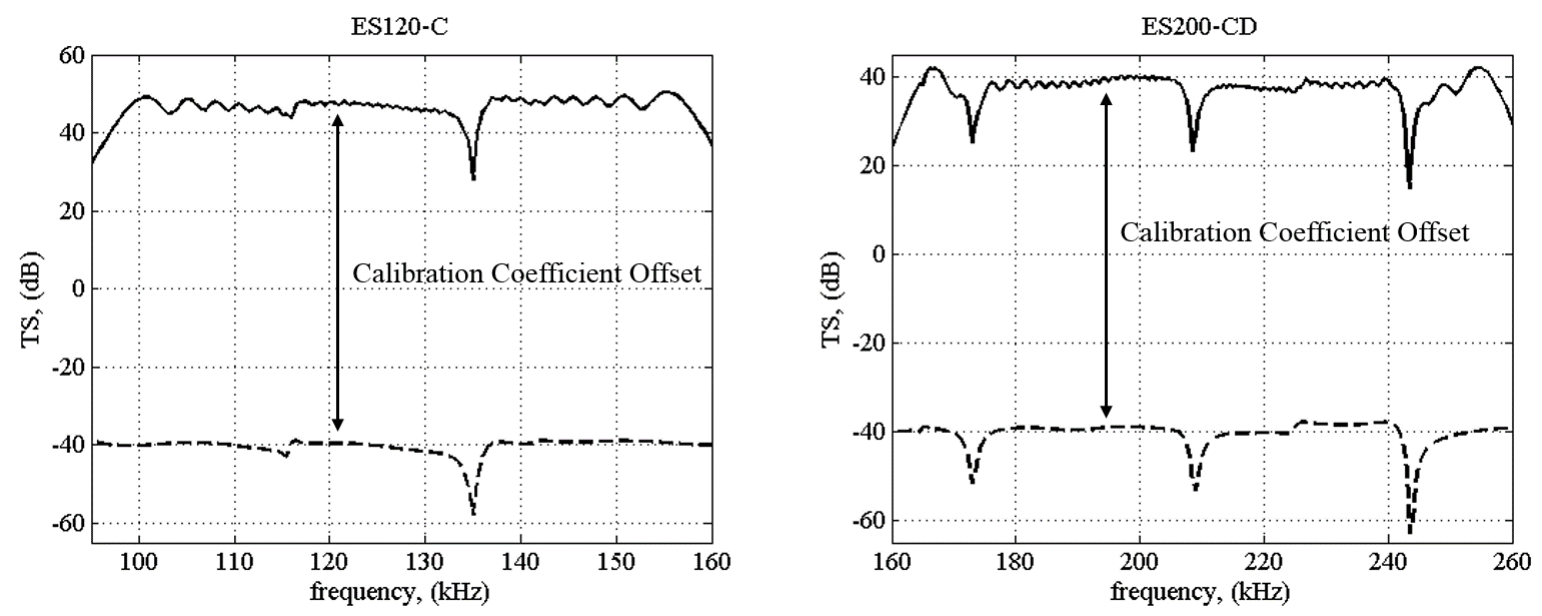

Figure 4-1. Results of $3^{\text {rd }}$ order polynomial fit to $38.1 \mathrm{~mm}$ tungsten carbide calibration sphere (solid black lines). The dashed lines are the estimated target strength of the $38.1 \mathrm{~mm}$ tungsten carbide calibration sphere.

\section{$\underline{\text { Scattering from an Extended Target: Seafloor Response }}$}

When examining the response of the seafloor received by the transducer, it is important to note that the response is now considered random. Equation 22 directly applies to this scenario with the exception that $h_{t r g}$ is no longer the impulse response of a single deterministic target, but a random extended target. The received scattered pressure from an extended target is recorded identical to that of a discrete target signal, but post processing techniques are altered to account for the random extended nature of the target [Weber, 2015].

When applying the Fourier transform to the received signal, similar to Equation 24, the scattered pressure must first be multiplied by a window function, $w(t)$. The window function is non-zero over the time duration for which the received scattered pressure will be analyzed. The 
duration of the window function can be found by dividing the length of the receive signal by the sampling frequency. Applying this in the frequency domain results in the convolution of the extended target version of Equation 24 with the Fourier transform of the window function $W(f)$,

$$
S(f)=S_{o}(f) H_{t r} \frac{e^{-2 a r}}{r^{2}} H_{t r g} \otimes W(f)
$$

Equation 29

According to Harris [1978], the addition of the convolution term can be treated as the incoherent processing gain for an extended target, such as the seafloor. It is equivalent to multiplying by $\sqrt{T}$, where $T$ remains the length of the ideal signal in seconds. Equation 29 can then be written as,

$$
\left\langle|S(f)|^{2}\right\rangle=C(f) \frac{e^{-4 a r}}{r^{4}}\left\langle\left|H_{t r g}(f)\right|^{2}\right\rangle T,
$$

Equation 30

where the brackets \langle\rangle indicate an ensemble average, and $\left\langle\left|H_{\operatorname{trg}}(f)\right|^{2}\right\rangle T$ is the target strength (TS) of the seafloor. TS is computed by the product of the backscattering cross section, $\sigma(f)$, and the ensonified area, $A$. Equation 30 is then re-written as Equation 31,

$$
\sigma(f)=\frac{\left\langle\left|H_{t r g}(f)\right|^{2}\right\rangle T}{A}=\frac{\left\langle|S(f)|^{2}\right\rangle}{C(f)} \frac{r^{4}}{e^{-4 a r}} \frac{2 \sin \theta_{i}}{c T \theta_{e q} r}
$$

where $\theta_{i}$ is the grazing angle, $c$ is the sound speed in $\mathrm{m} / \mathrm{s}$, and $\theta_{e q}$ is the equivalent beamwidth. The ensonified area $A$, which applies to measurements taken at grazing angles beyond normal incidence, is quantified as a rectangular area of the seafloor encompassed by the beam, where $\theta_{e q} r$ accounts for one side of the rectangle and $\frac{c T}{2 \sin \theta_{i}}$ accounts for the other [Lurton, 2010]. Similar to the previous section, a matched filter can also be applied to the receive signal in which Equation 31 becomes, 


$$
\sigma(f)=\frac{\left\langle\left|S_{m f}(f)\right|^{2}\right\rangle}{C(f)} \frac{r^{4}}{e^{-4 a r}} \frac{2 \sin \theta_{i}}{c T \theta_{e q} r}
$$

Equation 32 is converted to backscatter strength in units of decibels by Equation 33,

$$
S_{b}(f)=10 \log _{10}(\sigma(f)) .
$$

Equation 33

\subsection{Observations of Seafloor Backscatter}

Backscatter observations were collected over the sand wave field and gravel field following the survey transect outlined in Figure 4-2.

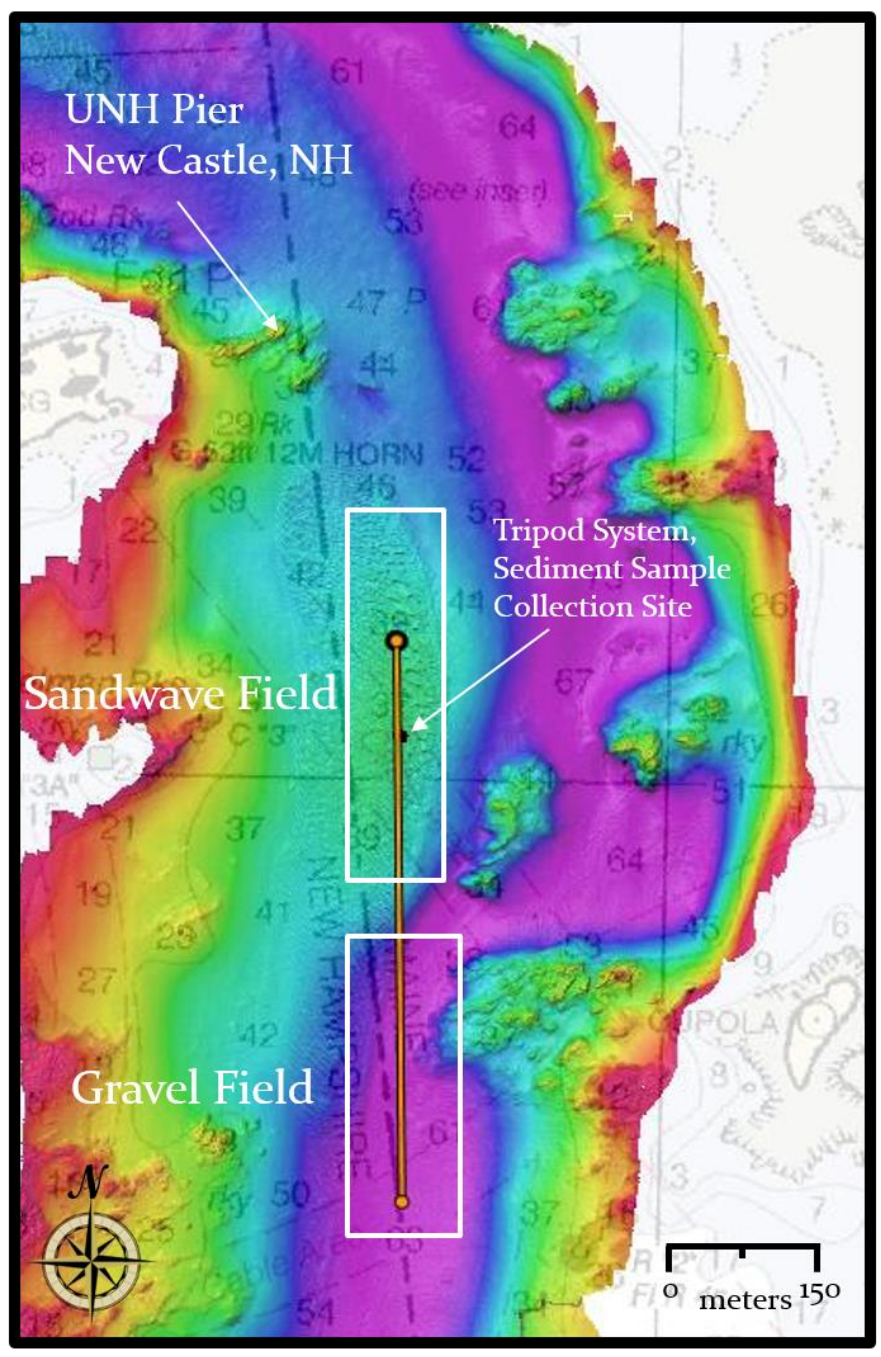

Figure 4-2. Survey transect through sand wave and gravel field. 
Both SBES systems operated at a ping rate of 1 ping per second. Over the 4-hour survey period, coincident with a flood tide event, the vessel made a total of 43 passes over the sand wave and gravel fields.

\section{Sand Wave Field}

Data collected over a $\sim 100 \mathrm{~m}$ stretch along the survey line, centered over the location of the tripod system (50 m stretch on either side of the tripod), were used to evaluate the response of the seafloor as a function of time and frequency. Each $100 \mathrm{~m}$ pass contained approximately 30 pings from each SBES system. The seafloor was assumed to be relatively uniform over this section of the transect by inspection of bathymetry data provided by Felzenburg [2009]. Verification of this assumption was done by calculating the average scattering strength of $25 \mathrm{~m}$ sections along the $100 \mathrm{~m}$ stretch (Figure 4-3). 


\section{Average Bottom Backscattering Strength}

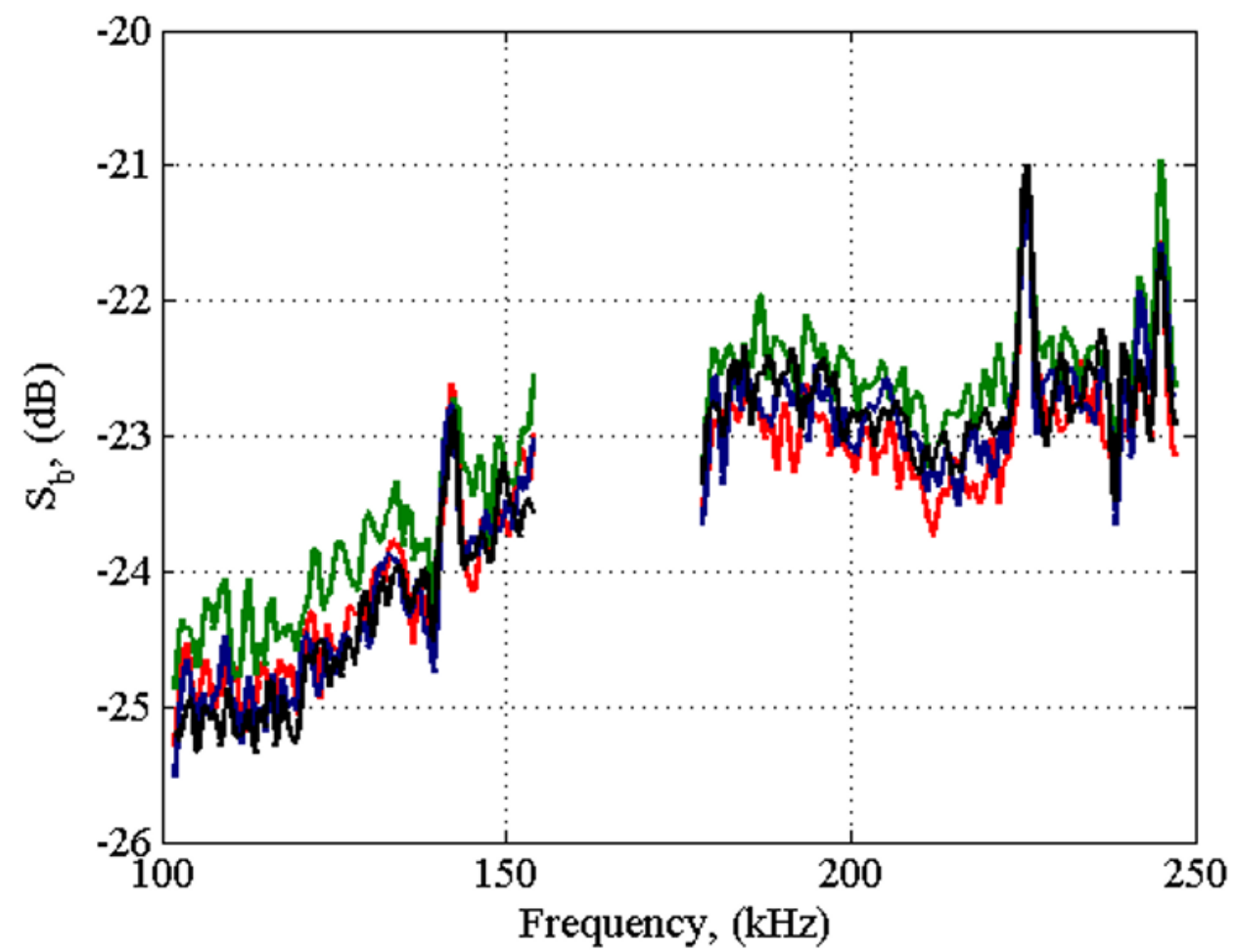

Figure 4-3. Stability check of acoustic data across the $100 \mathrm{~m}$ transect centered about the tripod. Each colored line represents a $25 \mathrm{~m}$ section of the $100 \mathrm{~m}$ line.

Results from each $25 \mathrm{~m}$ section proved to be nearly identical, verifying the assumption that the seafloor was relatively uniform in the sense of an acoustic backscatter response over the $100 \mathrm{~m}$ stretch.

In calculating the backscatter response in Figure 4-3 as well as subsequent results, amplitude and phase bottom detect methods were used for each data ping of the raw match filtered output to determine the section of the received signal needed to calculate the bottom backscatter strength along each transducers' MRA. A maximum amplitude detect was first performed to locate the general position of the seabed return. A zero-crossing phase detect described by Lurton [2010] was then used to accurately determine the exact range to the seabed coincident with the transducers' MRA, needed to calculate $S_{b}(f)$. In reference to Figure 4-4, the 
top plot represents one ping of the raw match filtered output needed for a maximum amplitude detect. The portion of the signal outlined in black identifies the area of the signal used to locate the local maximum, which is assumed to be the return from the seabed. The middle plot represents the across ship phase angle. The magenta dot marks the location of the zero crossing point and the location of the seabed within the received signal along the MRA. The bottom plot is the corresponding along ship phase angle.
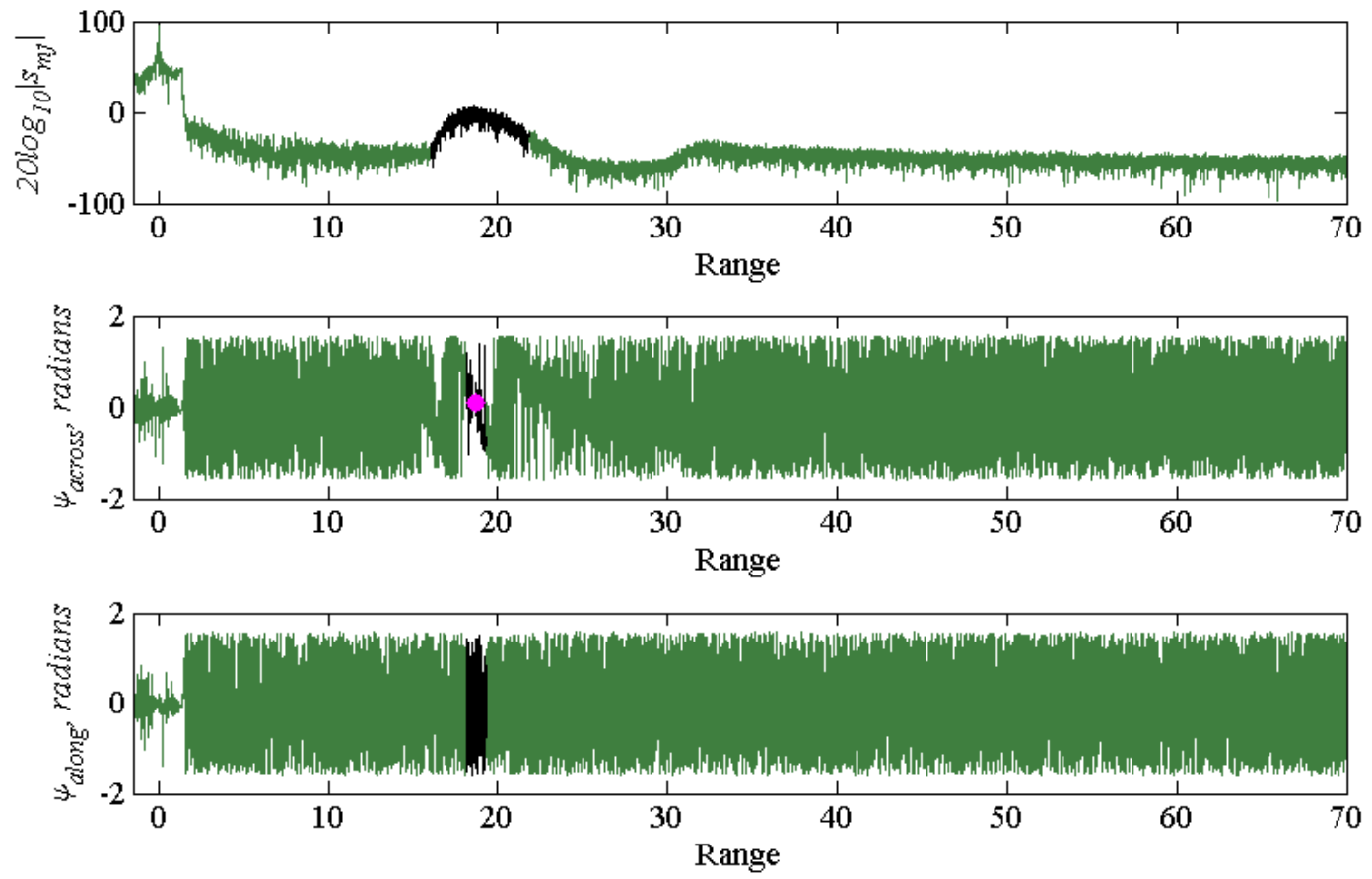

Figure 4-4. Graphical representation of the bottom detect methods used in determining the range to the seabed and section of received signal coincident on the MRA.

Figure 4-5 presents example results of bottom detects over a small section of the survey line. The magenta dots common to each plot mark the seafloor coincident with the MRA of the transducer in the top plot and a zero-value across ship phase angle in the bottom plot. 


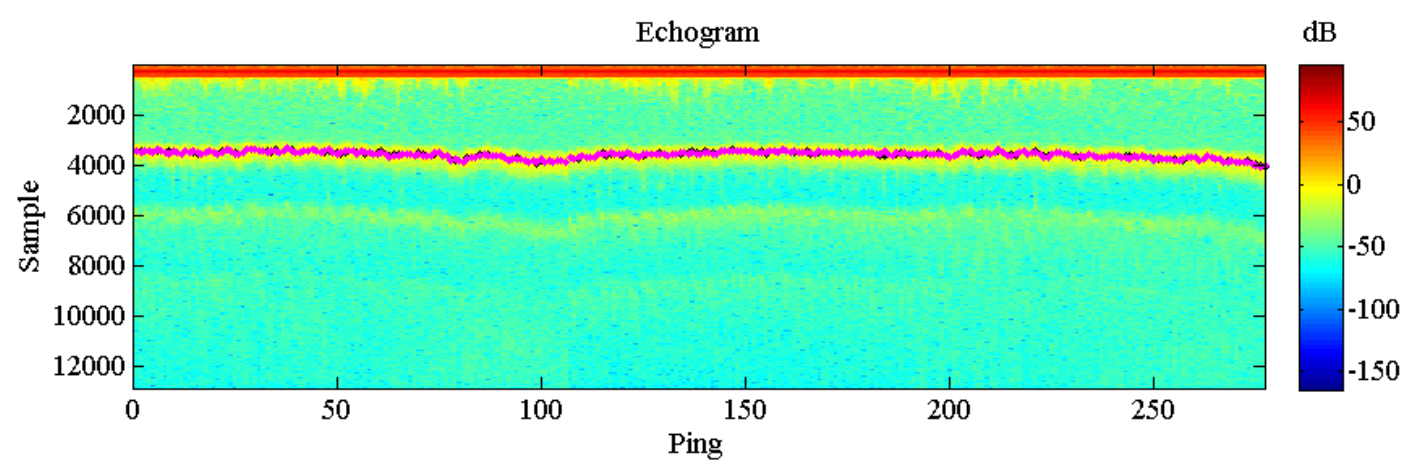

Across Ship Phase Angle

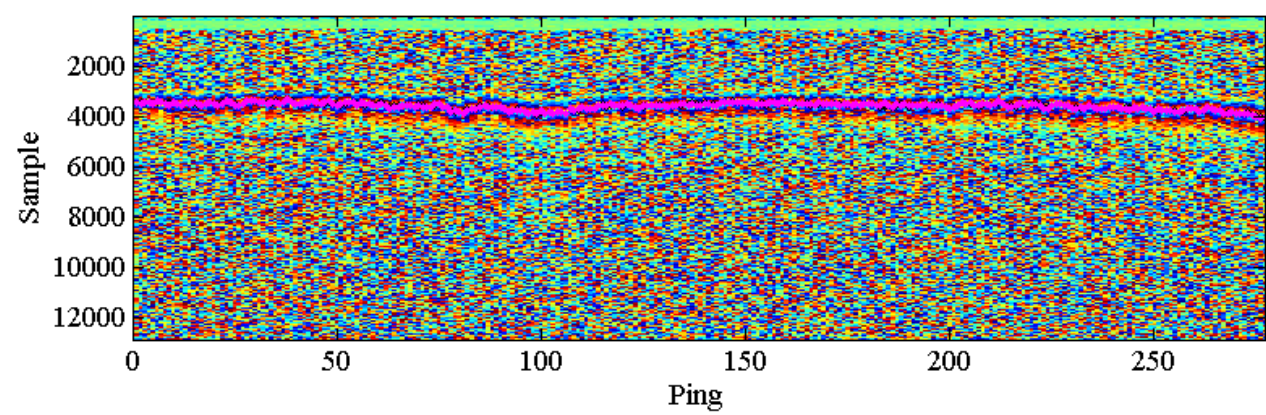

Figure 4-5. Echogram and across ship phase angle response over a small section of seafloor from the acoustic survey. Magenta dots represent phase angle bottom detect results outlining the seafloor in the top plot and $\psi_{\text {across }}=$ 0 in the bottom plot.

Seafloor backscattering strength was then calculated using Equation 32 and Equation 33 and its amplitude was evaluated as a function of time to analyze the stability of the backscatter response over the survey period. The stability of the measurements was analyzed because of the dynamic nature of the survey environment. Sediment transport analysis indicated incipient motion of the sediment occurred during periods of high current velocity. A single frequency from both upper and lower frequency bands was examined for temporal stability.

Figure 4-6 shows that the backscatter was relatively stable over the course of the survey period for both upper and lower frequency bands. At either frequency, the observed scattering strength varied minimally by only $1 \mathrm{~dB}-2 \mathrm{~dB}$. 

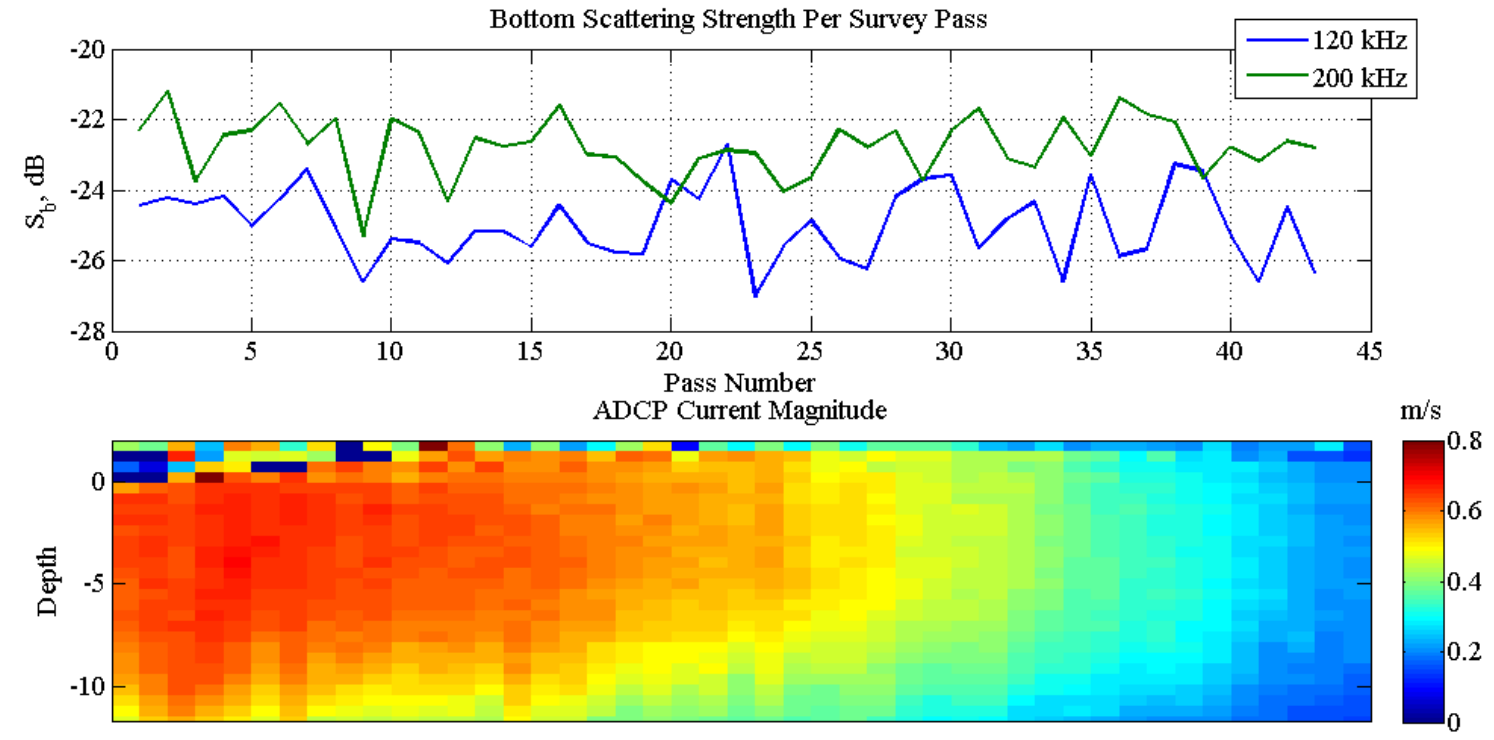

Figure 4-6. Backscatter data as a function of time over the sand wave field showing stability of scattering strength throughout the duration of the survey. Magnitude of the current is shown in the bottom plot to emphasize the dynamic environment of the survey area.

Stability in the backscatter response throughout the survey period mirrored that of the roughness spectrum discussed in Chapter 3.2. Therefore, backscatter was averaged in time, over all survey passes and analyzed as only a function of frequency (Figure 4-7). At the lower frequencies, the scattering strength increases proportional to frequency according to $10 \log _{10} f^{1.03}$. At the upper frequency band, this relationship changes to an increase in scattering strength proportional to $10 \log _{10} f^{0.33}$. The decrease in the slope of the backscatter response by frequency occurs between the gap in data and suggests a change in the seafloor scattering mechanism controlling the scattering. 


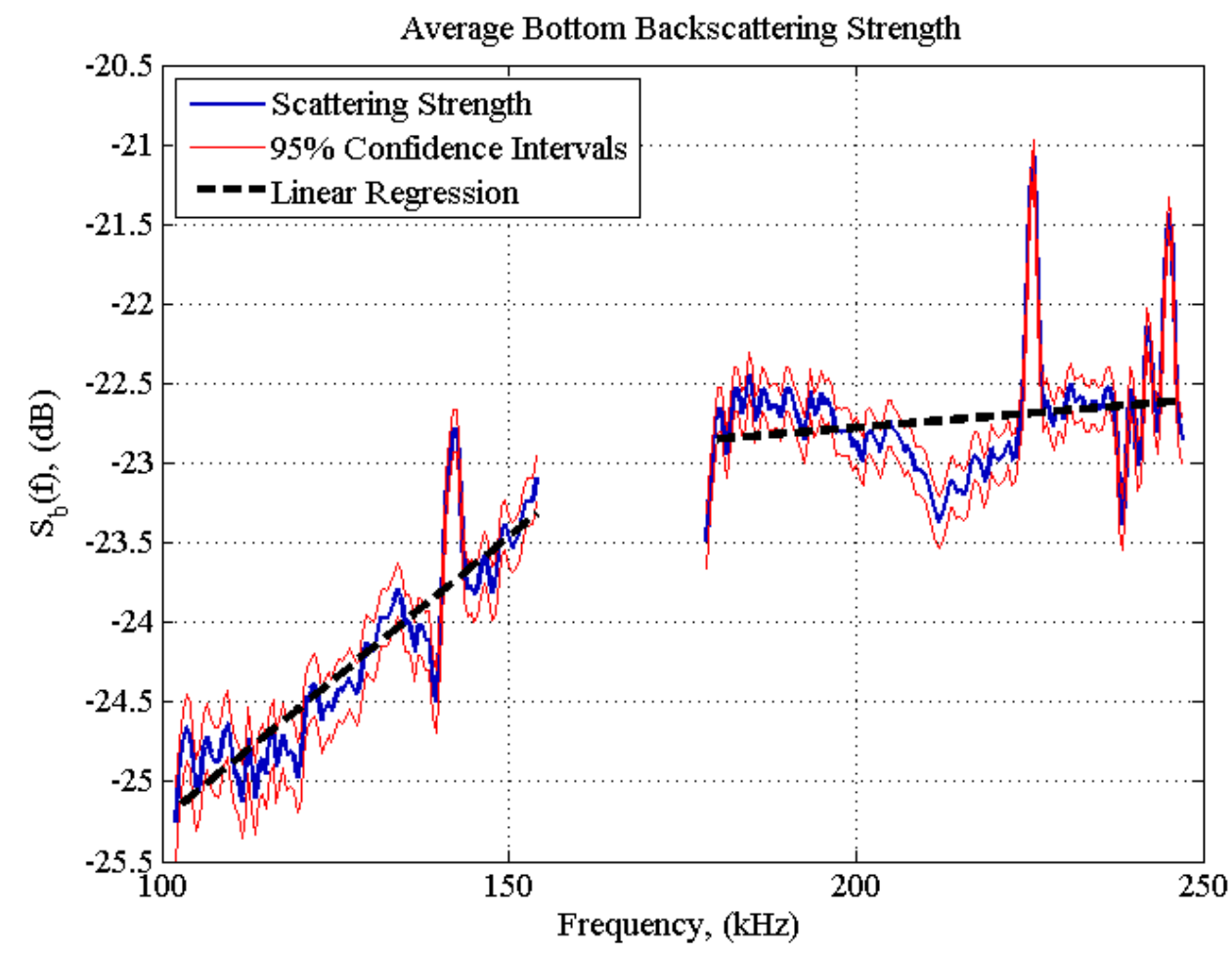

Figure 4-7. Average bottom backscattering strength over each survey pass as a function of frequency. Dashed black lines indicate the linear regression of each frequency band.

In comparison to both scattering models described in Chapter 2.1, the acoustic observations showed no relationship to either presumed scattering mechanism. The linear regression of each theoretical prediction of backscatter overlaid on the acoustic data from the scattering model results outlined in Chapter 3.2 revealed that neither model was a good descriptor of the backscatter response observed over the sand wave field (Figure 4-8). 


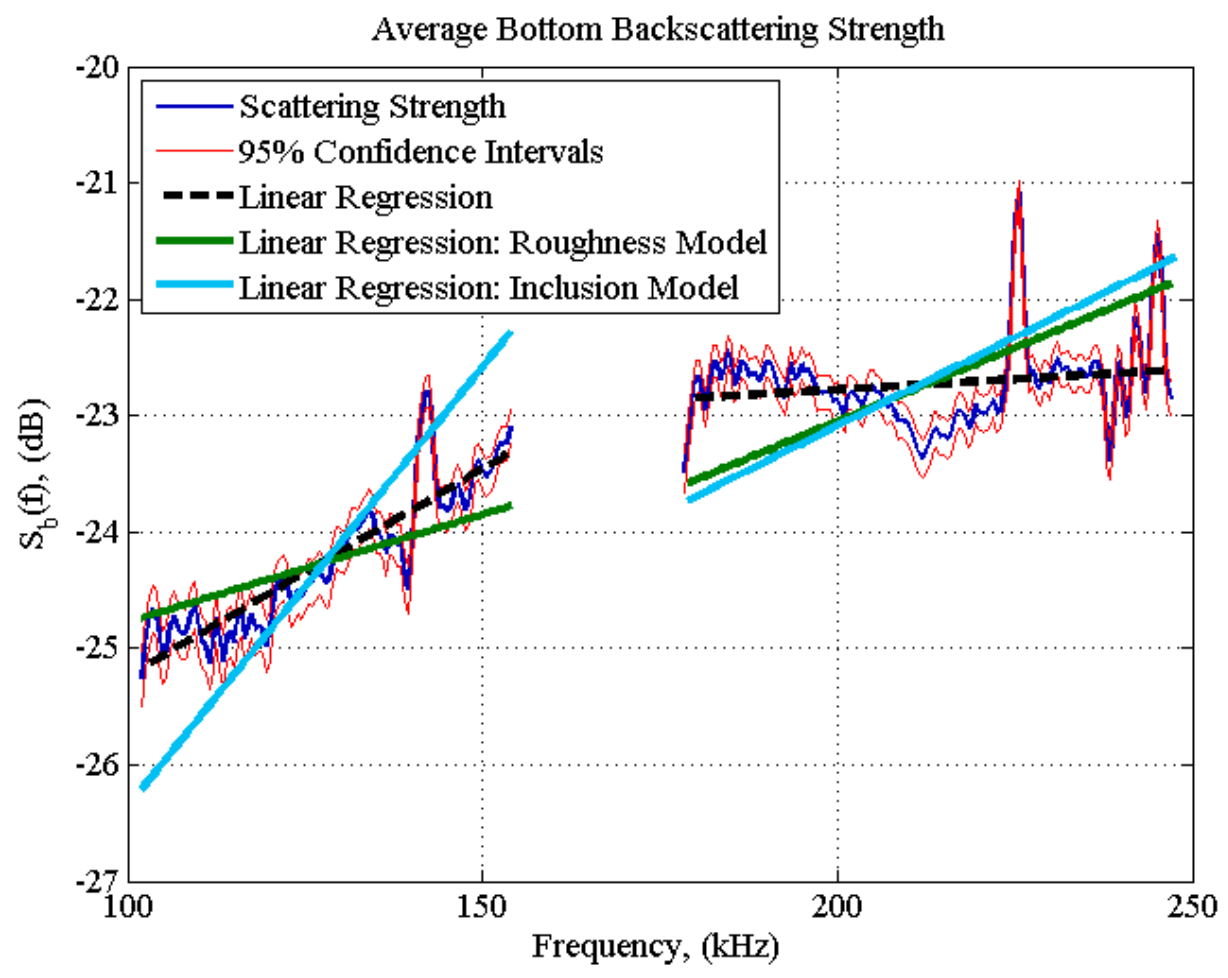

Figure 4-8. Acoustic backscatter results compared to theoretical predictions of backscatter from roughness and inclusion scattering models.

\section{Gravel Field}

Acoustic backscatter observations of the gravel field were also compared to the roughness scattering model described in Chapter 2.1. Results from 43 survey passes along a $\sim 325$ meter section (90 pings) over the gravel field were used to compare the frequency response of the acoustic data to the frequency response of the theoretical prediction of backscatter. A stability check of the backscatter results from the gravel field over the length survey period revealed that the backscatter remained relatively consistent in time varying by $1 \mathrm{~dB}-2 \mathrm{~dB}$ (Figure 4-9). 

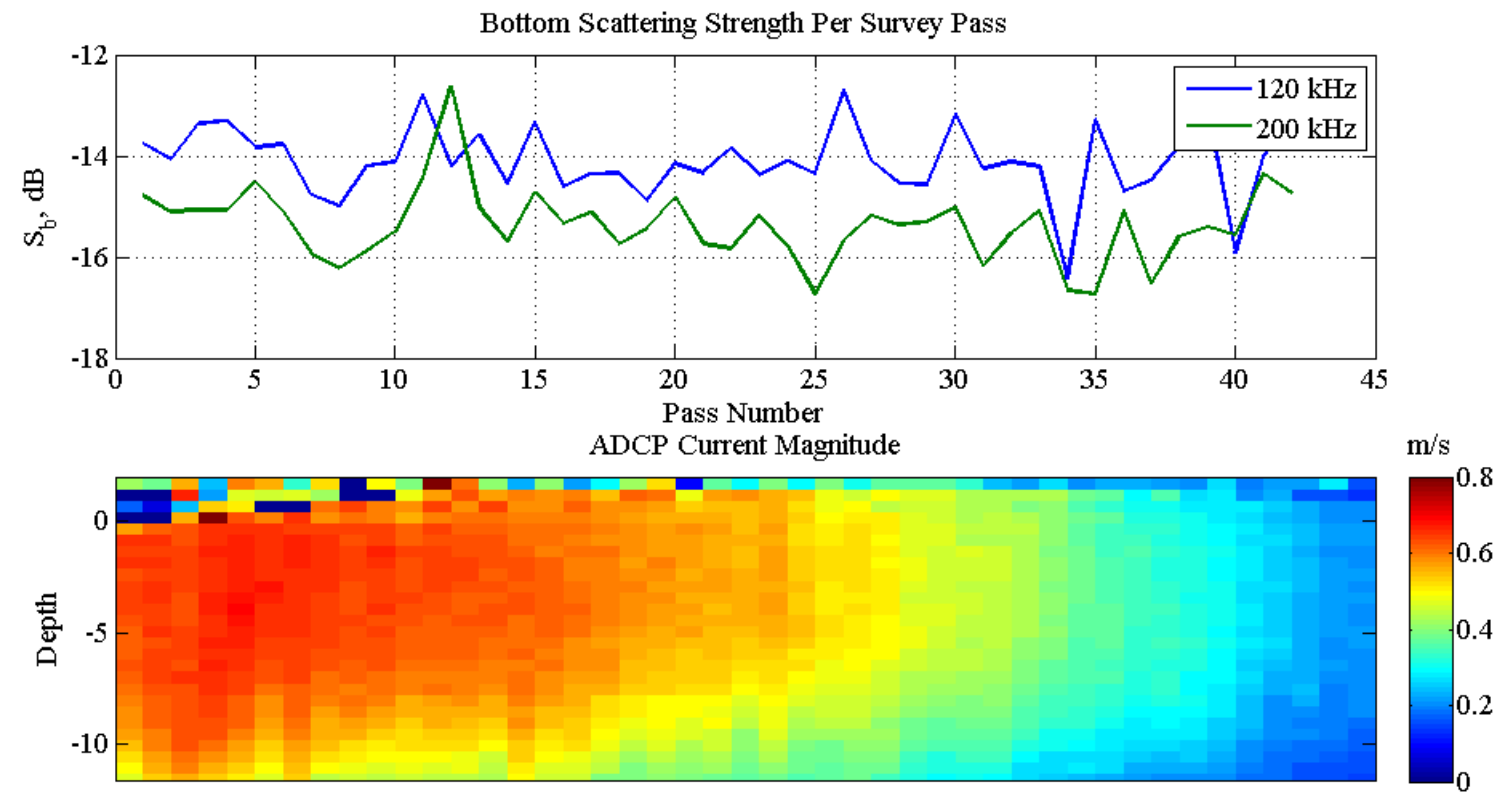

Figure 4-9. Backscatter data as a function of time over the gravel field showing stability of scattering strength throughout the duration of the survey. Magnitude of the current is shown in the bottom plot to emphasize the dynamic environment of the survey area.

Similar to that of the acoustic data, the backscatter observations from the gravel filed were averaged in time and evaluated as only a function of frequency in comparison to the roughness scattering model. 


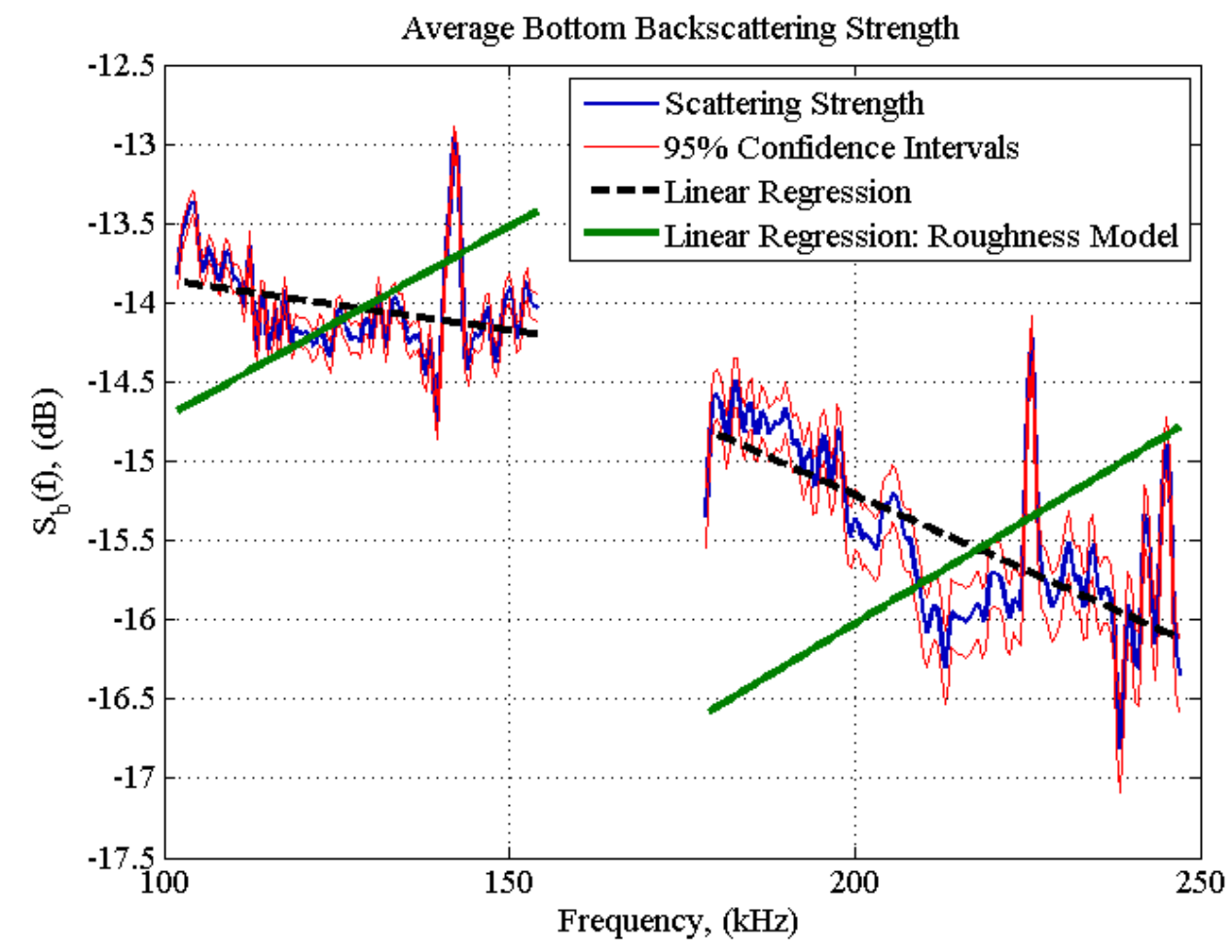

Figure 4-10. Data to model comparison of backscatter observations from the gravel field compared to the roughness scattering model.

Data to model comparisons showed that the roughness model, similar to that of the sand wave field data, was not consistent with the backscatter observations recorded over the gravel field. 


\section{CHAPTER 5}

\section{DISCUSSION}

The goal of this work was to identify the seafloor scattering mechanism that was the main contributor to calibrated acoustic backscatter observations collected in Portsmouth Harbor, New Hampshire, USA. The acoustic backscatter observations were collected over a sand wave field and a gravel field at a broad range of frequencies between $100 \mathrm{kHz}$ to $250 \mathrm{kHz}$. The study was driven by the lack of a basic understanding to what seafloor scattering mechanisms control acoustic backscatter at high frequencies, which ultimately limits our ability to accurately classify the seafloor using common acoustic remote sensing tools (e.g., shallow-water multibeam echosounders.)

Given the nature and environmental characteristics of the survey site, the two seafloor scattering mechanisms studied in correlation to the variability of acoustic backscatter observations were surficial roughness of the seabed and the presence of discrete inclusions. Both sand wave and gravel field sites were of interest in relationship to the surficial roughness of the seafloor inherent at each location. Roughness features were expected to correlate in physical size to the wavelength's of acoustic operating frequencies $(6 \mathrm{~mm}-15 \mathrm{~mm})$ being utilized in collection of acoustic backscatter. The sand wave field was also of particular interest in support of the assumption that the presence of discrete inclusions was the possible driving mechanism behind acoustic backscatter observations at this location. The sedimentary composition of the 
sand wave field was predominantly medium sand with high shell hash content providing an ideal environment under this assumption.

Independent data sets related to each seafloor scattering mechanism described, were collected and utilized as input to surficial roughness and discrete inclusion scattering models used to directly compare frequency dependent theoretical predictions of backscatter obtained from each model, to empirically observed acoustic backscatter. Analysis of the comparison between the frequency response of theoretical predictions of backscatter and empirically collected observations was implemented under the assumption that it would best indicate the underlying seafloor scattering mechanism (i.e. surficial roughness, discrete inclusions) controlling the acoustic backscatter response. Stereo imagery data sets were collected in both sand wave and gravel fields to quantitatively describe the surficial roughness of each site by way of the $2 \mathrm{D}$ roughness spectrum. Parameters derived from the roughness spectrum were used as input to the roughness scattering model to generate a theoretical prediction of backscatter unique to the sand wave and gravel fields.

A sediment sample data set was also collected within the sand wave field and subject to grain size analysis to provide the grain size distribution of the samples as input to the discrete inclusion scattering model. Estimates of acoustic parameters (i.e., sound speed and density) related to the overall sediment sample and individual grains were made as necessary inputs to the model. Estimates of such parameters effected the model in terms of its overall magnitude but not the frequency response, resulting in an accurate comparison of the theoretical prediction of backscatter to the acoustic observations as a function of frequency.

Despite the dynamic nature of the survey environment, acoustic observations from both sand wave and gravel fields were relatively stable over the duration of the survey period, varying 
slightly by only $1 \mathrm{~dB}$ to $2 \mathrm{~dB}$. Such a result was expected in the gravel field due to the large grain size distribution ( $4 \mathrm{~mm}$ to $33 \mathrm{~mm}$ ) and static behavior of the sediment. However, this result was unexpected within the sand wave field where current magnitude recorded during the survey period exceeded the threshold for incipient motion of the sediment. Movement of the sediment bottom would infer a change in the roughness spectrum, which would alter the result of backscatter observations if they were indeed controlled by surficial roughness of the seafloor. Conversely, inspection of the individual roughness spectra computed from corresponding stereo image pairs over the course of the survey period were also relatively stable in time. Sediment samples collected from the sand wave field also displayed temporally consistent behavior when compared to historically collected samples from the same area. Grain size distribution mirrored the consistency of all other data sets in time.

Upon inspection of the temporal stability of acoustic backscatter observations, roughness spectra in both gravel and sand wave fields, and sediment samples, comparison between the frequency response of acoustic backscatter observations and theoretical predictions of backscatter was made. Roughness spectra, and acoustic backscatter observations from each site were averaged in time and analyzed solely as a function of frequency. Results of time averaged acoustic backscatter overlaid by frequency dependent theoretical predictions of backscatter, derived from roughness and inclusion scattering models, is shown in Figure 5-1 for data collected over the sand wave field. 


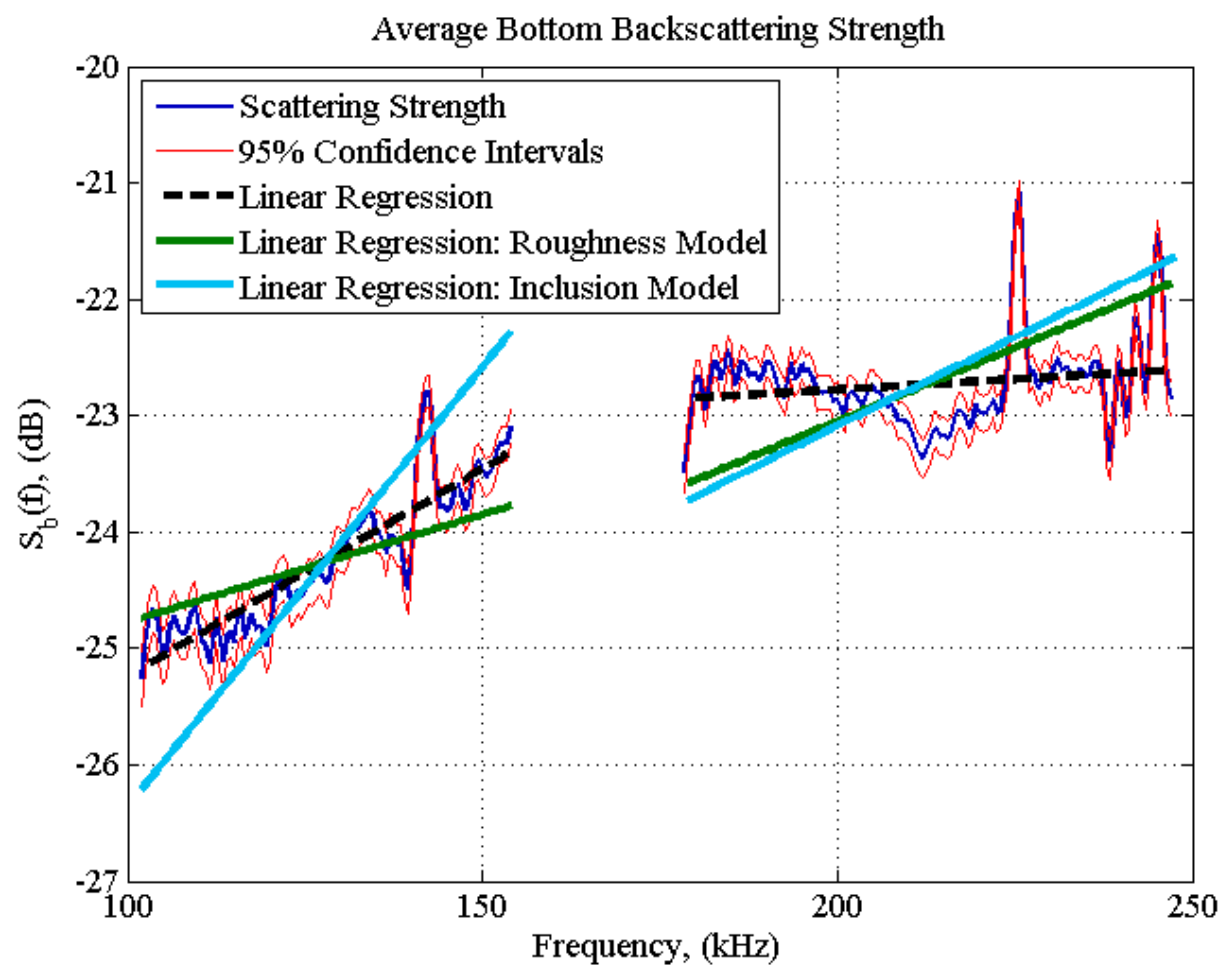

Figure 5-1. Acoustic backscatter results compared to theoretical predictions of backscatter from roughness and inclusion scattering models.

Comparison of the theoretical predictions of backscatter to empirical observations suggested that surficial roughness nor the presence of discrete inclusions were individually controlling the acoustic backscatter. Similar results were seen related to the acoustic backscatter observations collected over the gravel field in comparison to theoretical predictions from the roughness scattering model as shown in Figure 5-2. Acoustic backscatter results did not agree with the theoretical prediction of backscatter derived from the roughness scattering model. This result suggested that the acoustic backscatter response from the gravel field was not independently controlled by the surficial roughness of the seafloor environment. 


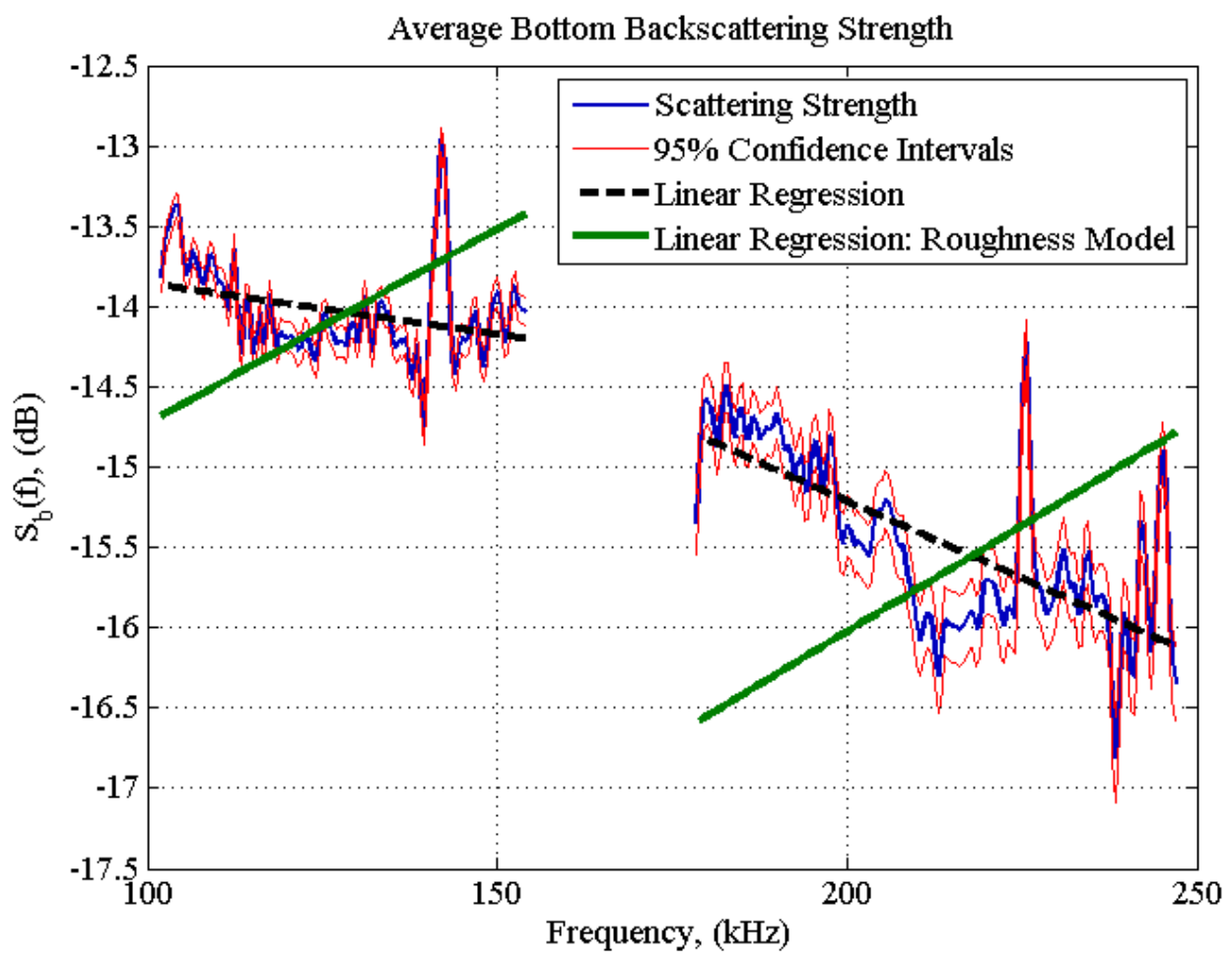

Figure 5-2. Data to model comparison of backscatter observations from the gravel field compared to the roughness scattering model.

Disagreement between acoustic backscatter results and acoustic scattering models was potentially caused by the limitation of data collection related to the stereo photography and sediment sample data sets. In terms of the sediment sample data set, the discrete inclusion scattering model was shown to be sensitive to the presence of larger grain sizes, outlined by Figure 3-29. As larger grain sizes were added to the volume size distribution, the slope of the theoretical backscatter response decreased at each frequency band. The lack of convergence of the model with the addition of larger grain sizes suggests an uncertainty of the result. If the sampling techniques utilized in the collection of the samples emitted larger grain sizes inherent to the sand wave field, the result of the theoretical prediction was potentially inaccurate. Finer sampling techniques can be implemented to reduce uncertainty of the model. 
Data to model discrepancies related to theoretical predictions of backscatter derived from stereo photography results and acoustic backscatter observations were potentially due to measurement noise inherent to the stereo photography system. The slope of individual roughness spectra depicted in Figure 3-16 relative to the sand wave field, and Figure 3-21 relative to the gravel field, were consistently noisy. Noisy behavior suggested reduced accuracy of the results, specifically over the gravel field, where no change in the surficial roughness was expected. The same assumption was true for the sand wave field at the end of the survey, when the current magnitude was reduced and the sediment became stationary. Results of the roughness spectrum should have remained consistent in areas where the surficial roughness was expected to be unchanging.

Future research aimed at improving upon the understanding of the driving seafloor scattering mechanisms behind acoustic backscatter observations should include considerations related to additional seafloor scattering mechanisms believed to effect the overall response of empirically observed acoustic backscatter. The focus of this work was related to seafloor scattering mechanisms considered inhomogeneities at the water-sediment interface. However, it has also been suggested that inhomogeneities within the sediment volume also contribute to variability in acoustic backscatter observations. Such inhomogeneities include bioturbation [Pouliquen and Lyons, 2002], gas bubbles [Anderson and Hampton, 1980], and variations in porosity [Hines, 1990]. In addition, consideration directed towards analysis of the combination of seafloor scattering mechanisms related to the variability in acoustic backscatter should also be explored. Further examination of the combination of the roughness scattering model and the discrete inclusion scattering model may potentially reveal that a combination of seafloor roughness and the presence of discrete inclusions were the controlling mechanisms of the 
acoustic backscatter response over the sand wave field. Additional analysis of multiple seafloor scattering mechanisms related to data collected over the gravel field may also reveal that the backscatter response was controlled by more than one seafloor scattering mechanism. 


\section{REFERENCES}




\section{List of References}

Anderson, Aubrey L. "Acoustics of Gas-bearing Sediments I. Background." The Journal of the Acoustical Society of America J. Acoust. Soc. Am. 67.6 (1980): 1865. Web.

Blott, Simon J., and Kenneth Pye. "GRADISTAT: A Grain Size Distribution and Statistics Package for the Analysis of Unconsolidated Sediments." Earth Surf. Process. Landforms 26 (2001): 1237-248. Print.

Bouguet, Jean-Yves. Camera Calibration Toolbox for Matlab. N.p., 2 Dec. 2013. Web. $<$ http://www.vision.caltech.edu/bouguetj/calib_doc/>.

Briggs, Kevin B., Anthony P. Lyon, Larry A. Mayer, and Michael D. Richardson. Seafloor Roughness, Sediment Grain Size, and Temporal Stability. Proc. of Underwater Acoustic Measurements: Technologies \& Results, Crete, Greece, Heraklion. Office of Naval Research, 7 Jan. 2005. Web. 23 Apr. 2015.

Brown, Craig J., and Philippe Blondel. "Developments in the Application of Multibeam Sonar Backscatter for Seafloor Habitat Mapping." Applied Acoustics 70.10 (2009): 1242-247. Web.

Burdic, William S. Underwater Acoustic System Analysis. Englewood Cliffs, NJ: Prentice Hall, 1991. Print.

Felzenburg, Janice A. "Detecting Bedform Migration from High-resolution Multibeam Bathymetry in Portsmouth Harbor, New Hampshire, USA." Thesis. University of New Hampshire, 2009. Print.

Folk, R. L., and W. C. Ward. "Brazos River Bar [Texas]; a Study in the Significance of Grain Size Parameters." Journal of Sedimentary Research 27.1 (1957): 3-26. Web. 14 Jan. 2015.

Folk, Robert L. Petrology of Sedimentary Rocks. Austin, TX: Hemphill Pub., 1980. Print.

Foote, Kenneth G., Hans Petter Knudsen, Gudmund Vestnes, D. N. MacLennan, and E. J. Simmonds. "Technical Report: "Calibration of Acoustic Instruments for Fish Density Estimation: A Practical Guide'." The Journal of the Acoustical Society of America J. Acoust. Soc. Am. 83.2 (1988): 831. Web. 15 Feb. 2015.

Hamilton, Edwin L. "Geoacoustic Modeling of the Sea Floor." The Journal of the Acoustical Society of America J. Acoust. Soc. Am. 68.5 (1980): 1313. Web. 20 Mar. 2015.

Harris, F.j. "On the Use of Windows for Harmonic Analysis with the Discrete Fourier Transform." Proceedings of the IEEE Proc. IEEE 66.1 (1978): 51-83. Web. 3 Mar. 2015 . 
Hines, Paul C. "Theoretical Model of Acoustic Backscatter from a Smooth Seabed." The Journal of the Acoustical Society of America J. Acoust. Soc. Am. 88.1 (1990): 324. Web. 2 Jan. 2015.

Ivakin, Anatoliy N. Scattering from Discrete Inclusions in Marine Sediments. Proc. of European Conference on Underwater Acoustics, The Netherlands, Delft. Applied Physics Laboratory, July 2004. Web. 5 May 2015.

Jackson, Darrell R., and M. D. Richardson. High-frequency Seafloor Acoustics. New York: Springer, 2007. 458-70. Print.

Jackson, Darrell R., Kevin B. Briggs, Kevin L. Williams, and Michael D. Richardson. "Tests of Models for High-frequency Seafloor Backscatter." IEEE Journal of Oceanic Engineering IEEE J. Oceanic Eng. 21.4 (1996): 458-70. Web. 10 Feb. 2015.

Lurton, Xavier. An Introduction to Underwater Acoustics: Principles and Applications. London: Springer, 2002. 102+. Print.

Lyons, Anthony P., Warren L.J Fox, Thomas Hasiotis, and Eric Pouliquen. "Characterization of the Two-dimensional Roughness of Wave-rippled Sea Floors Using Digital Photogrammetry." IEEE Journal of Oceanic Engineering IEEE J. Oceanic Eng. 27.3 (2002): 515-24. Web. 20 Feb. 2015.

Mckinney, C. M., and C. D. Anderson. "Measurements of Backscattering of Sound from the Ocean Bottom." The Journal of the Acoustical Society of America J. Acoust. Soc. Am. 36.1 (1964): 158. Web.

Pouliquen, Eric, and Anthony P. Lyons. "Backscattering from Bioturbated Sediments at Very High Frequency." IEEE Journal of Oceanic Engineering IEEE J. Oceanic Eng. 27.3 (2002): 388-402. Web. 5 Apr. 2015.

Richardson, M.d., K.b. Briggs, L.d. Bibee, P.a. Jumars, W.b. Sawyer, D.b. Albert, R.h. Bennett, T.k. Berger, M.j. Buckingham, N.p. Chotiros, P.h. Dahl, N.t. Dewitt, P. Fleischer, R. Flood, C.f. Greenlaw, D.v. Holliday, M.h. Hulbert, M.p. Hutnak, P.d. Jackson, J.s. Jaffe, H.p. Johnson, D.l. Lavoie, A.p. Lyons, C.s. Martens, D.e. Mcgehee, K.d. Moore, T.h. Orsi, J.n. Piper, R.i. Ray, A.h. Reed, R.f.l. Self, J.l. Schmidt, S.g. Schock, F. Simonet, R.d. Stoll, D. Tang, D.e. Thistle, E.i. Thorsos, D.j. Walter, and R.a. Wheatcroft. "Overview of SAX99: Environmental Considerations." IEEE Journal of Oceanic Engineering IEEE J. Oceanic Eng. 26.1 (2001): 26-53. Web. 20 Apr. 2015.

Rothermel, Mathias, Konrad Wenzel, Dieter Fritsch, and Norbert Haala. SURE:

Photogrammetric Surface Reconstruction from Imagery. Proc. of LC3D Workshop, Germany, Berlin. University of Stuttgart, Nov. 2012. Web. 2 Dec. 2014.

Sherwood, Christopher R., Jessica R. Lacy, and George Voulgaris. "Shear Velocity Estimates on the Inner Shelf off Grays Harbor, Washington, USA." Continental Shelf Research 26.17-18 (2006): 1995-2018. Web. 8 Feb. 2015. 
Stanic, S., Kevin B. Briggs, P. Fleischer, W. B. Sawyer, and R. I. Ray. "High-frequency Acoustic Backscattering from a Coarse Shell Ocean Bottom." The Journal of the Acoustical Society of America J. Acoust. Soc. Am. 85.1 (1989): 125-36. Web. 13 Jan. 2015.

Stanic, S., R.r. Goodman, K.b. Briggs, N.p. Chotiros, and E.t. Kennedy. "Shallow-water Bottom Reverberation Measurements." IEEE Journal of Oceanic Engineering IEEE J. Oceanic Eng. 23.3 (1998): 203-10. Web. 15 Feb. 2015.

Stuart, Elaine. "What Is Multibeam Backscatter?" NOAA Ocean Explorer Podcast RSS. National Oceanic and Atmospheric Administration, Aug. 2012. Web. 23 July 2015.

Thorsos, Eric I. "SAX99: An Experiment in High-frequency Sediment Acoustics." The Journal of the Acoustical Society of America J. Acoust. Soc. Am. 108.5 (2000): 2510. Web. 8 Feb. 2015.

Ward, Larry G. and Zachary S. McAvoy. "Bottom Sediment Characteristics." Center for Coastal and Ocean Mapping/Joint Hydrographic Center, University of New Hampshire, August 2014. Document.

"Water Levels - NOAA Tides \& Currents." Water Levels - NOAA Tides \& Currents. NOAA / National Ocean Service, n.d. Web. 20 July 2015.

Weber, Thomas C. "Acoustic Seabed Interaction Theory." Seabed Acoustic Backscatter. 9 Jan. 2014. Lecture.

Weber, Thomas C. "Observations of Backscatter from Sand and Gravel Seafloors Between 170 250 KHz." The Journal of the Acoustical Society of America J. Acoust. Soc. Am. (2015): 1. Print.

Williams, K.1., D.r. Jackson, Dajun Tang, K.b. Briggs, and E.i. Thorsos. "Acoustic Backscattering From a Sand and a Sand/Mud Environment: Experiments and Data/Model Comparisons." IEEE Journal of Oceanic Engineering IEEE J. Oceanic Eng. 34.4 (2009): 388-98. Web. 9 Feb. 2015.

Williams, K.1., D.r. Jackson, E.i. Thorsos, Dajun Tang, and K.b. Briggs. "Acoustic Backscattering Experiments in a Well Characterized Sand Sediment: Data/model Comparisons Using Sediment Fluid and Biot Models." IEEE Journal of Oceanic Engineering IEEE J. Oceanic Eng. 27.3 (2002): 376-87. Web. 3 Apr. 2015. 
APPENDIX 
HackHD Stereo Camera System User's Manual 


\section{INTRODUCTION:}

The HackHD Stereo Camera System (HSCS) consists of three HackHD cameras and two LED light sources. Two cameras are used as part of the stereo camera system and one is used as an observation camera. The system is mounted to a sea tripod when used for deployment with a fixed focal length of 1 meter; $\sim \operatorname{lm} \times 1 \mathrm{~m}$ field of view (FOV) in water. The baseline of the stereo cameras is $40 \mathrm{~cm}$ and each is tilted inward at an angle of $10^{\circ}$ from the horizontal. All three cameras are programmed with an Arduino Uno controller to record images simultaneously.

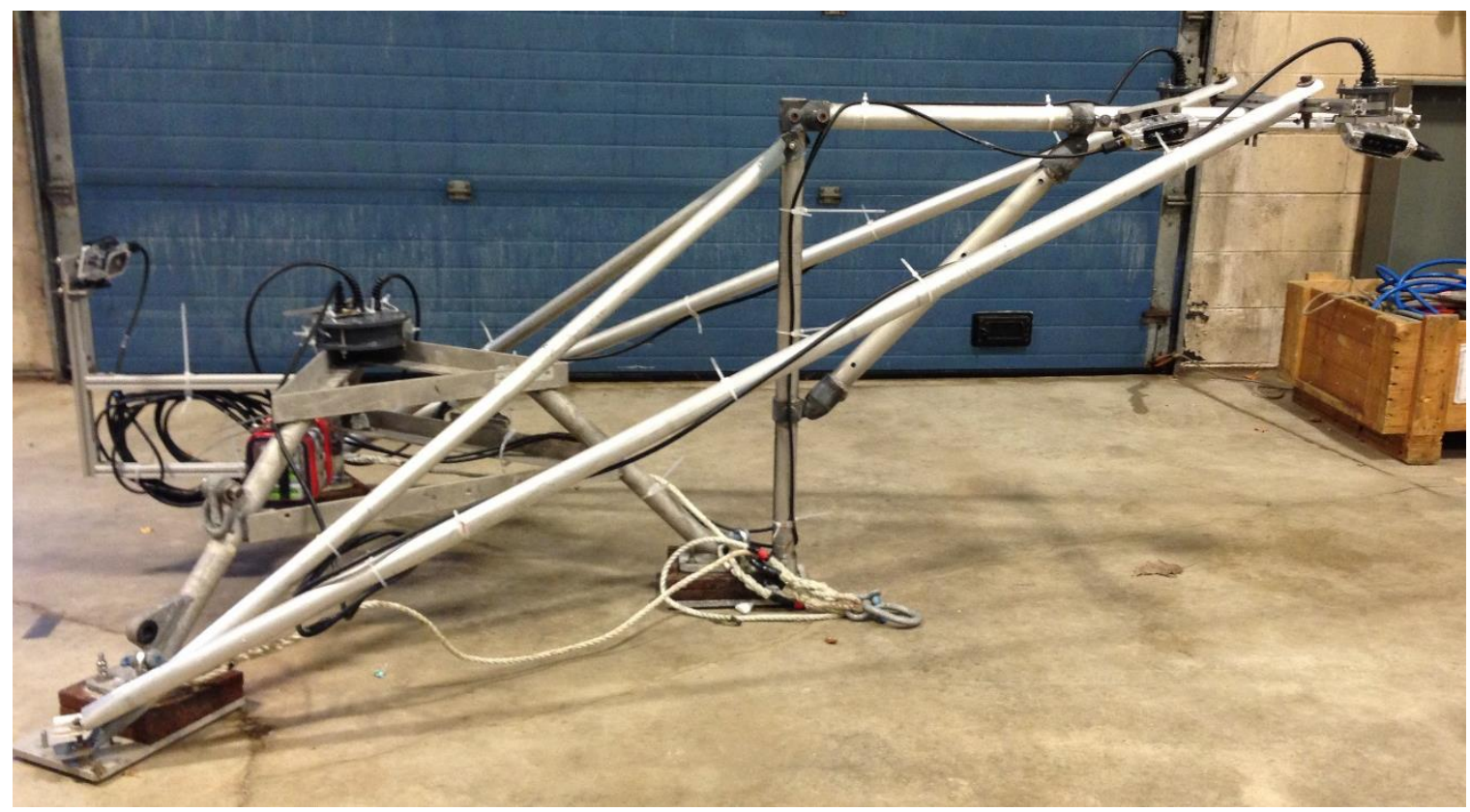




\section{SYSTEM OVERVIEW:}

\section{Camera Specifications:}

Resolution: 1080P HD Video / 5MP or 9MP Still Photo

Frame Rate: 30 FPS (frames per second) Video

Coding: H.264

Aspect Ratio: 16:9

File Format: AVI Video / JPG Photo

Storage: External microSD or microSDXC card (Supports SDHC cards with adapter)

Lens: Interchangeable M12 Lens. Includes 2.5mm (EFL), F2.8, 160 degree (diagonal) wide angle lens

Control Input: Single contact monetarily switch

Video Output: Composite video 480P resolution

Status Indicator Output: Single color LED driver

Dimensions: $65 \mathrm{~mm}$ x 40mm x 25mm LxWxH

Power Supply: External 3.7V, 1100mAH minimum. 5V safe.

Low battery procedure: $3.6 \mathrm{~V}$ indicates low battery by faster LED blinking, $3.4 \mathrm{~V}$ stops

recording/turns off

Power Output: 3.7V DC, 500mAH

Working Temperature: $-10 \operatorname{degC}$ to $+45 \operatorname{degC}$

Storage Temperature: $-20 \mathrm{degC}$ to $+70 \mathrm{degC}$

Sensor: 
Manufacture: Omnivision

Sensor Size Format: 1/2.3 inch

Number of Pixels : 9 mega pixels

Pixel size: $1.75 \mathrm{um}$ x $1.75 \mathrm{um}$

Sensitivity: 960mV/lux-sec

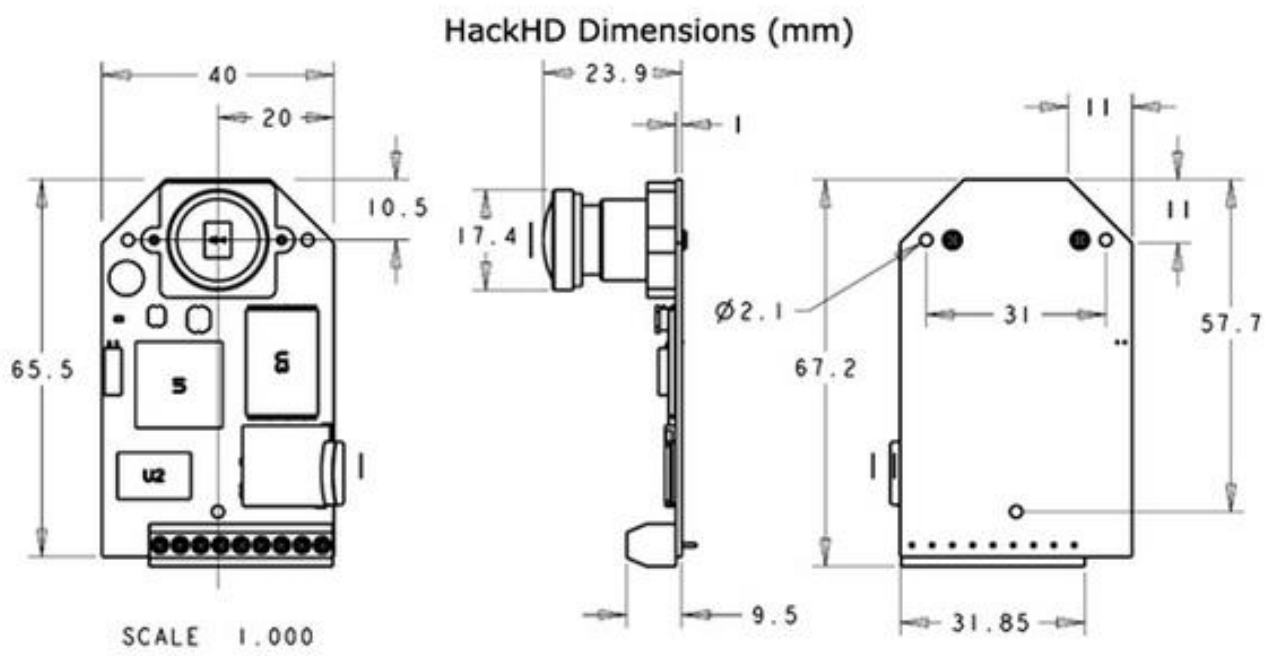

Figure 3. HackHD camera dimensions.

\section{Stereo Camera Lens Specifications:}

4.0mm $80^{\circ} \mathrm{F} 2.0$ Lens HACKHD-LENS-018

Focal Length: $4.0 \mathrm{~mm}$

Back Focal Length: 6.26mm

Format: 1/3", 1/4"

Aperture: F2.0

Angle of View: $80^{\circ}, 64^{\circ}$

Dimension: $14.0 \times 15.5 \mathrm{~mm}$

Weight: $5.5 \mathrm{~g}$

Lens Construction: $4 \_4$ 


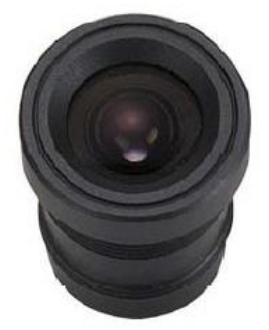

\section{LED Light Specifications:}

Figure 4. Lens used for each stereo camera.

White 30HP-LED Tectangle G4 Lamp

Dimensions: $4.22 \mathrm{~cm}(1.66 ")$ x $3.164 \mathrm{~cm}(1.25 ")$ x $3.164 \mathrm{~cm}(1.25 ")$

\begin{tabular}{|c|c|c|c|}
\hline Base & G4 & Beam Angle & 120 degree \\
\hline Comparable Wattage & 20 25 Watts & Current Draw @ Operating Voltage & $115 \mathrm{~mA}$ \\
\hline Dimmable & Yes & Efficacy & $86 \mathrm{~lm} / \mathrm{w}$ \\
\hline IP Rating & Non-Weatherproof & LED Quantity & 30 LEDs \\
\hline LED Type & 3528 SMD & Lens & Clear \\
\hline Lifetime & 50000 Hours & Lumen & 120 Lumen \\
\hline Lumen Per LED & 4 & MCD Per LED & 1300 \\
\hline Millicandela & $39000 \mathrm{mcd}$ & Operating Temperature & $-30 \sim+80{ }^{\circ} \mathrm{C}$ \\
\hline Oversize & Yes & Polarity Sensitive & No \\
\hline $\begin{array}{l}\text { Standards And } \\
\text { Certifications }\end{array}$ & $\begin{array}{l}\text { CE/FCC/ROHS } \\
\text { Compliant }\end{array}$ & Storage Temperature & $-40 \sim+90{ }^{\circ} \mathrm{C}$ \\
\hline $\begin{array}{l}\text { Total Power } \\
\text { Consumption }\end{array}$ & 1.4 Watts & Type & Bi-Pin \\
\hline $\begin{array}{l}\text { Operating Voltage } \\
\text { Range }\end{array}$ & $12 \mathrm{~V} \mathrm{AC/DC}$ & & \\
\hline
\end{tabular}




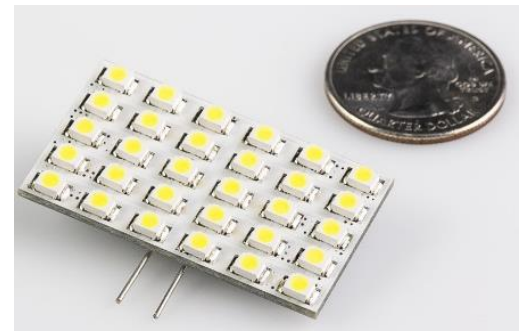

\section{Power Consumption:}

Figure 5. LED lights use with HSCS.

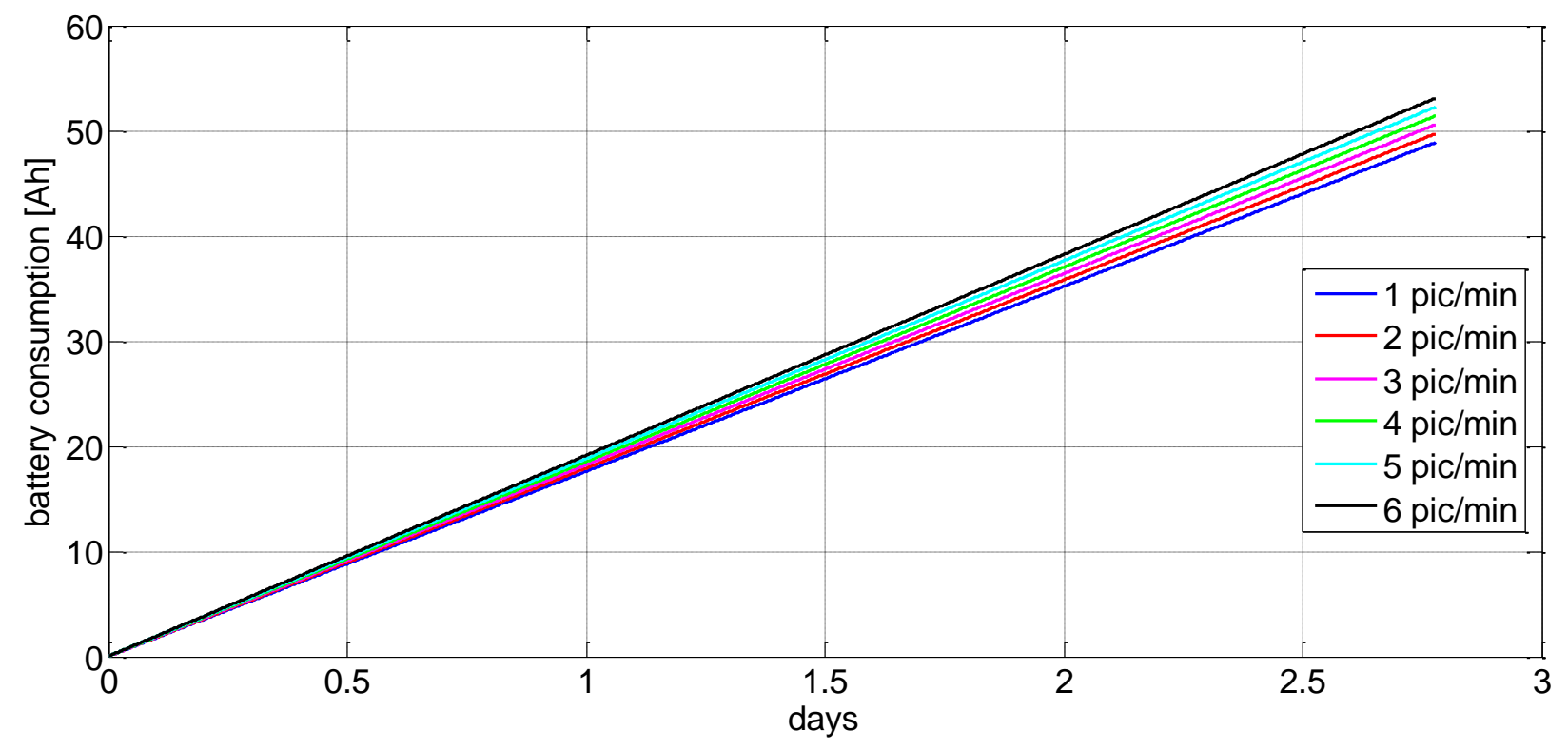

Figure 6. Power Consumption of HSCS.

\section{Number of Pictures in SD Card ( 7200 max.):}




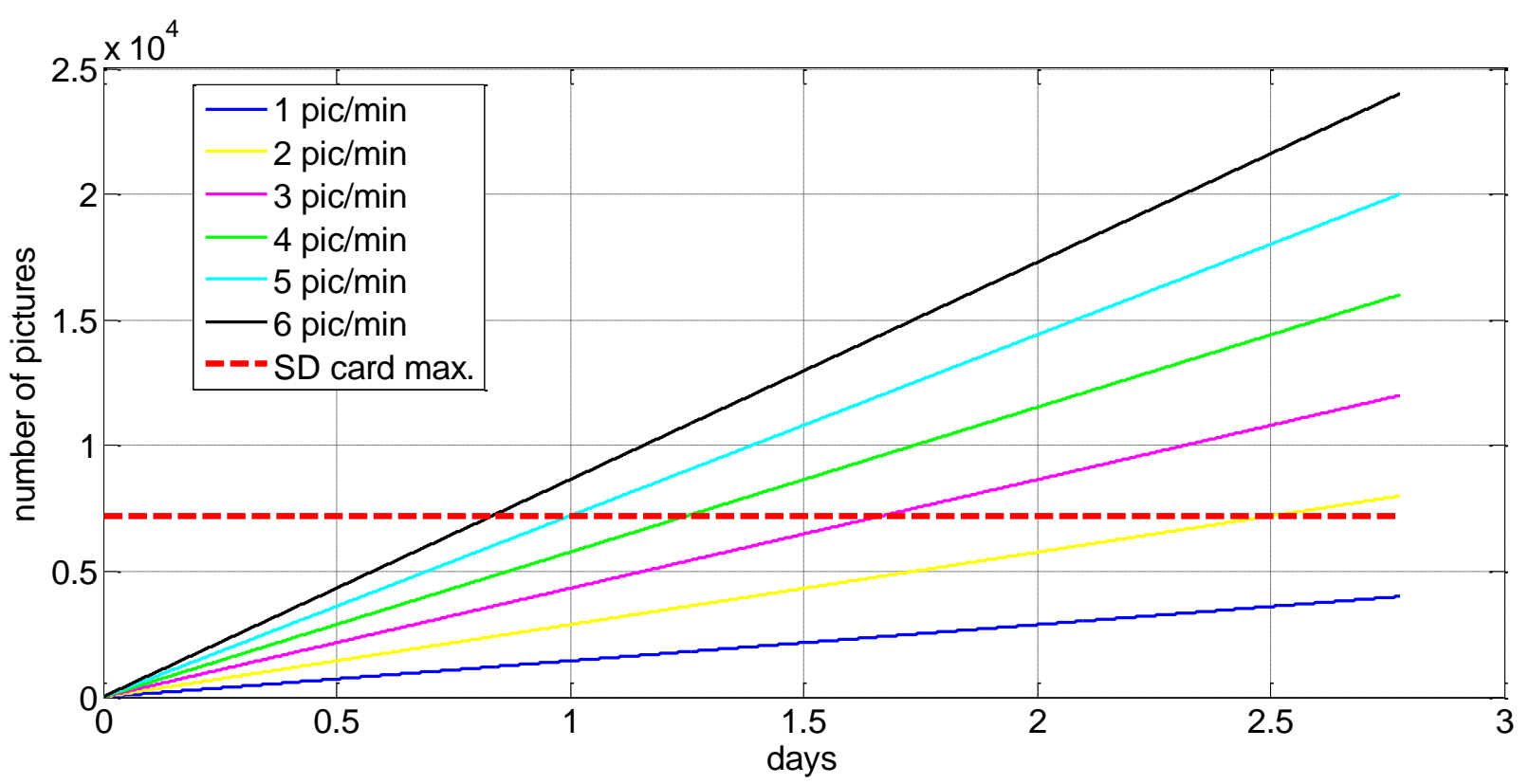

Figure 7. SD Card memory estimation for HSCS. 


\section{POST PROCESSING METHODS:}

\section{Calibration Process:}

*The calibration process can occur before or after imagery data collection but the cameras must remain in the same orientation during each process; calibration and data collection.

1. Set stereo cameras in final resting position. The back of the stereo camera's case should be parallel with angle aluminum mounting hardware. Stereo cameras must remain in the same position for both the calibration process and data collection.

2. Take 10-12 pictures of $1 m \times 1 m$ black and white checkerboard calibration target.

- Stereo calibration images should be taken in the same medium as intended data collection medium. To obtain images, place the calibration target underneath the HSCS with the numbered side up. (This becomes important in calibration post processing) Tilt the target in different orientations for each image to obtain suitable calibration images.

3. Load images onto PC into separate folders for left and right calibration images.

- Ex. Left Calibration Images | Right Calibration Images

4. Run stereo calibration software that can be downloaded here: TOOLBOX_calib http://www.vision.caltech.edu/bouguetj/calib_doc/ *It is important to calibrate each camera's images separately but to remain consistent when selecting grid corners on image pairs.

- Start two Matlab windows and open calib_gui.m from the TOOLBOX_calib library in each window.

- Each window's working directory should be the location of either the left or right calibration images. When you run calib_gui.m, add its path to the Matlab path instead of changing directories.

*THE FOLLOWING STEPS PERTAIN TO EACH MATLAB WINDOW AND

\section{SHOULD BE DONE SIMULTANEOUSLY!!!}

- After running calib_gui.m a GUI appears on the screen:

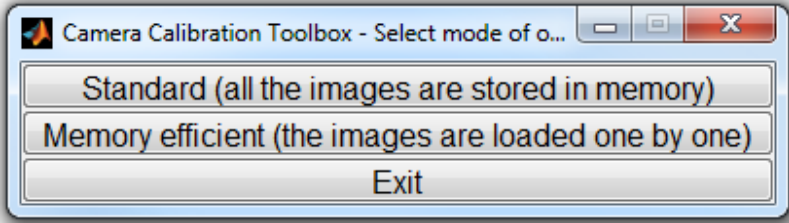


- Select 'Standard (all images are stored in memory)'

- A second GUI will appear on the screen:

\begin{tabular}{||c|c|c|c|c|}
\hline \multicolumn{1}{|l|}{ Camera Calibration Toolbox - Standard Version } & & \\
\hline Image names & Read images & Extract grid corners & Calibration \\
\hline Show Extrinsic & Reproject on images & Analyse error & Recomp. corners \\
\hline Add/Suppress images & Save & Load & Exit \\
\hline Comp. Extrinsic & Undistort image & Export calib data & Show calib results \\
\hline
\end{tabular}

- Select the option 'Read images' and follow the steps that appear in the Matlab Command Window. When complete a figure containing all calibration images should appear:

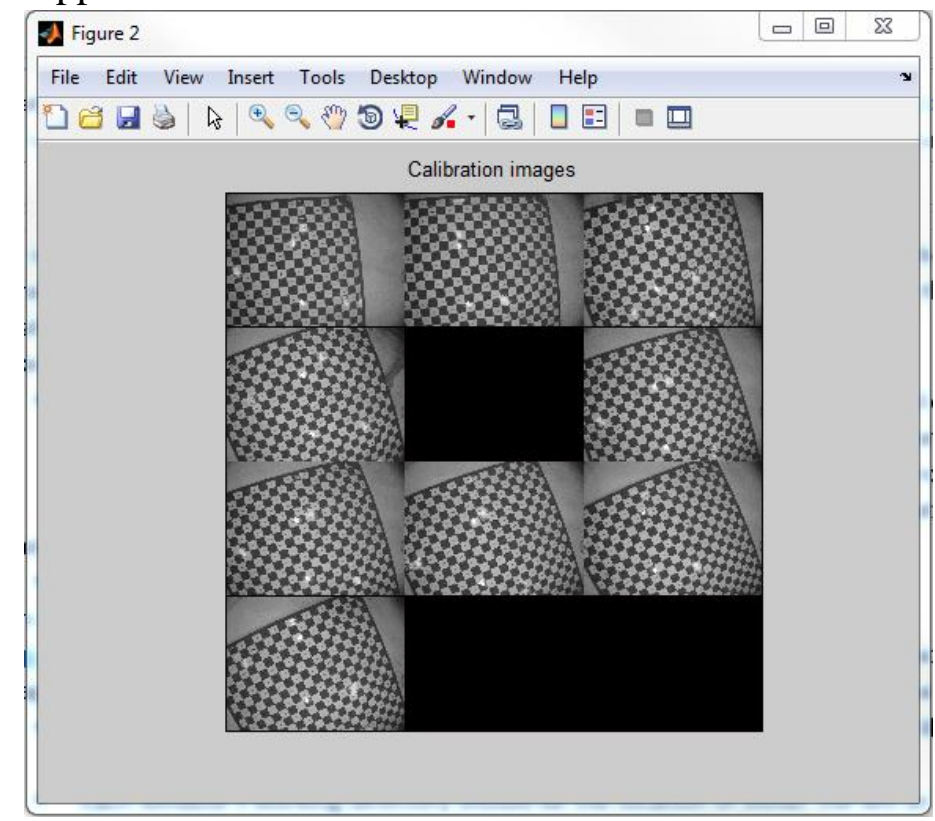

*If a black space appears, that is because Matlab reads in the images chronologically based on their file name. If one is missing, it replaces it with a black square and will be ignored for the remainder of the process.

- Select the option 'Extract grid corners' from the GUI. Follow the instructions in the Matlab Command Window.

o wintx and winty should be set to 38 for this system.

○ Enter ' 1 ' to manually enter the number of squares.

- SELECT THE SAME FOUR EXTREME CORNERS FOR BOTH LEFT AND RIGHT IMAGES.

- Enter number of squares in $X$ and $Y$ directions. 
- Enter $50 \mathrm{~mm}$ for square size in $d X$ and $d Y$ directions.

- Enter ' $y$ ' to enter in initial guess for radial distortion. For this system, start with 0.25 and work from there. The figure title will be 'The red crosses should be close to the image corners'. Entering an initial guess should achieve this.
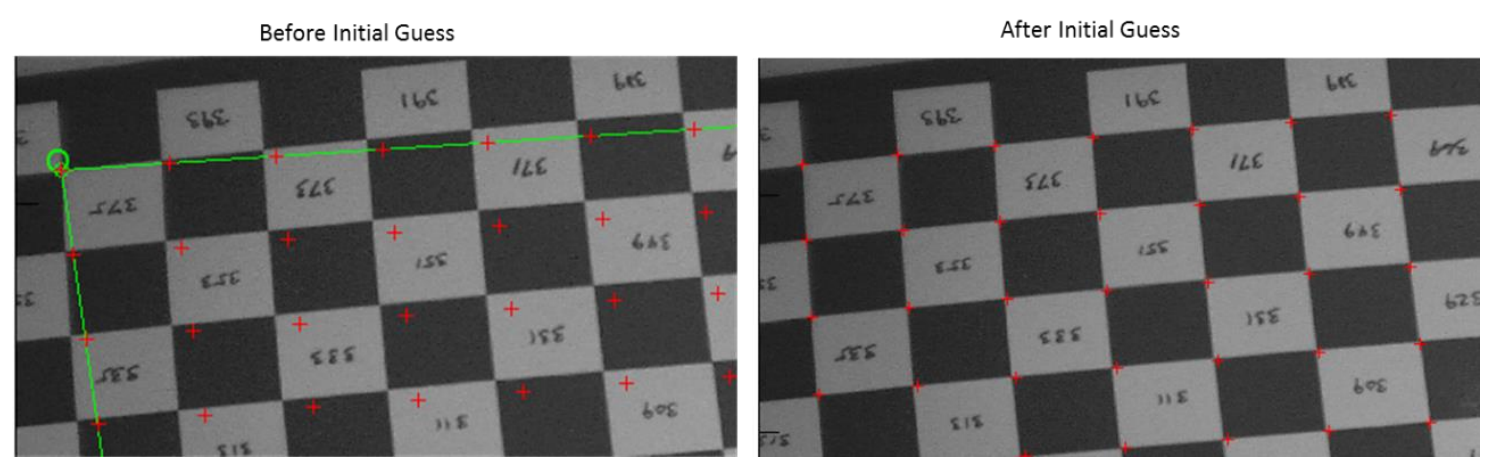

- Resulting red cross hairs should all be on intersecting black and white squares.

- When all images have been process, a file called calib_data.mat will be created in the directory where the images are stored.

- Return to the GUI and select the 'Calibration' option (calibration will occur and you should see something show up in the Matlab Command Window) then the 'Save' option, which will create two files: Calib_Results.m and Calib_Results.mat

- Rename each Calib_Results.mat file,Calib_Results_left.mat and Calib_Results_right.mat in each respective folder.

5. Close one of the Matlab windows and open stereo.gui.m in the remaining window. Run the script. Be sure to add the path of each Calib_Results_* before selecting on options on the GUI that appears on the screen.

- Select 'Load left and right calibration files' from the gui. The Matlab Command Window will suggest file names for left and right calibration result files which were renamed in the last step.

- Load the two files and then select 'Run stereo calibration' from the GUI.

- Copy and paste results that appear in the command window to a text file and save for later use. Save with the file extension *.slcp

- Select 'Exit' from GUI.

6. The calibration process is now complete. 


\section{Image Correction Process:}

The image correction process in done using the calibration file produced in the previous section in conjunction with the program LensCorrect.exe. (Yuri Rzhanov)

1. Open the program LensCorrect.exe.

2. On the top tab bar, select the option 'Folder'

3. Browse your directory for the folder that contains the images you wish to correct.

4. Drag and drop the *.slcp file created in the previous section into the LensCorrect.exe program.

5. A window will pop up asking which set of parameters to use for the correction. Select the option that correlates to the folder of images selected in Step 3; left or right.

6. Click 'Correct'. Depending on the number of images you wish to correct, this may take a while. The program will save the new corrected images in the same folder with an added $-\mathrm{c}$ at the end of the file name (e.g. Original filename: Picture 1.JPG |Corrected filename: Picture1-c.JPG

7. Close the LensCorrect.exe program. 


\section{Stereo Imagery 3D Reconstruction:}

This section will provide insight into creating 3D images and 3D point files from stereo image pairs. First it will discuss creating *.las files from stereo image pairs, which contain x,y,z values, RGB values, etc. (see latest LAS Specification document for all data within *.las file. It will then go over creating 3D images from the *.las and how to extract the data of interest from *.las files to *.txt files.

Create *.las files from stereo image pairs using SURE.exe: (Yuri Rzhanov/ $\mathrm{Han} \mathrm{Hu}$ )

This process will be an outline for preparing a pair of stereo images. When working with numerous stereo image pairs, writing a windows batch file to automate the process is highly suggested.

1. Using both the left and right camera calibration parameters (*.slcp file), call the function prdori.m (by $\mathrm{Han} \mathrm{Hu}$ )

a. Input parameters: $\mathrm{fx} 1$ - left camera focal length $\mathrm{x}$

$$
\begin{aligned}
& \text { fy1 - left camera focal length y } \\
& \text { px1 - left camera principal point } x \\
& \text { py1 - left camera principal point y } \\
& \text { fx2 - right camera focal length } x \\
& \text { fy2 - right camera focal length y } \\
& \text { px2 - right camera principal point } x \\
& \text { py2 - right camera principal point } y \\
& \text { om - rotation column vector } \\
& t \text { - translation column vector }
\end{aligned}
$$

2. Two $3 \times 3$ projection matrices will appear in the Matlab Command Window. The first will be the left camera projection parameters and the second will be the right 
camera projection parameters. Copy each matrix into a separate text file and name them left.txt and right.txt, respectively.

3. Create two folders called image and ori in a new directory.

4. Put a pair of stereo images into the image folder. Make sure they are corrected images $\left(*_{-}\right.$.JPG)

5. Copy and paste left.txt and right.txt into the ori folder and rename them the same name as their corresponding image.

6. In a Windows Explorer window open in the directory of the image and ori folders, hold shift and right click to select 'Open command window here'

7. Run SURE.exe in Windows Command Line:

Sure.exe -img ./image -ori ./ori

Note: To run SURE from any directory you want, you need to add the bin directory to your system path.

8. A folder SURE is generated and the 3D point clouds are saved in SURE/3D_Points with *.las file extensions. 


\section{Extracting $X Y Z$ and RGB values from *.las using CloudCompare.exe:}

CloudCompare.exe is a free software that can be found on the world wide web and is used to reproduce 3D imagery from *.las files. For this purpose, it will be used to convert *.las files to *.txt files containg XYZ and RGB values. This method is useful for a small amount of images.

1. Open CloudCompare.exe

2. Drag and drop *.las file into program.

3. Highlight cloud file in DB Tree. The cloud file will be named pts_originalFilename Cloud.

4. Click file and save with *.txt file extension.

5. Close CloudCompare.exe

Extracting XYZ and RGB values from *.las using $C++$ programming API LASlib:

LASlib is a $\mathrm{C}++$ programming API that can be used to read *.las files and can be

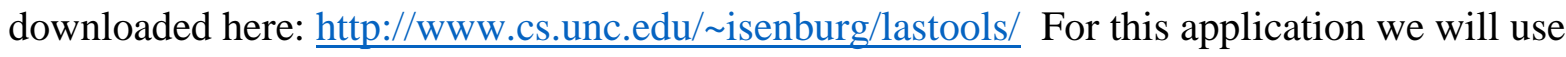
las2txt.exe.

The website also provides README files for each one of its programs which can be helpful in understanding how to extract what is needed from each *.las file.

This method is explained for a single *.las file but can be applied to a Windows batch file for batch processing of many images.

1. Open a Windows Command Line window in the same directory as las2txt.exe by holding shift and right clicking. Select the option 'Open command window here'

2. Copy and paste *.las file of interest into the directory where las2txt.exe exists. (Similar to SURE.exe, adding this directory to your system path will allow you to run las2txt.exe from any directory.)

3. On the Windows Command Line and run las2txt.exe.

last2txt -i inputFILE.las -o outputFILE.txt -parse xyzRGB

4. A text file is generated and places $x, y$,and $z$ coordinates at the $1^{\text {st }}, 2^{\text {nd }}$, and $3^{\text {rd }}$ entry and the $\mathrm{r}, \mathrm{g}$, and $\mathrm{b}$ value of the RGB color as the $4^{\text {th }}, 5^{\text {th }}$, and $6^{\text {th }}$ entry of each line. 


\section{HSCS WIRING DIAGRAM:}

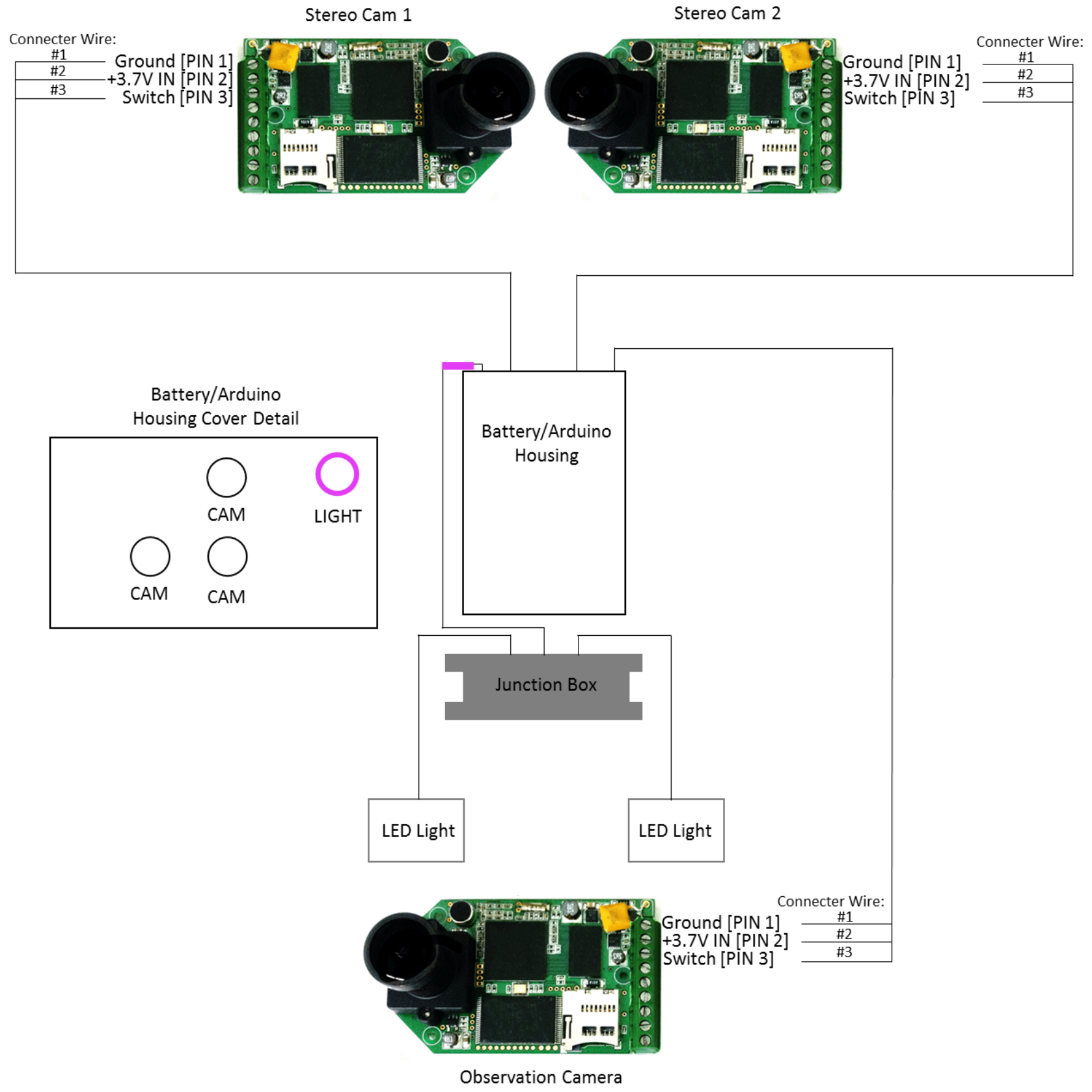

Figure 8. HSCS wiring diagram. 
PICTURE DETAIL:

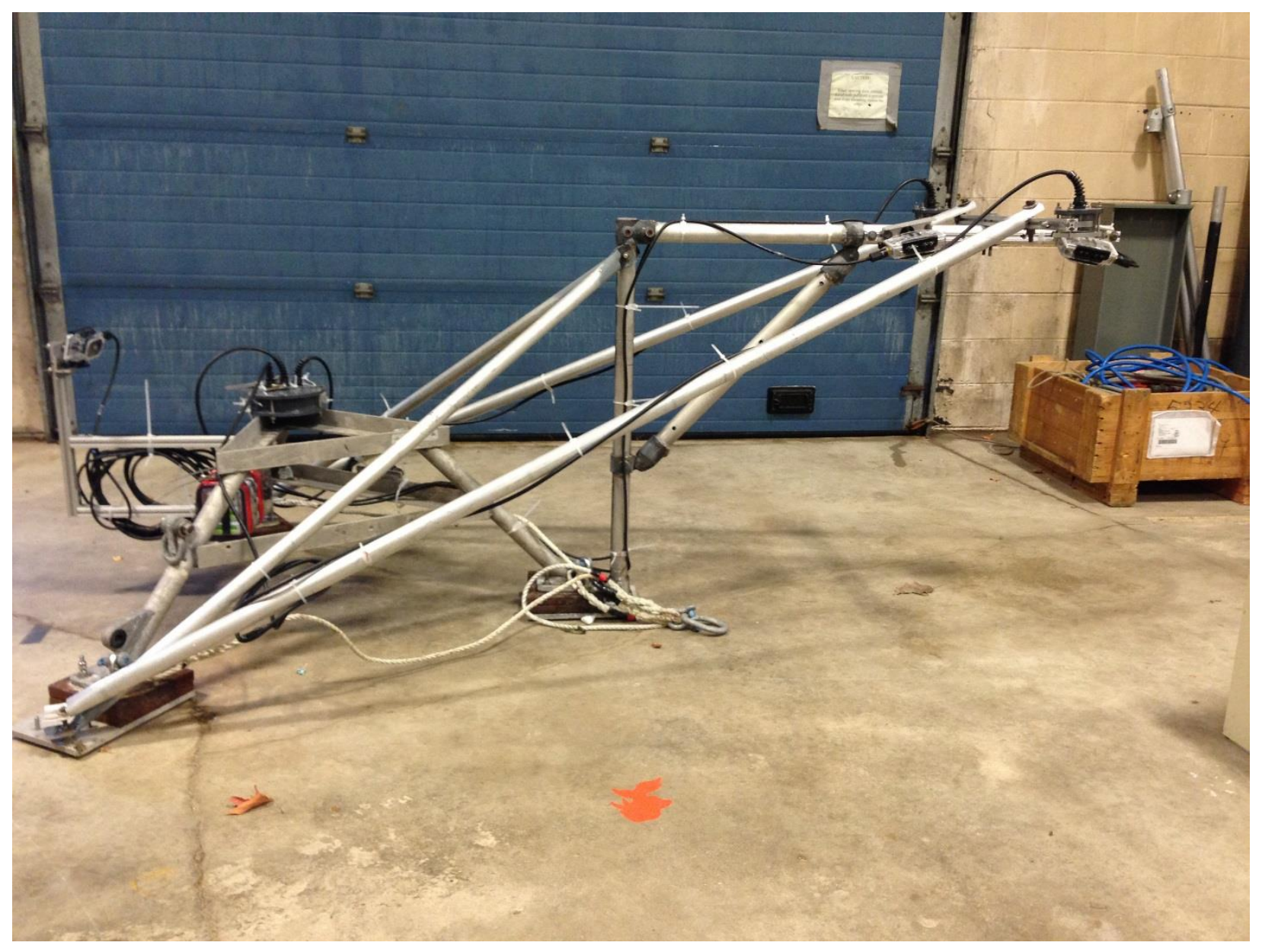



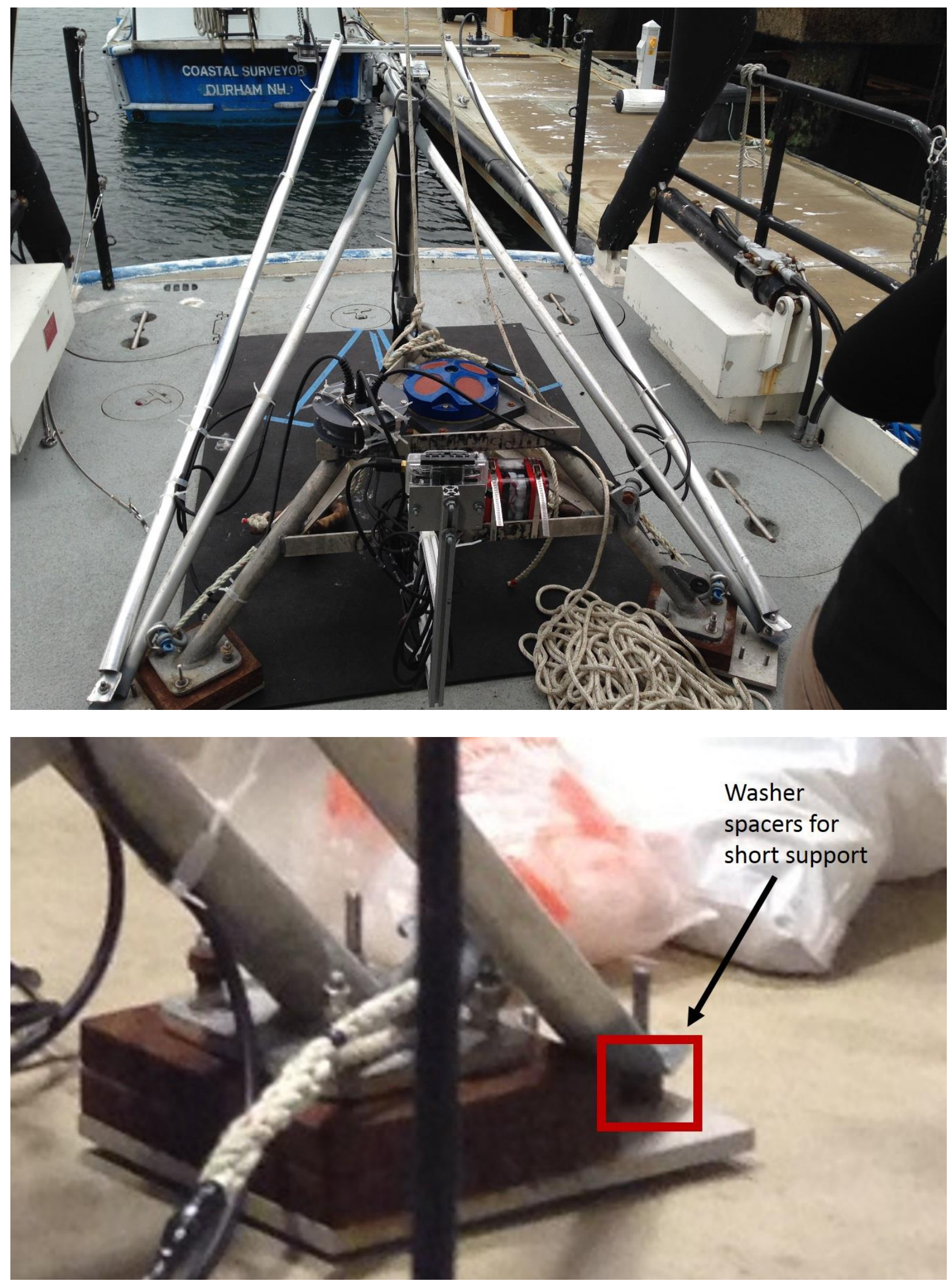


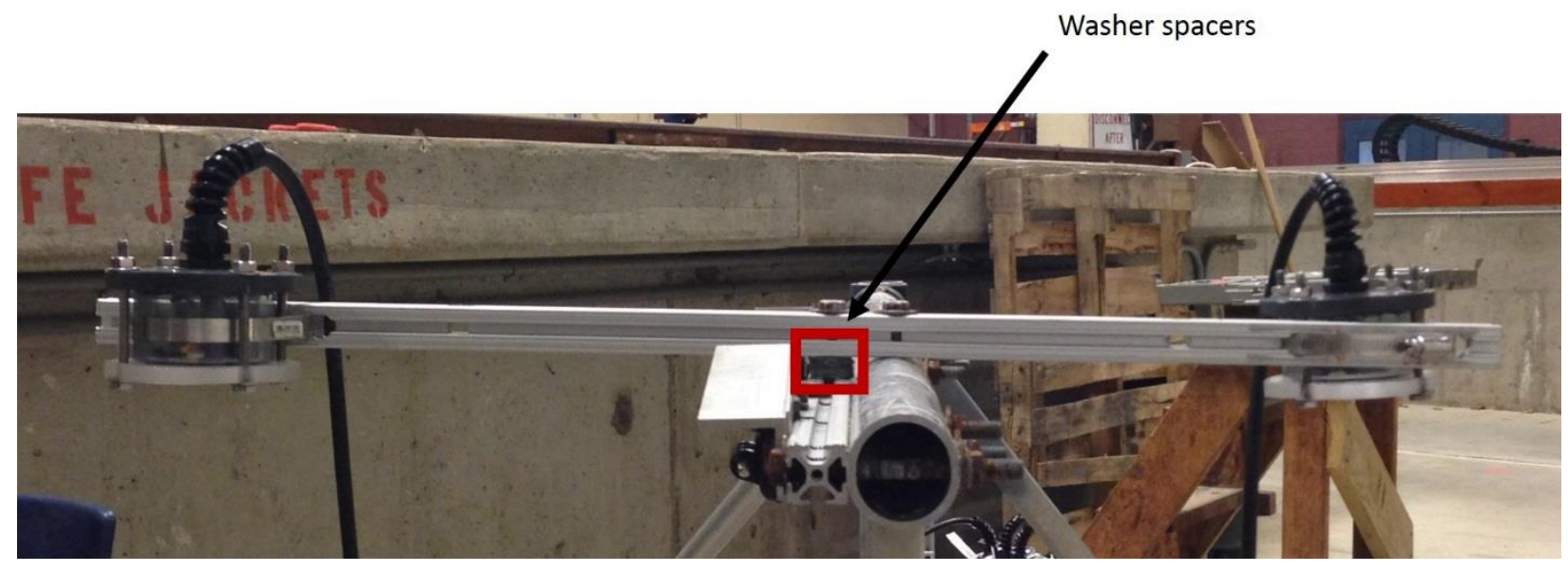

